\title{
Studying Amphiphilic Self-assembly with Soft Coarse-Grained Models
}

\author{
Marcus Müller
}

Received: 18 April 2011 / Accepted: 4 August 2011 / Published online: 2 September 2011

(C) The Author(s) 2011. This article is published with open access at Springerlink.com

\begin{abstract}
Highly coarse-grained models for investigating the self-assembly of lipids and copolymer materials are discussed. Soft interactions between segments that represent many atoms naturally arise in the course of systematic coarse-graining, and they are necessary for modeling fluctuation effects whose strengths is dictated by a large invariant degree of polymerization. The soft non-bonded interactions of the coarse-grained models are related to the excess free-energy functional of an equivalent field-theoretic description. The connection between the particle-based model and the field-theoretic description helps to identify the physical significance of the model interactions. Non-bonded interactions, which describe the complex phase behavior of compressible mixtures or include local fluid-like packing effects of the coarse-grained segments, can be systematically constructed based on liquid-state theory or classical density functional theory. Details of the computational implementation and limitations of soft coarse-grained models are discussed. Two computational techniques-field-theoretic force-matching and umbrella sampling-are devised for computing a free-energy functional from a particle-based description. They can be employed to (i) derive the non-bonded free-energy functional of a soft coarse-grained model from a more detailed computational model or to (ii) derive a field-theoretic description from a particle-based model. Moreover, different strategies for accurately calculating free energies of self-assembled systems are described and selected applications presented.
\end{abstract}

Keywords Computer simulation · Self-consistent field theory $\cdot$ Block copolymers

\section{Introduction}

Amphiphiles are molecules that are composed of two or more distinct building blocks, which prefer different environments. Examples include biological lipid molecules, which contain

M. Müller ( $\varangle)$

Institut für Theoretische Physik, Georg-August-Universität, Friedrich-Hund-Platz 1, 37077 Göttingen,

Germany

e-mail: mmueller@theorie.physik.uni-goettingen.de 
a hydrophilic head and a hydrophobic tail, or block copolymers, which consist of two linear homopolymers that are covalently bound at their ends. Macroscopic phase separation between the different building blocks is prevented by the molecular connectivity. Instead, the molecules self-assemble into spatially modulated periodic phases with a characteristic length scale that is dictated by the size of the molecules [1]. This typical elementary length scale of a unit cell of the periodic morphology ranges from a few nanometers for biological lipids (e.g., the thickness of a bilayer membrane) to a few tens of nanometers for synthetic block copolymer materials. On the one hand, the time and length scales that are involved in structure formation - tens of nanometers and microseconds - cannot be systematically addressed by computational models with atomistic resolution. On the other hand, the small free-energy scale involved in these collective phenomena of soft matter poses a challenge for a coarse-grained description and requires the consideration of thermal fluctuations [2-11].

Recently, there has been much progress in studying the properties of amphiphilic systems with coarse-grained models. The advances are rooted in the development of predictive and computationally efficient soft coarse-grained models for polymer [12-21] and lipid systems $[5,6,8,11,22-24]$, as well as the development of simulation techniques [15, 25-31]. In this paper we review some computational aspects of soft coarse-grained models and discuss the relation of these particle-based models to a field-theoretic description. Our manuscript is arranged as follows: First, we will describe soft coarse-grained models that have been employed to investigate the universal aspects of structure formation in block copolymer systems. We will motivate their relevance, discuss how the model parameters can be related to experimental realizations, and explain their advantages for studying fluctuation effects. In particular, we will explore how to relate this class of particle-based models to a fieldtheoretic description. Some limitations due to the softness of the interactions are subsequently discussed. Second, we will highlight recent techniques to measure free energies of self-assembled systems [26, 28-30, 32-34]. Our contribution finishes with a brief summary and an outlook on open questions.

\section{Soft Coarse-Grained Models for Dense Amphiphilic Systems}

\subsection{Motivation: Universality of the Structure and Thermodynamics of Amphiphilic Systems}

One beautiful characteristics of amphiphilic systems consists in the universality of their qualitative characteristics [1,6]. System that markedly differ in their chemical structure and microscopic interactions between the amphiphilic blocks exhibit qualitatively similar behavior. For instance, if the volume fraction of the two building blocks is roughly equal, the material will self-assemble into a lamellar structure, where planar sheet-like domains of the components alternate. Upon decreasing the volume fraction of one block, one often observes that the minority component forms cylinders that arrange on a hexagonal lattice. A further decrease of the volume fraction of the minority block results in spherical aggregates (e.g., micelles). This sequence of morphologies is observed in biological lipids in aqueous solution [35], where the repulsion arises from the hydrophobic effect, as well as synthetic block copolymers in a molten state [36-38], where simple van der Waals interactions give rise to self-assembly. The observed universality suggests that the qualitative features of structure formation on large time and length scales can be captured by simple coarse-grained models that only incorporate those interactions that are relevant for bringing about the self-assembly $[4,6]$. 
Conceptually, one can "derive" such a coarse-grained representation by grouping a small number of atoms in a chemically realistic description into one effective interaction center (segment), and calculate the effective interactions between those coarse-grained segments (bottom-up approach) [39-44]. This procedure is similar to the construction of Hamiltonians in renormalization group theory of critical phenomena [45-48]. In fact, for polymer materials, this analogy to the theory of phase transitions can be made explicit due to the scale separation between the atomic repeat units and the properties of a polymer of high molecular weight in a melt or solution, and it has been exploited to investigate the structure of polymer solutions [49, 50].

The so-derived effective interactions depend on the thermodynamic state of the original system, i.e., they are free energies rather than energies [51-53]. Moreover, the more atoms are lumped together into a coarse-grained segment, the softer are the interactions among the coarse-grained entities. This reduction of the energy scale can be illustrated by the excluded volume interactions. On the microscopic scale, atoms cannot overlap and their interaction is characterized by a harsh excluded volume (Pauli repulsion). If one defines the center of mass of a collection of a large number of atoms as a coarse-grained degree of freedom, then these coarse-grained centers of mass may overlap, because the concomitant collections of atoms can interdigitate without violating the microscopic excluded-volume constraint. Thus their interaction is given by a much softer repulsion $[12,54,55]$ that merely limits density fluctuations [56]. Likewise, the energy scale of a covalent bond between two adjacent atoms along the backbone of a polymer molecule is on the order of electron Volt, while the typical energy of the interaction between two neighboring coarse-grained segments is only on the order of the thermal energy scale, $k_{B} T$.

\subsection{Soft Interactions-A Necessity}

Soft interactions on a mesoscopic length scale, which is much larger than the size of an atom but smaller than the entire extension of the amphiphilic molecule, naturally arise as a consequence of coarse-graining. In this subsection we argue that soft volume interactions, which allow for an overlap between coarse-grained segments, are necessary to capture an important property of polymer materials - the invariant degree of polymerization, $\overline{\mathcal{N}}$. This quantity characterizes the degree of interdigitation of molecules, and it is defined via

$$
\overline{\mathcal{N}} \equiv\left(\frac{\rho_{\mathrm{o}}}{N} R_{\text {eо }}^{3}\right)^{2}
$$

where $\rho_{\mathrm{o}}$ denotes the number density of segments, $N$ stands for the number of coarsegrained interaction centers that describe a molecule, and $R_{\mathrm{eo}}$ is the mean-squared end-toend distance that characterizes the size of a molecule in a non-interacting system. Since in a dense polymer melt the molecular conformations of a flexible, long macromolecule obey random-walk statistics, $R_{\mathrm{eo}} \sim \sqrt{N}$, the invariant degree of polymerization is proportional to the number of segments, $\overline{\mathcal{N}} \sim N$. Note, however, that the universal properties of the Gaussian chain conformations do not depend on the discretization $N$ of the chain contour but the fractal shape of a molecule is completely characterized by the single length scale, $R_{\mathrm{eo}}$. Also $\overline{\mathcal{N}}$ is invariant under changing the number of effective segments $N$ that are used to model the molecular conformations because $\rho_{\mathrm{o}} / N$ is the number density of molecules. The physical significance of $\overline{\mathcal{N}}$ consists in quantifying with how many neighbors a given macromolecule interacts. In the limit that $\overline{\mathcal{N}} \rightarrow \infty$, one molecule interacts with many neighbors and fluctuations and correlations on the length scale of $R_{\text {eo }}$ and beyond become less important [57]. 
In this limit, the system can be accurately described by the self-consistent field theory. For finite $\overline{\mathcal{N}}$, however, fluctuation effects are important.

Fluctuation and correlation effects are important for a variety of equilibrium properties [57]:

- Fluctuations in the vicinity of the critical point of demixing in polymer blends [58-63] or the order-disorder transition in block copolymer system [64, 65] shift the location of phase transitions and may change their qualitative character.

- Interfacial fluctuations (capillary waves) of the internal $A B$ interfaces in binary blends or block copolymers or the surfaces of polymer solutions and melts [66-68] broaden apparent interfacial profiles measured in experiments or extracted from simulations.

- The correlation hole in the intermolecular pair correlation function gives rise to important corrections to the Gaussian chain behavior in polymer melts in the bulk and thin films [69-77] and, additionally, leads to deviations of the single-chain dynamics from the Rouse model $[77,78]$.

The strength of these effects decreases with a power of $\overline{\mathcal{N}}$. Typical values of $\overline{\mathcal{N}}$ are on the order of $10^{4}$ for copolymer materials; values are smaller for biological lipid systems and they are often larger for mixtures of two homopolymers. Since computer simulations play an important role in assessing the quality of the mean-field approximation invoked in analytic or numerical calculations, e.g., the self-consistent field theory, and exploring the consequences of fluctuations, it is important for a coarse-grained computational model to describe a realistic value of $\overline{\mathcal{N}}$ that corresponds to experimental systems.

Modeling large values of $\overline{\mathcal{N}}=\left(\rho_{\mathrm{o}} b^{3}\right)^{2} N$ with computational representations that include excluded volume interactions between the coarse-grained segments, one faces a formidable challenge. In models like the Flory-Huggins representation of a polymer on a simple cubic lattice $[79,80]$, the bond fluctuation model $[59,81-83]$ or a Lennard-Jones bead-spring model $[67,84,85]$, the size of a segment, $\sigma$, as defined via the range of the harsh repulsive interactions, and the statistical segment length, $b \equiv R_{\mathrm{eo}} / \sqrt{N}$, are comparable, $\sigma \approx b$. Moreover, the segment density of a polymer fluid cannot be increased significantly beyond $\rho_{\mathrm{o}} \sigma^{3} \approx 1$, because the liquid of segments either crystallizes into a solid or it vitrifies into a glass. Thus, in order to model a polymer liquid, $\rho_{\mathrm{o}} b^{3} \lesssim 1$, and a value of $\overline{\mathcal{N}}=10^{4}$ requires a large number of segments per chain, $N \approx 10^{4}$.

The characteristic length scale of the self-assembled morphology is set by the molecular extension of a few $R_{\mathrm{eo}}$. A small system of linear dimension $L=R_{\mathrm{eo}}$ is comprised of $n=$ $\rho_{\mathrm{o}} L^{3}=N \sqrt{\overline{\mathcal{N}}}\left(L / R_{\mathrm{eo}}\right)^{3} \approx N^{3 / 2}=10^{6}$ effective segments. In a dense melt of these long molecules, the chains will reptate [86, 87], and the time to diffuse a distance $R_{\text {eo }}$ scales like $\tau=\tau_{\mathrm{o}} N^{3}$ where $\tau_{\mathrm{o}}$ is an $N$-independent microscopic time scale. To follow the system over one characteristic time one needs about $N^{9 / 2}=10^{18}$ elementary moves.

If the harsh excluded volume interaction is replaced by a soft repulsion, one will eliminate the constraint $\rho_{\mathrm{o}} b^{3} \lesssim 1$, because solidification or vitrification can be avoided. In this case, one can choose a large segment density, $\rho_{\mathrm{o}} b^{3} \sim \sqrt{\overline{\mathcal{N}}}$. For instance, choosing $\rho_{\mathrm{o}} b^{3}=18$, we can model a value of $\overline{\mathcal{N}}=10^{4}$ by using $N=31$ segments along the molecular contour. This discretization of the molecular architecture is still sufficient to capture the characteristics of the random-walk-like conformations on the scale $R_{\mathrm{eo}}$. Within the soft coarse-grained model, a system of size $L=R_{\text {eo }}$ contains only 3200 segments. Moreover, these non-entangled polymers obey Rouse dynamics with a relaxation time $\tau=\tau_{\mathrm{o}} N^{2}$. Thus the simulations require only $N^{3} \sqrt{\overline{\mathcal{N}}} \approx 3 \cdot 10^{8}$ elementary moves, which is 11 orders of magnitude less than in models, where excluded volume is enforced on the scale of a segment. 
For this reason, soft coarse-grained models are very efficient in describing polymer systems with a realistically large value of $\overline{\mathcal{N}}$ and allow us to study collective phenomena on the length scale of $R_{\mathrm{eo}}$ and beyond. This ability can be traced back to the rather coarse representation of the molecular contour and the concomitant large number of monomeric repeat units that are lumped into an effective coarse-grained segment.

\subsection{Bonded and Non-bonded Interactions}

We consider a soft coarse-grained model that describes $n$ polymers in a volume, $V$. Let $\left\{\mathbf{r}_{i, s}\right\}$ denotes the set of segment coordinates that completely specifies the configuration of our system. The index $i=1, \ldots, n$ runs over the number of molecules, and $s=1, \ldots, N$ labels the $N$ effective segments per chain molecule that describe the molecular conformation on the coarse-grained scale.

In the following, we distinguish between bonded interactions, which define the molecular shape and its fluctuations, and non-bonded interactions. The latter give rise to (i) the near incompressibility of a dense melt and (ii) the repulsion between the different segment species, $A$ and $B$, which drives the self-assembly.

The bonded interactions, $\mathcal{H}_{\mathrm{b}}\left(\left\{\mathbf{r}_{i, s}\right\}\right)$, of the coarse-grained model can be derived by enforcing that the coarse-grained description reproduces the distributions of distances along the molecular backbone [39]. In the limit that one coarse-grained segment is comprised of a large number of microscopic repeat units along a flexible polymer, the central limit theorem implies that the distance between neighboring coarse-grained segments is Gaussian distributed. Such a Gaussian distribution of coarse-grained bond lengths naturally gives rise to a bead-spring Hamiltonian introduced by Edwards [88]

$$
\frac{\mathcal{H}_{\mathrm{b}}\left(\left\{\mathbf{r}_{i, s}\right\}\right)}{k_{B} T}=\frac{3(N-1)}{2 R_{\mathrm{eo}}^{2}} \sum_{s=1}^{N-1}\left|\mathbf{r}_{i, s+1}-\mathbf{r}_{i, s}\right|^{2}
$$

In the non-interacting system, this bonded free energy leads to a Gaussian distribution of the coarse-grained bond lengths and the end-to-end distance

$$
\left\langle\left|\mathbf{r}_{i, s+1}-\mathbf{r}_{i, s}\right|^{2}\right\rangle=\frac{R_{\mathrm{eo}}^{2}}{N-1} \quad \text { and } \quad\left\langle\left|\mathbf{r}_{i, N}-\mathbf{r}_{i, 1}\right|^{2}\right\rangle=R_{\mathrm{eo}}^{2}
$$

This discretized Gaussian chain model captures the molecular conformations of long flexible polymers and their fluctuations on large length scales. Note that the large-scale conformations are complete described by the coarse-grained parameter $R_{\mathrm{eo}}$, i.e., the coarse-grained description of the chain conformations is invariant under changing the discretization $N$ of the molecular contour. Such a coarse-grained representation of the molecular architecture will be appropriate, if the characteristic length scale of the system, e.g., the width of the interface between microphase-separated domains, is larger than the smallest length scale, $b=R_{\mathrm{eo}} / \sqrt{N}$, on which the molecular contour is resolved. This condition results in a lower bound for the discretization $N$. In lipid systems or polymer systems with extremely large incompatibility between the species, however, the scale of spatial inhomogeneity may become comparable to the size of a monomeric repeat unit. In this case, the coarse-grained model has to incorporate further details of the local molecular architecture and liquid structure (cf. Sect. 2.8). As a first step, one can introduce a bending potential between bonds that allows to describe the cross-over from a rigid rod-like behavior on very short length scales to the Gaussian conformations of long macromolecules on large scales. Such a bending potential does not only parameterize the average local geometry of the molecule but it also reduces 
the conformational entropy by restricting fluctuations. Several systematic coarse-graining schemes, e.g., Boltzmann inversion [89] or force matching [90, 91], have been devised to derive augmented bonded free energies from a microscopic description, which includes details of the molecular architecture on short length scales (e.g., bond-length, bond-angle, or torsional potentials).

The non-bonded interactions, which capture the repulsion between the different segment species and the limited compressibility resulting from the excluded volume of the microscopic model, can be described by an excess free-energy functional $\mathcal{F}_{\mathrm{nb}}\left[\phi_{A}, \phi_{B}\right]$ of the local densities, $\phi_{A}(\mathbf{r})$ and $\phi_{B}(\mathbf{r})$, of the two segment species [11, 15, 25]. This general approach can benefit from abiding knowledge of classical density functional theory for liquids, and it allows for a great flexibility in describing a variety of phenomena, e.g., phase separation in dense multi-component melts [17, 29, 92, 93], liquid-vapor coexistence [15, 94, 95], and local packing effects of the fluid of segments or the main transition from a fluid to a gel phase in lipid bilayers [22].

To retain a particle-based description, we express the local densities through the positions of the coarse-grained segments. Schematically, one defines [96]

$$
\hat{\phi}_{A}\left(\mathbf{r} \mid\left\{\mathbf{r}_{i, s}\right\}\right)=\frac{1}{\rho_{\mathrm{o}}} \sum_{i=1}^{n} \sum_{s=1}^{N} \gamma_{i, s} \delta\left(\mathbf{r}-\mathbf{r}_{i, s}\right)
$$

where $\gamma_{i, s}=1$ if the $s^{\text {th }}$ segment of molecule $i$ is of type $A$ and 0 otherwise. A similar expression holds for the microscopic $B$ density. The "hat" indicates that the densities are functions of the segment coordinates. Using such a relation between the positions of the coarse-grained segments and the local densities, one can express the excess free energy as a function of the segment coordinates [25, 97]

$$
\mathcal{H}_{\mathrm{nb}}\left(\left\{\mathbf{r}_{i}\right\}\right) \equiv \mathcal{F}_{\mathrm{nb}}\left[\hat{\phi}_{A}\left(\mathbf{r} \mid\left\{\mathbf{r}_{i, s}\right\}\right), \hat{\phi}_{B}\left(\mathbf{r} \mid\left\{\mathbf{r}_{i, s}\right\}\right)\right]
$$

With these definitions the partition function of our soft coarse-grained model in the canonical ensemble is given by

$$
Z=\int \mathcal{D}\left[\left\{\mathbf{r}_{i, s}\right\}\right] \exp \left(-\frac{\mathcal{H}_{\mathrm{b}}+\mathcal{H}_{\mathrm{nb}}}{k_{B} T}\right)
$$

where $\mathcal{D}\left[\left\{\mathbf{r}_{i, s}\right\}\right] \equiv 1 /\left(n ! \Lambda_{T}^{3 n N}\right) \prod_{i=1}^{n} \prod_{s=1}^{N} \mathrm{~d}^{3} \mathbf{r}_{i, s}$ sums over all conformations and $\Lambda_{T}$ denotes the thermal de-Broglie wave length. The equilibrium properties of the so-defined soft coarse-grained model can be studied by a variety of computer simulation techniques including Monte Carlo simulation, Brownian dynamics or Dissipative Particle Dynamics.

In order to differentiate between the role of bonded and non-bonded contributions to the model interactions, one can rewrite the canonical partition function in the form

$$
\begin{aligned}
Z & =\int \mathcal{D}\left[\phi_{A}, \phi_{B}\right] \mathcal{D}\left[\left\{\mathbf{r}_{i, s}\right\}\right] e^{-\frac{\mathcal{H}_{\mathrm{b}}\left(\left\{\mathbf{r}_{i, s}\right\}\right)+\mathcal{F}_{\mathrm{n}}\left[\phi_{A}, \phi_{B}\right]}{k_{B} T}} \delta\left(\phi_{A}-\hat{\phi}_{A}\right) \delta\left(\phi_{B}-\hat{\phi}_{B}\right) \\
& =\int \mathcal{D}\left[\phi_{A}, \phi_{B}\right] e^{\frac{-\mathcal{F}_{\mathrm{nb}}\left[\phi_{A}, \phi_{B}\right]}{k_{B} T}} \int \mathcal{D}\left[\left\{\mathbf{r}_{i, s}\right\}\right] e^{-\frac{\mathcal{H}_{\mathrm{b}}\left(\left(\mathbf{r}_{i, s}\right\}\right)}{k_{B} T}} \delta\left(\phi_{A}-\hat{\phi}_{A}\right) \delta\left(\phi_{B}-\hat{\phi}_{B}\right) \\
& =\int \mathcal{D}\left[\phi_{A}, \phi_{B}\right] e^{\frac{-\mathcal{F}_{\mathrm{nb}}\left[\phi_{A}, \phi_{B}\right]}{k_{B} T}} e^{\frac{\mathcal{S}\left[\phi_{A}, \phi_{B}\right]}{k_{B}}} \text { with } \\
\mathcal{S}\left[\phi_{A}, \phi_{B}\right] & \equiv k_{B} \ln \int \mathcal{D}\left[\left\{\mathbf{r}_{i, s}\right\}\right] e^{-\frac{\mathcal{H}_{\mathrm{b}}\left(\left\{\mathbf{r}_{i, s}\right\}\right)}{k_{B} T}} \delta\left(\phi_{A}-\hat{\phi}_{A}\right) \delta\left(\phi_{B}-\hat{\phi}_{B}\right)
\end{aligned}
$$


Equations (7) and (8) explicitly establish a mapping from a particle-based description to a continuum field-theoretic one, where the collective densities, $\phi_{A}$ and $\phi_{B}$, rather than the segment coordinates, $\left\{\mathbf{r}_{i, s}\right\}$, are the fundamental degrees of freedom. The latter expression, (8), identifies $\mathcal{S}\left[\phi_{A}, \phi_{B}\right]$ as the entropy loss of the non-interacting molecules due to the density constraints, $\phi_{A}(\mathbf{r})=\hat{\phi}_{A}\left(\mathbf{r} \mid\left\{\mathbf{r}_{i, s}\right\}\right)$ and $\phi_{B}(\mathbf{r})=\hat{\phi}_{B}\left(\mathbf{r} \mid\left\{\mathbf{r}_{i, s}\right\}\right)$. It is comprised of two contribution: The entropy, $\mathcal{S}_{\mathrm{id}}$, of an ideal gas of non-bonded segments that accounts for the combinatorial entropy of distributing the segments in space and an excess entropy, $\mathcal{S}_{\mathrm{b}}$, that arises from the bonding of the segments into polymers and the conformational changes of the polymers with a spatially inhomogeneous density distribution.

$$
\begin{aligned}
\mathcal{S}\left[\phi_{A}, \phi_{B}\right]= & \mathcal{S}_{\mathrm{id}}\left[\phi_{A}, \phi_{B}\right]+\mathcal{S}_{\mathrm{b}}\left[\phi_{A}, \phi_{B}\right] \\
\mathcal{S}_{\mathrm{id}}\left[\phi_{A}, \phi_{B}\right]= & -k_{B} \rho_{\mathrm{o}} \int \mathrm{d}^{3} \mathbf{r}\left(\phi_{A}(\mathbf{r})\left[\ln \left(\phi_{A}(\mathbf{r}) \rho_{\mathrm{o}} \Lambda_{T}^{3}\right)-1\right]\right. \\
& \left.+\phi_{B}(\mathbf{r})\left[\ln \left(\phi_{B}(\mathbf{r}) \rho_{\mathrm{o}} \Lambda_{T}^{3}\right)-1\right]\right) \\
\mathcal{S}_{\mathrm{b}}\left[\phi_{A}, \phi_{B}\right]= & k_{B} \ln \int \overline{\mathcal{D}}\left[\left\{\mathbf{r}_{i, s}\right\}\right] e^{-\frac{\left.\mathcal{H}_{\mathrm{b}}\left(\mathbf{r}_{i, s}\right\}\right)}{k_{B} T}} \delta\left(\phi_{A}-\hat{\phi}_{A}\right) \delta\left(\phi_{B}-\hat{\phi}_{B}\right)
\end{aligned}
$$

where $\overline{\mathcal{D}}\left[\left\{\mathbf{r}_{i, s}\right\}\right]$ denotes the configurational integral for distinguishable particles.

In a one-component melt of very long flexible homopolymers, where the Ground-State Approximation (GSA) is appropriate, $\mathcal{S}$ corresponds to the Lifshitz entropy [98]

$$
-\frac{\mathcal{S}^{\text {homo }}[\phi]}{k_{B} \sqrt{\overline{\mathcal{N}}}} \approx \frac{R_{\mathrm{eo}}^{2}}{24} \int \frac{\mathrm{d}^{3} \mathbf{r}}{R_{\mathrm{eo}}^{3}} \frac{|\nabla \phi|^{2}}{\phi} \quad(\mathrm{GSA})
$$

If the variation of the densities has a small amplitude, $|\phi(\mathbf{r})-1| \ll 1$, one can use the Random-Phase Approximation (RPA) to calculate the entropy, $\mathcal{S}$, of a spatially inhomogeneous configuration [99]. For a one-component homopolymer system, one obtains the entropy functional

$$
-\frac{\mathcal{S}^{\text {homo }}[\phi]}{k_{B} \sqrt{\overline{\mathcal{N}}}} \approx \frac{V}{2 R_{\mathrm{eo}}^{3}} \sum_{\mathbf{q}} \frac{|\phi(\mathbf{q})|^{2}}{S(\mathbf{q}) / N} \quad(\mathrm{RPA})
$$

where

$$
\phi(\mathbf{q})=\frac{1}{V} \int \mathrm{d}^{3} \mathbf{r} e^{-i \mathbf{q r}} \phi(\mathbf{r})
$$

is the Fourier transform of the normalized density. The collective structure factor, $S(\mathbf{q})$, of the non-interacting system (cf. (8)) is identical to the single-chain structure factor, which is given by the Debye function, $g$

$$
\begin{aligned}
\frac{S(\mathbf{q})}{N} & =g(x)=\frac{2}{x^{2}}\left[e^{-x}-1+x\right] \quad \text { with } x=\frac{\left(q R_{\mathrm{eo}}\right)^{2}}{6} \\
& \approx 1-\frac{\left(q R_{\mathrm{eo}}\right)^{2}}{18}+\cdots \quad \text { for }\left(q R_{\mathrm{eo}}\right)^{2} \rightarrow 0
\end{aligned}
$$


Thus, in the limit of small amplitude variations and $\left(q R_{\mathrm{eo}}\right)^{2} \rightarrow 0$, the entropy of a homopolymer system takes the form (up to an additive constant)

$$
-\frac{\mathcal{S}^{\text {homo }}[\phi]}{k_{B} \sqrt{\overline{\mathcal{N}}}} \approx \int \frac{\mathrm{d}^{3} \mathbf{r}}{R_{\mathrm{eo}}^{3}}\left[\frac{1}{2}[\phi(\mathbf{r})-1]^{2}+\frac{R_{\mathrm{eo}}^{2}}{36}|\nabla \phi(\mathbf{r})|^{2}\right]
$$

In this limit, the combinatorial contribution to the entropy of the fluid of non-bonded segments can be expanded as follows

$$
\begin{aligned}
-\frac{\mathcal{S}_{\mathrm{id}}^{\text {homo }}[\phi]}{k_{B} \sqrt{\overline{\mathcal{N}}}} & =N \int \frac{\mathrm{d}^{3} \mathbf{r}}{R_{\mathrm{eo}}^{3}} \phi(\mathbf{r})\left[\ln \left(\phi(\mathbf{r}) \rho_{\mathrm{o}} \Lambda_{T}^{3}\right)-1\right] \\
& \approx N \int \frac{\mathrm{d}^{3} \mathbf{r}}{R_{\mathrm{eo}}^{3}} \frac{1}{2}[\phi(\mathbf{r})-1]^{2}+\text { constants } \quad(\mathrm{RPA})
\end{aligned}
$$

and we obtain the excess entropy (up to an additive constant)

$$
-\frac{\mathcal{S}_{\mathrm{b}}^{\text {homo }}[\phi]}{k_{B} \sqrt{\overline{\mathcal{N}}}}=\int \frac{\mathrm{d}^{3} \mathbf{r}}{R_{\mathrm{eo}}^{3}}\left[-\frac{N-1}{2}[\phi(\mathbf{r})-1]^{2}+\frac{R_{\mathrm{eo}}^{2}}{36}|\nabla \phi(\mathbf{r})|^{2}\right] \quad(\mathrm{RPA})
$$

The first term quantifies the change of the combinatorial entropy upon connecting $N$ segments to a chain molecule, while the second contribution stems from the alteration of the molecular conformations by the inhomogeneous environment.

In computer simulations, the entropy functional is not directly accessible but its functional derivative with respect to the local density - the chemical potential — can be measured, and computational strategies will be discussed in the next Sect. 2.4. Within the RPA, the chemical potential for a spatially inhomogeneous polymer melt takes the form:

$$
\begin{aligned}
\mu(\mathbf{r}) & \equiv-T \frac{\delta S}{\delta \phi(\mathbf{r})} \\
\frac{\mu(\mathbf{r}) R_{\mathrm{eo}}^{3}}{k_{B} T \sqrt{\overline{\mathcal{N}}}} & \approx \phi(\mathbf{r})-1-\frac{R_{\mathrm{eo}}^{2}}{18} \Delta \phi(\mathbf{r}) \quad(\mathrm{RPA})
\end{aligned}
$$

For a blend of two homopolymers, one can assume that composition fluctuations cost much less free energy than variations of the total density of the nearly incompressible system, i.e., $\phi_{A}(\mathbf{r})+\phi_{B}(\mathbf{r})=1$. The spatially varying densities can be described by the order parameter $m(\mathbf{r}) \equiv \phi_{A}(\mathbf{r})-\phi_{B}(\mathbf{r})$, and one obtains within RPA [99]:

$$
-\frac{\mathcal{S}\left[\phi_{A}, \phi_{B}\right]}{k_{B} \sqrt{\overline{\mathcal{N}}}}=\frac{V}{2 R_{\text {eo }}^{3}} \sum_{\mathbf{q}} \frac{|m(\mathbf{q})|^{2}}{4 S(\mathbf{q}) / N}
$$

The structure factor, $S(\mathbf{q})$, of composition fluctuations in an incompressible but otherwise ideal mixture is given by

$$
\frac{N}{S(\mathbf{q})}=\frac{1}{\bar{\phi}_{A} g_{A}(\mathbf{q})}+\frac{1}{\bar{\phi}_{B} g_{B}(\mathbf{q})}
$$

where $\bar{\phi}_{A}$ and $\bar{\phi}_{B}=1-\bar{\phi}_{A}$ denote the average volume fraction of $A$ and $B$ segments and $g_{A}$ and $g_{B}$ are the Debye functions of the two types of homopolymers, respectively. For a symmetric blend, where both homopolymer species have the same molecular extension, 
$R_{\mathrm{eo}}$, and $\bar{\phi}_{A}=\bar{\phi}_{B}=1 / 2$, the RPA predicts in the limit of small amplitude variations and $\left(q R_{\mathrm{eo}}\right)^{2} \rightarrow 0$

$$
-\frac{\mathcal{S}^{\text {sym.blend }}[m]}{k_{B} \sqrt{\overline{\mathcal{N}}}} \approx \int \frac{\mathrm{d}^{3} \mathbf{r}}{R_{\mathrm{eo}}^{3}}\left[\frac{1}{2}\left(\frac{m}{2}\right)^{2}+\frac{R_{\mathrm{eo}}^{2}}{36}\left(\frac{\nabla m}{2}\right)^{2}\right]
$$

In case of a diblock copolymer, (23) still holds and the structure factor is given by [100]

$$
\frac{S(q)}{N}=\frac{g(1, x)}{g(f, x) g(1-f, x)-\frac{1}{4}[g(1, x)-g(f, x)-g(1-f, x)]^{2}}
$$

with $g(f, x)=\frac{2}{x^{2}}\left[e^{-f x}-1+f x\right]$ and $x=\frac{\left(q R_{\mathrm{eo}}\right)^{2}}{6}$

where $f$ is the composition of the block copolymer. For a symmetric diblock copolymer, $f=1 / 2$, this expression simplifies to

$$
\begin{aligned}
\frac{N}{S(\mathbf{q})} & =\frac{1}{g(1 / 2, x)-\frac{1}{4} g(1, x)} \\
& \approx \frac{24}{x}+7.1+2 x \quad \text { with } x=\frac{\left(q R_{\mathrm{eo}}\right)^{2}}{6}
\end{aligned}
$$

The latter approximation captures the leading-order behavior in the limit $x \rightarrow 0$ and $x \rightarrow \infty$ [101] and leads to the expression by Ohta and Kawasaki

$$
\begin{aligned}
-\frac{\mathcal{S}^{\text {sym.cop }}\left[m=\phi_{A}-\phi_{B}\right]}{k_{B} \sqrt{\overline{\mathcal{N}}}} \approx & \int \frac{\mathrm{d}^{3} \mathbf{r}}{R_{\mathrm{eo}}^{3}}\left[7.1\left(\frac{m}{2}\right)^{2}+\frac{R_{\mathrm{eo}}^{2}}{24}\left(\frac{\nabla m}{2}\right)^{2}\right] \\
& +18 \int \frac{\mathrm{d}^{3} \mathbf{r}}{R_{\mathrm{eo}}^{3}} \frac{\mathrm{d}^{3} \mathbf{r}^{\prime}}{R_{\mathrm{eo}}^{3}} \frac{m(\mathbf{r})}{2} R_{\mathrm{eo}} G\left(\mathbf{r}-\mathbf{r}^{\prime}\right) \frac{m\left(\mathbf{r}^{\prime}\right)}{2}
\end{aligned}
$$

where the integral kernel obeys the relation $\Delta G(\mathbf{r})=-\delta(\mathbf{r})$. For an incompressible system, one obtains for the chemical potential difference (exchange potential), which is thermodynamically conjugated to the order parameter, $m$

$$
\mu_{A}(\mathbf{r})-\mu_{B}(\mathbf{r}) \equiv-T \frac{\delta \mathcal{S}}{\delta \phi_{A}(\mathbf{r})}+T \frac{\delta \mathcal{S}}{\delta \phi_{B}(\mathbf{r})}=-2 T \frac{\delta \mathcal{S}}{\delta m(\mathbf{r})}
$$

where the variation $\delta \phi_{A}(\mathbf{r})$ does not fulfill the incompressibility constraint, i.e., $\delta \phi_{A}(\mathbf{r})$ and $\delta \phi_{B}(\mathbf{r})$ are independent. The RPA, (23), results in

$$
\begin{aligned}
\mu_{A}(\mathbf{q})-\mu_{B}(\mathbf{q}) & =-2 \frac{T}{V} \frac{\partial \mathcal{S}}{\partial m(-\mathbf{q})} \\
\frac{\left[\mu_{A}(\mathbf{q})-\mu_{B}(\mathbf{q})\right] R_{\mathrm{eo}}^{3}}{k_{B} T \sqrt{\overline{\mathcal{N}}}} & \approx \frac{1}{2} \frac{m(\mathbf{q})}{S(\mathbf{q}) / N} \quad(\mathrm{RPA}
\end{aligned}
$$

For a variation of the composition with a single wave vector, $q_{x}$, e.g., $m(\mathbf{r})=A \cos \left(q_{x} x\right)$ or $m(\mathbf{q})=\frac{A}{2}\left(\delta_{\mathbf{q}, q_{x}}+\delta_{\mathbf{q},-q_{x}}\right)$, the chemical potential takes the particularly simple form

$$
\frac{\left[\mu_{A}(\mathbf{r})-\mu_{B}(\mathbf{r})\right] R_{\mathrm{eo}}^{3}}{k_{B} T \sqrt{\overline{\mathcal{N}}}} \approx \frac{1}{2} \frac{m(\mathbf{r})}{S\left(q_{x}\right) / N}=\frac{N}{2 S\left(q_{x}\right)}\left[\phi_{A}(\mathbf{r})-\phi_{B}(\mathbf{r})\right]
$$

and contributions with different wave vectors superpose linearly within the RPA. 
More complex molecular architectures, e.g., branched polymers, multiblock, random or comb copolymers [102-110], give rise to more complex entropy functionals, $\mathcal{S}$, and it is of interest to determine those functionals without approximation by computer simulation of particle-based models.

\subsection{Bottom-Up Construction of a Soft Coarse-Grained Model: Constraint Partition Function}

In principle, one can derive a coarse-grained model from an underlying microscopic model. For the coarse-grained model to reproduce the same macroscopic equilibrium properties as the microscopic model, one must enforce that the partition functions of the two representations are identical. In the following, we require the stronger condition that the free energy of a given coarse-grained density distribution, $\phi_{A}(\mathbf{r})$ and $\phi_{B}(\mathbf{r})$, which is defined via the constraint partition function (see (37) below), is the same in the two representation (consistency).

In such a bottom-up approach, one starts out with a more microscopic model, which could be an atomistic representation or a less coarse-grained model of the system under investigation. Let $\left\{\mathbf{x}_{\alpha}\right\}$ denote these microscopic degrees of freedom and $E\left[\left\{\mathbf{x}_{\alpha}\right\}\right]$ the energy associated with a microscopic configuration. The index $\alpha$ runs over all microscopic degrees of freedom. Then, one defines the projection $\tilde{\mathbf{r}}_{i, s}\left(\left\{\mathbf{x}_{\alpha}\right\}\right)$ from the microscopic degrees of freedom $\left\{\mathbf{x}_{\alpha}\right\}$ onto the coarse-grained ones $\left\{\mathbf{r}_{i, s}\right\}$.

Formally, one can derive the coarse-grained model from the underlying microscopic model by performing a partial trace over all microscopic degrees of freedom compatible with a fixed density distribution.

$$
\begin{aligned}
Z= & \int \mathcal{D}\left[\phi_{A}, \phi_{B}\right] \exp \left(-\frac{\mathcal{F}_{\mathrm{nb}}\left[\phi_{A}, \phi_{B}\right]}{k_{B} T}\right) \exp \left(\frac{\mathcal{S}\left[\phi_{A}, \phi_{B}\right]}{k_{B}}\right) \\
= & \int \mathcal{D}\left[\phi_{A}, \phi_{B}\right] \mathcal{D}\left[\left\{\mathbf{x}_{\alpha}\right\}\right] e^{-\frac{E\left(\left\{\mathbf{x}_{\alpha}\right\}\right)}{k_{B} T}} \\
& \times \delta\left(\phi_{A}(\mathbf{r})-\hat{\phi}_{A}\left(\mathbf{r} \mid\left\{\tilde{\mathbf{r}}_{i, s}\left(\left\{\mathbf{x}_{\alpha}\right\}\right)\right\}\right)\right) \delta\left(\phi_{B}(\mathbf{r})-\hat{\phi}_{B}\left(\mathbf{r} \mid\left\{\tilde{\mathbf{r}}_{i, s}\left(\left\{\mathbf{x}_{\alpha}\right\}\right)\right\}\right)\right)
\end{aligned}
$$

We fulfill the requirement that the microscopic and the coarse-grained system have the same constraint partition function by defining the non-bonded interactions according to

$$
\begin{aligned}
\mathcal{F}_{\mathrm{nb}}\left[\phi_{A}, \phi_{B}\right]= & T \mathcal{S}\left[\phi_{A}, \phi_{B}\right] \\
& -k_{B} T \ln \int \mathcal{D}\left[\left\{\mathbf{x}_{\alpha}\right\}\right] e^{-\frac{E\left(\left\{\mathbf{x}_{\alpha}\right\}\right)}{k_{B} T}} \delta\left(\phi_{A}(\mathbf{r})-\hat{\phi}_{A}\left(\mathbf{r} \mid\left\{\tilde{\mathbf{r}}_{i, s}\left(\left\{\mathbf{x}_{\alpha}\right\}\right)\right\}\right)\right) \\
& \times \delta\left(\phi_{B}(\mathbf{r})-\hat{\phi}_{B}\left(\mathbf{r} \mid\left\{\tilde{\mathbf{r}}_{i, s}\left(\left\{\mathbf{x}_{\alpha}\right\}\right)\right\}\right)\right)
\end{aligned}
$$

i.e., the constraint partition function of the more microscopic model with a fixed coarsegrained density distribution, $\phi_{A}(\mathbf{r})$ and $\phi_{B}(\mathbf{r})$, has to be evaluated to obtain the second term of (37). This calculation yields the free-energy functional, $\mathcal{F}=\mathcal{F}_{\mathrm{nb}}-T \mathcal{S}$, of the fieldtheoretic description of the model, and the consistency requirement ensures that this freeenergy functional of the coarse-grained and of the more microscopic model are the same. Then, one has to compute the constraint partition function of the coarse-grained model in the absence of non-bonded interactions, (8), to obtain $\mathcal{S}$, and the difference yields the excess free-energy functional, $\mathcal{F}_{\mathrm{nb}}$, of the non-bonded interaction of the coarse-grained model. 
Thus the evaluation of constraint partition functions, e.g. (8) or (37), serves either to systematically derive $\mathcal{F}_{\mathrm{nb}}\left[\phi_{A}, \phi_{B}\right]$ for a coarse-grained model or to bridge between a particlebased description and a field-theoretic one, where the collective fields, $\phi_{A}(\mathbf{r})$ and $\phi_{B}(\mathbf{r})$ are the fundamental degrees of freedom.

There are several strategies for calculating the constraint partition functions, defined in (8) and (37), from particle-based simulations. The first method is based on force matching techniques that have originally been developed in the context of systematic coarse-graining of particle-based models [90, 91]. Recently, this approach has been formulated by Villet and Fredrickson for field-theoretic simulations [111], connecting two field-theoretic representation of a fluid with different spatial resolutions. Here we formulate this technique to bridge from a particle-based description to a continuum one, where one describes the system configuration by a free-energy functional that depends on the coarse-grained density fields, $\phi_{A}(\mathbf{r})$ and $\phi_{B}(\mathbf{r})$. The second method is a generalization of umbrella sampling from a single degree of freedom to a continuum field [21]. Both methods have in common that the free energy is not calculated directly but rather its derivative with respect to the continuum fields, $\phi_{A}$ and $\phi_{B}$.

\subsubsection{Field-Theoretic Force Matching Technique}

To simplify notation, we restrict ourselves to the calculation of the excess entropy, (11) but the techniques can be applied to (37) by replacing $-T \mathcal{S}$ by $\mathcal{F}=\mathcal{F}_{\mathrm{nb}}-T \mathcal{S}$ and $\mathcal{H}_{\mathrm{b}}$ by $\mathcal{H}=\mathcal{H}_{\mathrm{nb}}+\mathcal{H}_{\mathrm{b}}$

The functional derivative of (11) with respect to the local density $\phi_{A}$ is given by:

$$
\frac{\delta \mathcal{S}_{\mathrm{b}}\left[\phi_{A}, \phi_{B}\right]}{\delta \phi_{A}(\mathbf{r})}=k_{B} \frac{\int \overline{\mathcal{D}}\left[\left\{\mathbf{r}_{i, s}\right\}\right] e^{-\frac{\left.\mathcal{H}_{\mathrm{b}}\left(\mathbf{r}_{i, s}\right\}\right)}{k_{B} T}}\left[\frac{\delta}{\delta \phi_{A}(\mathbf{r})} \delta\left(\phi_{A}-\hat{\phi}_{A}\right)\right] \delta\left(\phi_{B}-\hat{\phi}_{B}\right)}{\int \overline{\mathcal{D}}\left[\left\{\mathbf{r}_{i, s}\right\}\right] e^{-\frac{\mathcal{H}_{\mathrm{b}}\left(\left(\mathbf{r}_{i, s}\right\}\right)}{k_{B} T}} \delta\left(\phi_{A}-\hat{\phi}_{A}\right) \delta\left(\phi_{B}-\hat{\phi}_{B}\right)}
$$

Let $\mathbf{r}_{i, s, \alpha}$ be the $\alpha$-coordinate of the $s^{\text {th }}$ segment on polymer $i$. The segment is chosen such that a movement of that segment in the Cartesian direction, $\alpha \in\{x, y, z\}$, alters the microscopic $A$ density at position $\mathbf{r}$, i.e.

$$
\left|\frac{\partial \hat{\phi}_{A}\left(\mathbf{r} \mid\left\{\mathbf{r}_{i, s}\right\}\right)}{\partial \mathbf{r}_{i, s, \alpha}}\right|>0
$$

In the following, we omit the dependence of $\hat{\phi}_{A}\left(\mathbf{r} \mid\left\{\mathbf{r}_{i, s}\right\}\right)$ on the set of segment coordinates, $\left\{\mathbf{r}_{i, s}\right\}$ and note that the derivative, $\partial \hat{\phi}_{A}\left(\mathbf{r} \mid\left\{\mathbf{r}_{i, s}\right\}\right) / \partial \mathbf{r}_{i, s, \alpha}$, does not depend on the coordinates of the other segments. From the definition of the microscopic densities, (4), we obtain

$$
\Delta L^{3} \frac{\delta}{\delta \phi_{A}(\mathbf{r})} \delta\left(\phi_{A}-\hat{\phi}_{A}\right)=-\delta\left(\phi_{A}-\hat{\phi}_{A}\right) \frac{1}{\frac{\partial \hat{\phi}_{A}(\mathbf{r})}{\partial \mathbf{r}_{i, s, \alpha}}} \frac{\partial}{\partial \mathbf{r}_{i, s, \alpha}} \ln \delta\left(\phi_{A}(\mathbf{r})-\hat{\phi}_{A}(\mathbf{r})\right)
$$

where $\Delta L^{3}$ is a microscopic volume element, and

$$
-T \frac{\delta \mathcal{S}_{\mathrm{b}}\left[\phi_{A}, \phi_{B}\right]}{\delta \phi_{A}(\mathbf{r})}=\frac{k_{B} T}{\Delta L^{3}}\left\langle\frac{1}{\frac{\partial \hat{\phi}_{A}(\mathbf{r})}{\partial \mathbf{r}_{i, s, \alpha}}} \frac{\partial}{\partial \mathbf{r}_{i, s, \alpha}} \ln \delta\left(\phi_{A}(\mathbf{r})-\hat{\phi}_{A}(\mathbf{r})\right)\right\rangle_{\phi_{A}, \phi_{B}}
$$

where the brackets denote the constraint average

$$
\langle\cdots\rangle_{\phi_{A}, \phi_{B}} \equiv e^{-\frac{\mathcal{S}_{b}\left[\phi_{A}, \phi_{B}\right]}{k_{B} T}} \int \overline{\mathcal{D}}\left[\left\{\mathbf{r}_{i, s}\right\}\right] e^{-\frac{\mathcal{H}_{\mathrm{b}}\left(\left(\mathbf{r}_{i, s}\right\}\right)}{k_{B} T}} \cdots \delta\left(\phi_{A}-\hat{\phi}_{A}\right) \delta\left(\phi_{B}-\hat{\phi}_{B}\right)
$$


Using integration by parts, one can show that

$$
\int \frac{\mathrm{d}^{3} \mathbf{r}}{\Delta L^{3}}\left\langle\frac{\partial}{\partial \mathbf{r}_{i, s, \alpha}} \ln \delta\left(\phi_{A}(\mathbf{r})-\hat{\phi}_{A}(\mathbf{r})\right)\right\rangle_{\phi_{A}, \phi_{B}}=\frac{1}{k_{B} T}\left\langle\frac{\partial \mathcal{H}_{\mathrm{b}}\left(\left\{\mathbf{r}_{i, s}\right\}\right)}{\partial \mathbf{r}_{i, s, \alpha}}\right\rangle_{\phi_{A}, \phi_{B}}
$$

In conjunction with (41), this relation yields

$$
\left\langle\frac{\partial \mathcal{H}_{\mathrm{b}}\left(\left\{\mathbf{r}_{i, s}\right\}\right)}{\partial \mathbf{r}_{i, s, \alpha}}\right\rangle_{\phi_{A}, \phi_{B}}=\int \mathrm{d}^{3} \mathbf{r} \frac{\partial \hat{\phi}_{A}(\mathbf{r})}{\partial \mathbf{r}_{i, s, \alpha}} \frac{\delta}{\delta \phi_{A}(\mathbf{r})}\left(-T \mathcal{S}_{\mathrm{b}}\left[\phi_{A}, \phi_{B}\right]\right)
$$

The above equation relates the average force,

$$
\left\langle\mathbf{K}_{A, \alpha}\left(\mathbf{r}_{i, s}\right)\right\rangle_{\phi_{A}, \phi_{B}} \equiv-\left\langle\partial \mathcal{H}_{\mathrm{b}}\left(\left\{\mathbf{r}_{i, s}\right\}\right) / \partial \mathbf{r}_{i, s, \alpha}\right\rangle_{\phi_{A}, \phi_{B}},
$$

which acts on a segment of type $A$ at position $\mathbf{r}_{i, s}$ in the constraint system, to the local excess of the chemical potential, $\mu_{\mathrm{b} A}(\mathbf{r}) \equiv-T \delta \mathcal{S}_{\mathrm{b}} / \delta \phi_{A}(\mathbf{r})$. Using the functional inverse define by

$$
\int \mathrm{d}^{3} \mathbf{r}_{i, s} \frac{\partial \hat{\phi}_{A}(\mathbf{r})}{\partial \mathbf{r}_{i, s, \alpha}}\left[\frac{\partial \hat{\phi}_{A}\left(\mathbf{r}^{\prime}\right)}{\partial \mathbf{r}_{i, s, \alpha}}\right]^{-1}=\delta\left(\mathbf{r}-\mathbf{r}^{\prime}\right)
$$

we can invert this relation and obtain

$$
\begin{aligned}
-T \frac{\delta \mathcal{S}_{\mathrm{b}}\left[\phi_{A}, \phi_{B}\right]}{\delta \phi_{A}\left(\mathbf{r}^{\prime}\right)} & =\int \mathrm{d}^{3} \mathbf{r}_{i, s}\left[\frac{\partial \hat{\phi}_{A}\left(\mathbf{r}^{\prime}\right)}{\partial \mathbf{r}_{i, s, \alpha}}\right]^{-1}\left\langle\frac{\partial \mathcal{H}_{\mathrm{b}}\left(\left\{\mathbf{r}_{i, s}\right\}\right)}{\partial \mathbf{r}_{i, s, \alpha}}\right\rangle_{\phi_{A}, \phi_{B}} \\
\mu_{\mathrm{b} A}\left(\mathbf{r}^{\prime}\right) & =-\int \mathrm{d}^{3} \mathbf{r}_{i, s}\left[\frac{\partial \hat{\phi}_{A}\left(\mathbf{r}^{\prime}\right)}{\partial \mathbf{r}_{i, s, \alpha}}\right]^{-1}\left\langle\mathbf{K}_{A, \alpha}\left(\mathbf{r}_{i, s}\right)\right\rangle_{\phi_{A}, \phi_{B}}
\end{aligned}
$$

Since the segment $(i, s)$ is chosen to contribute to the local $A$ density, $\gamma_{i, s}=1$ and the functional inverse takes the form

$$
\left[\frac{\partial \hat{\phi}_{A}\left(\mathbf{r}^{\prime}\right)}{\partial \mathbf{r}_{i, s, \alpha}}\right]^{-1}=-\rho_{\mathrm{o}} \Theta\left(\mathbf{r}_{\alpha}^{\prime}-\mathbf{r}_{i, s, \alpha}\right) \prod_{\beta \neq \alpha} \delta\left(\mathbf{r}_{\beta}^{\prime}-\mathbf{r}_{i, s, \beta}\right)
$$

where $\Theta$ is the Heaviside step function. This relation yields

$$
\mu_{\mathrm{b} A}\left(\mathbf{r}^{\prime}\right)=\rho_{\mathrm{o}} \int_{\mathbf{r}_{x}^{\prime}}^{\infty} \mathrm{d} x\left\langle K_{A, x}\left(x, \mathbf{r}_{y}^{\prime}, \mathbf{r}_{z}^{\prime}\right)\right\rangle_{\phi_{A}, \phi_{B}}
$$

and, after differentiation, we obtain the final result

$$
\nabla \mu_{\mathrm{b} A}(\mathbf{r})=\nabla\left[\mu_{A}(\mathbf{r})-\rho_{\mathrm{o}} k_{B} T \ln \phi_{A}(\mathbf{r})\right]=-\rho_{\mathrm{o}}\left\langle\mathbf{K}_{A}(\mathbf{r})\right\rangle_{\phi_{A}, \phi_{B}}
$$

This relation is the analog of the lowest-order Born-Green-Yvon relation [44, 112, 113] for the constraint system. Equation (50) has the simple interpretation that, in the constraint system, the excess chemical force density, $\nabla \mu_{\mathrm{b} A}(\mathbf{r})$, due to the constraint, and the density of internal forces $\rho_{\mathrm{o}} K_{A}(\mathbf{r})$ balance. Hence this strategy, which equates the force of the fieldtheoretic model to the force of the corresponding particle-based model, is termed forcematching. 
Using (10) we obtain for the gradient of the total chemical potential

$$
R_{\mathrm{eo}} \nabla \frac{\mu_{A}(\mathbf{r}) R_{\mathrm{eo}}^{3}}{k_{B} T \sqrt{\overline{\mathcal{N}}}}=N R_{\mathrm{eo}} \nabla \ln \phi_{A}(\mathbf{r})-N \frac{R_{\mathrm{eo}}}{k_{B} T}\left\langle\mathbf{K}_{A}(\mathbf{r})\right\rangle_{\phi_{A}, \phi_{B}}
$$

An alternative derivation of this relation within the field-theoretic formalism is given in the Appendix.

In the typical case, the left hand side of (51) is of order unity, while both terms on the right hand side are $\mathcal{O}(N)$. Thus the gradient of the chemical potential is obtained from the difference of two large numbers, and the situation is similar to the cancellation between bonded and non-bonded contributions that occurs when one calculates the pressure via the virial of all forces.

\subsubsection{Field-Theoretic Umbrella Sampling}

In general, however, it is difficult to strictly constrain the microscopic densities, $\hat{\phi}_{A}$ and $\hat{\phi}_{B}$, because it gives rise to sampling problems of the underlying particle-based model. In most computational schemes, ${ }^{1}$ the update of the system configurations does involve a change of the microscopic density but such a change will violate the constraint and, consequently, those configuration updates will be rejected.

In these cases, it is computationally convenient to restrain the fluctuations of the microscopic densities around $\phi_{A}$ and $\phi_{B}$ rather than to strictly constrain them [21]. To this end, one can soften the $\delta$-function constraint in (8) via [21, 115]

$$
\delta\left(\phi_{A}-\hat{\phi}_{A}\right) \sim \lim _{\lambda N \rightarrow \infty} \exp \left(-\frac{\sqrt{\overline{\mathcal{N}}} \lambda N}{2} \int \frac{\mathrm{d}^{3} \mathbf{r}}{R_{\mathrm{eo}}^{3}}\left[\phi_{A}(\mathbf{r})-\hat{\phi}_{A}\left(\mathbf{r} \mid\left\{\mathbf{r}_{i, s}\right\}\right)\right]^{2}\right)
$$

which is equivalent to an additional contribution to the non-bonded free-energy functional of the form

$$
\frac{\mathcal{F}_{\lambda N}}{k_{B} T \sqrt{\overline{\mathcal{N}}}}=\frac{\lambda N}{2} \int \frac{\mathrm{d}^{3} \mathbf{r}}{R_{\text {eo }}^{3}}\left(\left[\phi_{A}-\hat{\phi}_{A}\right]^{2}+\left[\phi_{B}-\hat{\phi}_{B}\right]^{2}\right)
$$

Using this representation of the $\delta$-function and defining the restraint average according to

$$
\langle\cdots\rangle_{\lambda N} \propto \lim _{\lambda N \rightarrow \infty} \int \mathcal{D}\left[\left\{\mathbf{r}_{i, s}\right\}\right] e^{-\frac{\mathcal{H}_{\mathrm{b}}\left(\left\{\mathbf{r}_{i, s}\right\}\right)}{k_{B} T}} \cdots e^{-\frac{\sqrt{\mathcal{N}} \lambda N}{2} \int \frac{\mathrm{d}^{3} \mathbf{r}}{R_{\mathrm{eO}}^{3}}\left(\left[\phi_{A}-\hat{\phi}_{A}\right]^{2}+\left[\phi_{B}-\hat{\phi}_{B}\right]^{2}\right)}
$$

where the constant of proportionality is chosen such that $\langle 1\rangle_{\lambda N}=1$, we can calculate the thermodynamic force [21]

$$
\frac{\mu_{A}(\mathbf{r}) R_{\mathrm{eo}}^{3}}{k_{B} T \sqrt{\overline{\mathcal{N}}}}=-\frac{R_{\mathrm{eo}}^{3}}{k_{B} \sqrt{\overline{\mathcal{N}}}} \frac{\delta \mathcal{S}_{\mathrm{b}}\left[\phi_{A}, \phi_{B}\right]}{\delta \phi_{A}(\mathbf{r})}=\lambda N\left\langle\phi_{A}(\mathbf{r})-\hat{\phi}_{A}\left(\mathbf{r} \mid\left\{\mathbf{r}_{i, s}\right\}\right)\right\rangle_{\lambda N}
$$

This strategy resembles umbrella sampling, where one restrains the fluctuations of "a" variable $\hat{\phi}_{A}$ by a harmonic umbrella potential and estimates the thermodynamic constraint force by the "displacement" $\phi_{A}-\left\langle\hat{\phi}_{A}\right\rangle_{\lambda N}$ of the restraint system from the reference value $\phi_{A}$. In

\footnotetext{
${ }^{1}$ Connectivity altering moves, which have been devised by Theodorou and co-workers [114], are a notable exception.
} 
order to obtain an accurate estimate of $\mu$, the coupling parameter $\lambda N$ must be chosen large. In this limit, the technique relies on measuring the small difference between the imposed density $\phi_{A}(\mathbf{r})$ and the thermal average, $\left\langle\hat{\phi}_{A}(\mathbf{r})\right\rangle$, that the restraint system adopts.

The field-theoretic umbrella sampling, (55), or the force-matching relation, (51), provide a computational strategy for estimating the chemical potential (or its gradient) from the restrained (or constraint) average. These techniques yield an unbiased estimate of the underlying free-energy functional that can be employed in conjunction with equation-free multiscale computations [116] or heterogeneous multiscale techniques [117-119] provided that the free-energy functional can be efficiently estimated.

To this end, one can make an Ansatz, $\mathcal{F}_{\text {trial }}\left[\phi_{A}, \phi_{B} \mid\left\{p_{i}\right\}\right]$, for the free-energy functional of the continuum description [21]. The functional form of this Ansatz shall be guided by symmetry considerations, and it will contain a small set of parameters, $\left\{p_{i}\right\}$, that characterize the microscopic model system. From such an Ansatz, one can calculate the chemical potential, $\mu_{A \text {,trial }}\left[\phi_{A}, \phi_{B} \mid\left\{p_{i}\right\}\right]$, or its gradient and, by comparing this expression to (55) or (51), one can estimate the parameters, $\left\{p_{i}\right\}$, of the free-energy functional, i.e., like in force-matching $[90,91]$ one minimizes

$$
\begin{aligned}
\chi_{\phi_{A}, \phi_{B}}^{2} & =\frac{R_{\mathrm{eo}}^{3}}{k_{B} T \sqrt{\overline{\mathcal{N}}}} \int \mathrm{d}^{3} \mathbf{r}\left[\mu_{A, \text { trial }}\left[\phi_{A}(\mathbf{r}), \phi_{B}(\mathbf{r}) \mid\left\{p_{i}\right\}\right]-\mu_{A}(\mathbf{r})\right]^{2} \\
& =\int \mathrm{d}^{3} \mathbf{r}\left[\frac{\mu_{A, \text { trial }}\left[\phi_{A}(\mathbf{r}), \phi_{B}(\mathbf{r}) \mid\left\{p_{i}\right\}\right] R_{\mathrm{eo}}^{3}}{k_{B} T \sqrt{\overline{\mathcal{N}}}}-\lambda N\left(\phi_{A}(\mathbf{r})-\left\langle\hat{\phi}_{A}(\mathbf{r})\right\rangle_{\lambda N}\right)\right]^{2}
\end{aligned}
$$

with respect to the set of parameters, $\left\{p_{i}\right\}$. This strategy allows for gauging the quality of the Ansatz by monitoring how $\chi_{\phi_{A}, \phi_{B}}^{2}$ and the parameters $\left\{p_{i}\right\}$ depend on the field configuration, $\phi_{A}$ and $\phi_{B}$, and for systematically improving the Ansatz.

\subsubsection{Numerical Examples}

First, we consider a homopolymer system with chain discretizations $N=32, \kappa_{0} N=0$, and $\chi_{\mathrm{o}} N=0$. Using $\lambda N=100$ we restrain the system to a density distribution $\phi(\mathbf{r})=$ $1+\varepsilon \cos \left(q_{x} x\right)$ with $\varepsilon=0.2$ and $q_{x} R_{\text {eo }}=\pi$ and $2 \pi$, respectively. In Fig. 1(a) we present the one-dimensional density profile, $\langle\hat{\phi}(x)\rangle$, observed in the restraint simulations and the imposed density profile, $\phi(\mathbf{r})$. Since $\lambda N$ is large, the two profiles are almost identical; the spatial variation of $\langle\hat{\phi}(x)\rangle$ has a slightly smaller amplitude than that of $\phi(\mathbf{r})$. The reduction of the amplitude is $1.4 \%$ and $4 \%$ for $q_{x} R_{\mathrm{eo}}=\pi$ and $2 \pi$, respectively.

From the difference of both profiles, one can estimate the chemical potential of the spatially inhomogeneous system according to (55), which is presented in Fig. 1(b). The simulation results are compared with the prediction of the RPA

$$
\frac{\mu(\mathbf{r}) R_{\mathrm{eo}}^{3}}{k_{B} T \sqrt{\overline{\mathcal{N}}}}=\frac{N}{2 S\left(q_{x}\right)}[\phi(\mathbf{r})-1] \quad(\mathrm{RPA})
$$

for a spatial variation with a single Fourier mode, where $S$ denotes the single-chain structure factor. This expression is valid for density variations of small amplitude. Alternatively, the simulation data can be compared to the expression

$$
\frac{\mu(\mathbf{r}) R_{\mathrm{eo}}^{3}}{k_{B} T \sqrt{\overline{\mathcal{N}}}}=\ln \phi(\mathbf{r})-\frac{R_{\mathrm{eo}}^{2}}{18} \Delta \phi(\mathbf{r})
$$





Fig. 1 Restraint simulation of a homopolymer system with $N=32, \kappa_{\mathrm{o}} N=0, \chi_{\mathrm{o}} N=0, \overline{\mathcal{N}}=128^{2}$, $\lambda N=100$, and first-order assignment (cf. (69)) using $\Delta L=R_{\mathrm{eo}} / 6$. (a) One-dimensional density profile, $\langle\hat{\phi}(x)\rangle$, and reference density, $\phi(\mathbf{r})=1+\varepsilon \cos \left(q_{x} x\right)$ with $\varepsilon=0.2$ and $q_{x} R_{\mathrm{eo}}=\pi$ and $2 \pi$, respectively. (b) Reduced chemical potential, $\mu^{*} \equiv \frac{\mu R_{\mathrm{eo}}^{3}}{k_{B} T \sqrt{\overline{\mathcal{N}}}}$ of the restraint homopolymer system according to (55). The analytic approximations, (58) and (59), are presented for comparison as solid and dashed lines, respectively. (c) Force density $\left\langle K_{x}(x)\right\rangle R_{\mathrm{eo}} / k_{B} T$ and gradient of the chemical potential of a gas of segments as indicated by solid lines. (d) Gradient of the reduced chemical potential of the restraint homopolymer system according to (51). The analytic prediction, (58), is plotted as a solid line

which restores non-linear terms that correspond to the combinatorial entropy of the macromolecules but retains the linear approximation in the Laplace term and additionally requires $\left(q R_{\mathrm{eo}}\right)^{2} \rightarrow 0$.

In panel (c) of Fig. 1 we present the density of bonded forces, $\left\langle K_{x}\right\rangle$, and compare the simulation results with the gradient of the combinatorial entropy of the gas of non-bonded segments, $k_{B} T \nabla \ln \phi$. The difference between the two data sets is proportional to $\nabla \mu$ according to (51).

The resulting $\nabla \mu$ is presented in panel (d), which also depicts the analytic prediction, (58). For $q_{x} R_{\mathrm{eo}}=\pi$ the data are consistent with the results from panel (b) and the RPA prediction. For the variation with the larger wave vector, $q_{x} R_{\mathrm{eo}}=2 \pi$, some of the deviations between the simulation results and the RPA prediction as well as some of the discrepancies between the results obtained by field-theoretic umbrella sampling and the force-matching technique are related to coarse spatial resolution, $\Delta L=R_{\mathrm{eo}} / 6$ or $q_{x} \Delta L \approx 1.05$, with which the density profiles are resolved.

Second, we consider a symmetric copolymer system with chain discretizations $N=32$, $\kappa_{\mathrm{o}} N=0, \chi_{\mathrm{o}} N=0$, and $\lambda N=100$. We again restrain the system to a spatial variation of the 

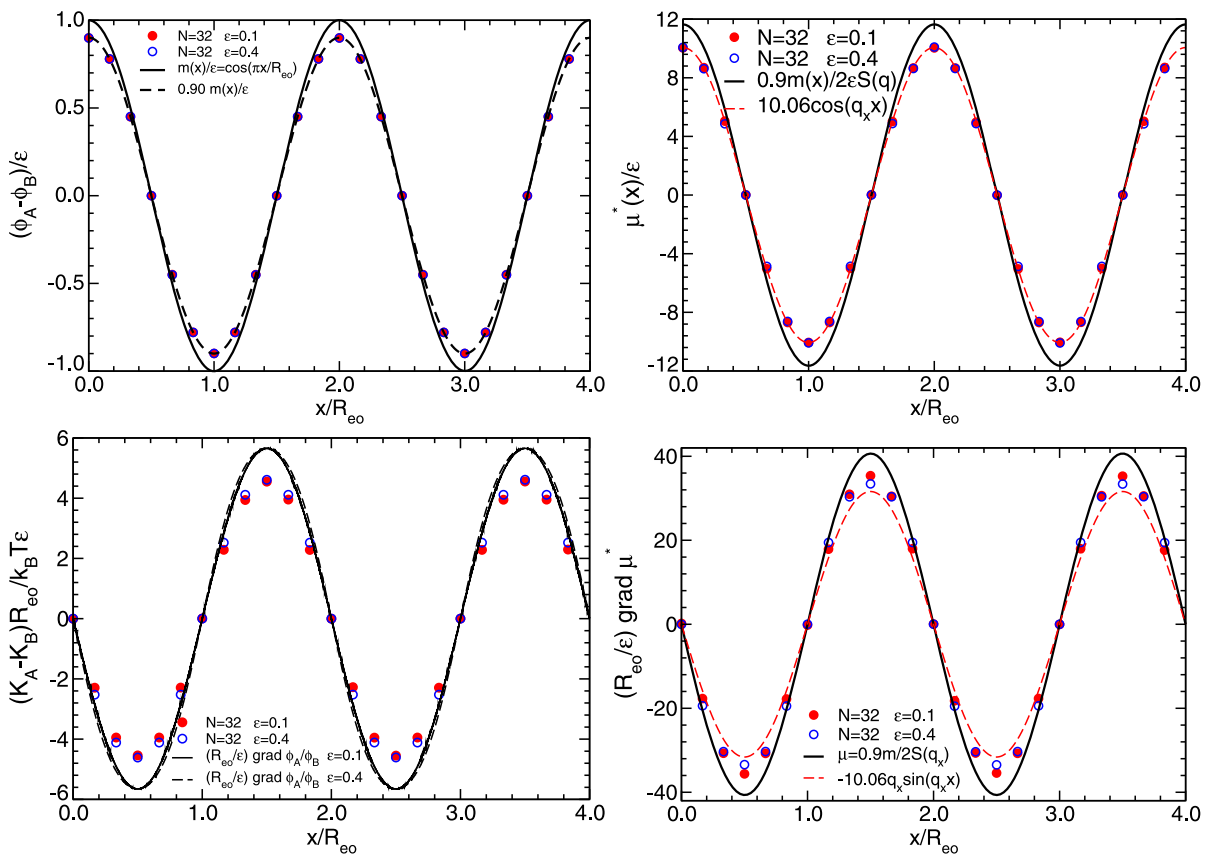

Fig. 2 Restraint simulation of a symmetric copolymer system with $N=32, \kappa_{\mathrm{o}} N=0, \chi_{\mathrm{o}} N=0, \overline{\mathcal{N}}=128^{2}$, $\lambda N=100$, and zeroth-order assignment (cf. (68)) using $\Delta L=R_{\mathrm{eo}} / 6$. (a) One-dimensional composition profile, $\left\langle\hat{\phi}_{A}(x)\right\rangle-\left\langle\hat{\phi}_{B}(x)\right\rangle$, and reference order parameter, $m(\mathbf{r})=\phi_{A}(\mathbf{r})-\phi_{B}(\mathbf{r})=\varepsilon \cos \left(q_{x} x\right)$ with $\varepsilon=0.1$ and 0.4 , and $q_{x} R_{\mathrm{eo}}=\pi$. (b) Reduced chemical potential, $\mu^{*} \equiv \frac{\left[\mu_{A}-\mu_{B}\right] R_{\mathrm{eo}}^{3}}{k_{B} T \sqrt{\overline{\mathcal{N}}}}$ of the restraint copolymer system according to (55). The analytic approximation, (34), is presented for comparison as solid line. A fit of the simulation results is indicated by the dashed line. (c) $x$-component of the force density $\left\langle K_{A}(x)-K_{B}(x)\right\rangle R_{\mathrm{eo}} / k_{B} T$ and gradient of the chemical potential of an ideal mixture of segments as indicated by solid lines. (d) Reduced chemical potential of the restraint copolymer system according to (51). The analytic prediction, (34), is plotted as a solid line and the gradient of the fit from panel (b) is shown as a dashed line

composition that is characterized by a single wave vector

$$
\phi_{A}(\mathbf{r})=1-\phi_{B}(\mathbf{r})=\frac{1}{2}\left[1+\varepsilon \cos \left(\frac{\pi x}{R_{\mathrm{eo}}}\right)\right]
$$

Two amplitudes are employed $\varepsilon=0.1$ and 0.4 . The composition profile of the constraint copolymer system is depicted in Fig. 2(a). Since composition fluctuations in an ideal gas of copolymers cost more free energy than density fluctuations in homopolymers at small and intermediate wave vectors, the same strength of the restraining potential has a weaker effect in the copolymer system than in a homopolymer system, i.e., the difference between the imposed order-parameter variation, $\phi_{A}-\phi_{B}$, and the observed composition, $\left\langle\hat{\phi}_{A}\right\rangle-\left\langle\hat{\phi}_{B}\right\rangle$, remains larger than in Fig. 1(a).

In Fig. 2(b) we present the chemical exchange potential, $\mu_{A}(\mathbf{r})-\mu_{B}(\mathbf{r})$, and compare it with the prediction of the RPA, (34). In agreement with the RPA, the simulation data linearly depend on the amplitude, $\varepsilon$, of the order-parameter variation. The magnitude of the chemical potential variation is slightly overestimated by the RPA. Panel (c) depicts the difference of the forces that act on $A$ segments and $B$ segments and compares the results 
with the combinatorial contribution, $k_{B} T \nabla \ln \phi_{A} / \phi_{B}$, that corresponds to an ideal mixture of non-bonded segments. From these simulation data we estimate the chemical potential via (51). The RPA prediction also slightly overestimates the variation of the gradient of the chemical potential and the simulation data obtained with the force-matching technique are compatible with the results obtained from field-theoretic umbrella sampling (cf. panel (b)).

\subsection{Top-Down Approach and Universality}

While the two strategies of extracting the excess free-energy functional from a microscopic description are of fundamental interest and, additionally, may become useful for relating a coarse-grained particle-based model to a continuum description in a concurrent coupling scheme [21], a systematic Ansatz-free derivation of the excess free-energy functional from a chemically realistic model on the atomistic scale remains a formidable task. The reason for this difficulty can be traced back to the scale separation between the monomeric repeat units on the atomistic scale and the coarse-grained segments that are comprised of many monomeric repeat units. As mentioned before, the typical energy scale on the atomistic scale is electron Volts $\approx 40 k_{B} T$, while the relevant free-energy scale associated with a bond between coarse-grained segments or their non-bonded interaction is on the order of $k_{B} T$ or $10^{-2} k_{B} T$, respectively. It is the latter scale that dictates the self-assembly. Neither are the interactions on the microscopic scale know with a sufficient accuracy nor exist sufficiently accurate systematic coarse-graining procedures that can preserve the subtle free-energy differences across the scales.

Fortunately, the same separation between the time, length and energy scales of the monomeric repeat units and the coarse-grained segments imparts a great degree of universality onto the structure and thermodynamics of dense multicomponent polymer systems. Therefore, a physically motivated top-down approach, where we construct a minimal model that only incorporates the relevant interactions that are necessary to bring about the physics of self-assembly in amphiphilic systems, is an alternative to systematic bottom-up approaches in particular for dense polymer systems $[4,6]$. From the very definition, a model of amphiphiles has to include the connectivity of the two opposing building blocks and their mutual repulsion. In the following, we use the bead-spring Hamiltonian, (2), to model the connectivity along the molecular backbone. The experimentally accessible value of the end-to-end distance, $R_{\text {eo }}$, can be used to relate the model to a specific experimental system.

The non-bonded interactions, $\mathcal{H}_{\mathrm{nb}}$, describe the repulsion between unlike species, which gives rise to self-assembly. Moreover, the effect of the harsh excluded volume interaction that operate between the monomeric repeat units is to limit local fluctuations of the density. Since the typical length scale of our coarse-grained model is set by a fraction of $R_{\mathrm{eo}}$, it is not necessary to enforce incompressibility on the atomistic length scale but it suffices to suppress density fluctuations down to a small fraction of $R_{\text {eo }}$. These two relevant properties of the non-bonded interactions can be modeled by a minimal excess free-energy functional of the form:

$$
\begin{aligned}
\mathcal{F}_{\mathrm{nb}} & =\mathcal{F}_{\text {melt }}+\mathcal{F}_{\text {ord }} \quad \text { with } \\
\frac{\mathcal{F}_{\text {melt }}\left[\phi_{A}, \phi_{B}\right]}{k_{B} T \sqrt{\overline{\mathcal{N}}}} & =\frac{\kappa_{\mathrm{o}} N}{2} \int \frac{\mathrm{d}^{3} \mathbf{r}}{R_{\text {eo }}^{3}}\left[\phi_{A}(\mathbf{r})+\phi_{B}(\mathbf{r})-1\right]^{2} \\
\frac{\mathcal{F}_{\text {ord }}\left[\phi_{A}, \phi_{B}\right]}{k_{B} T \sqrt{\overline{\mathcal{N}}}} & =-\frac{\chi_{\mathrm{o}} N}{4} \int \frac{\mathrm{d}^{3} \mathbf{r}}{R_{\text {eo }}^{3}}\left[\phi_{A}(\mathbf{r})-\phi_{B}(\mathbf{r})\right]^{2}
\end{aligned}
$$


The first contribution can be conceived as the expansion of the equation of state of the polymer material around the reference segment density, $\rho_{\mathrm{o}}$, and it has been introduced by Helfand [120]. The second contribution describes the regular mixing of a two-component system and it has first been employed to model the thermodynamics of polymer mixtures by Huggins and Flory [79, 80]. This minimal description of the non-bonded interactions is characterized by the coarse-grained parameters, $\chi_{\mathrm{o}} N, \kappa_{\mathrm{o}} N$, and $\overline{\mathcal{N}}$. Note that these parameters will remain invariant, if we choose a different discretization, $N$, of the molecular contour. $\overline{\mathcal{N}}$ quantifies the number of neighbors with which a reference molecule interacts. $\chi_{\mathrm{o}} N$ describes the strength of the repulsion between the block of the copolymer molecule, which can be measured, e.g., by scattering experiments. $\kappa_{\mathrm{o}} N$ is related to the inverse compressibility of the dense polymer liquid.

The strength of these interactions and the entropic cost of a spatially inhomogeneous density distribution result in characteristic length scales. Within the mean-field approximation, one obtains for long flexible polymers (ground state approximation) [120, 121]:

$$
\frac{w}{R_{\mathrm{eo}}} \approx \frac{1}{\sqrt{6 \chi_{\mathrm{o}} N}} \quad \text { and } \quad \frac{\xi}{R_{\mathrm{eo}}} \approx \frac{1}{\sqrt{12 \kappa_{\mathrm{o}} N}} \quad(\mathrm{GSA})
$$

$w$ characterizes the intrinsic width of an interface between $A$ and $B$ domains, while $\xi$ quantifies the correlation length of density fluctuations. These are the two smallest length scales of the model. The contour discretization $b=R_{\mathrm{eo}} / \sqrt{N}$ and any spatial discretization (see below) must be smaller than these two length scales in order to observe universal properties that do not depend on these discretization parameters. This minimal model for the non-bonded interaction is also the standard model of a field-theoretic description [120, 122] (e.g., the self-consistent field theory), and it has been fruitfully applied to a large variety of phenomena in dense multi-component polymer systems.

One particular advantage of this top-down approach consists in the simple physical interpretation that the coarse-grained parameters adopt in limiting cases (e.g., within the meanfield approximation). Moreover, the functional form of the excess free-energy functional of non-bonded interactions can benefit from the vast knowledge accumulated by studies of fluids within classical density functional theory [11,22]. Many sophisticated density functional approaches have been devised to describe the structure and thermodynamics of simple and complex fluids as well as crystals [123, 124]. Even if we disregard the molecular connectivity for a moment, however, there is a fundamental difference between the excess free-energy functional, $\mathcal{F}_{\mathrm{nb}}$ defined by (7), and a classical density functional, $F_{\mathrm{DFT}}\left[\phi_{A}, \phi_{B}\right]$ for the liquid of non-bonded coarse-grained segments. In our soft coarse-grained model, the partition function is obtained from (7) via integrating over the fluctuations of the densities, $\phi_{A}$ and $\phi_{B}$, while, in classical density functional theory, the partition function is obtained by the saddle point of the classical density functional.

$$
Z=\min _{\phi_{A}, \phi_{B}} F_{\mathrm{DFT}}\left[\phi_{A}, \phi_{B}\right]
$$

Nevertheless, one can expect that the functional forms of the excess free-energy functional and the classical density functional for the liquid of non-bonded segments is similar, and short-ranged fluctuations merely lead to a renormalization of the coarse-grained parameters $[60,62,63]$.

Inspired by this relation to classical density functional theory, various generalizations of this minimal model have been explored to describe more complex situations. For instance, a third-order virial expansion has been employed to describe the competition between liquidvapor and liquid-liquid separation. This excess free-energy functional has found application 
to model bubble nucleation in polymer-solvent mixtures [94], to devise a solvent-free description of lipid membranes [11], and to study the structure of mixed brushes and copolymer brushes [95, 125, 126]. Instead of a local excess free-energy functional, one can use a weighted density functional [127] in order to incorporate some liquid-like packing into the fluid of coarse-grained segments. This approach has been followed to construct a solventfree model for lipid membranes that exhibits a liquid-gel transition [22].

\subsection{Non-bonded Interactions: Collocation Grid Versus Weighting Function}

The schematic definition of the microscopic density, (4), does not lend itself to particle-based computer simulations and different models can be distinguished by the way the $\delta$-function is regularized. Two different strategies have been devised-grid-based descriptions $[15,25]$ and weighting functions [29, 97] — and we discuss them in turn.

Grid-based methods have attracted abiding interesting for calculating electrostatic interactions [128] or in form of particle-in-cell techniques in plasma physics [129]. In the grid-based scheme, one discretizes space in cubic cells of linear dimension, $\Delta L$. Each cell is identified by its index, c. We define the local microscopic densities, $\phi_{A}(\mathbf{c})$ and $\phi_{B}(\mathbf{c})$, for each cell $\mathbf{c}$ of this collocation grid by assigning particle positions to the grid cells according to [15]

$$
\hat{\phi}_{A}(\mathbf{c})=\int \frac{\mathrm{d}^{3} \mathbf{r}}{\Delta L^{3}} \Pi(\mathbf{c}, \mathbf{r}) \hat{\phi}_{A}(\mathbf{r})=\frac{1}{\rho_{\mathrm{o}} \Delta L^{3}} \sum_{i=1}^{n} \sum_{s=1}^{N} \gamma_{i, s} \Pi\left(\mathbf{c}, \mathbf{r}_{i, s}\right)
$$

The assignment function is normalized

$$
\sum_{\mathbf{c}} \Pi(\mathbf{c}, \mathbf{r})=1 \quad \forall \mathbf{r} \quad \text { and } \quad \int \mathrm{d}^{3} \mathbf{r} \Pi(\mathbf{c}, \mathbf{r})=\Delta L^{3} \quad \forall \mathbf{c}
$$

i.e., the contribution of a particle to all cell adds up to unity irrespectively of its position and the volume assigned to each grid cell is $\Delta L^{3} . \Delta L$ plays a similar role as the range of non-bonded interactions in an off-lattice description. $\Delta L$ must be smaller than all physical length scales for the model properties be independent from the spatial discretization. The discretization of the molecular architecture gives rise to another microscopic length scale, the statistical segment length $b=R_{\text {eo }} / \sqrt{N}$. Typically, one chooses both length scales to be of the same order, i.e. $\Delta L \approx R_{\mathrm{eo}} / \sqrt{N}$.

The zeroth-order assignment function, $\Pi_{(0)}$, associates a particle at position $\mathbf{r}$ with the nearest grid point $\mathbf{c}$, i.e.,

$$
\Pi_{(0)}(\mathbf{c}, \mathbf{r})= \begin{cases}1 & \text { if }\left|\mathbf{r}_{\alpha}-\mathbf{c}_{\alpha}\right|<\frac{\Delta L}{2} \forall \alpha \in\{x, y, z\} \\ 0 & \text { otherwise }\end{cases}
$$

where $\mathbf{r}_{\alpha}$ and $\mathbf{c}_{\alpha}$ denote the Cartesian coordinates of the particle and the grid point.

The advantage of this assignment function is its computational simplicity and the irrelevance of self-interactions (see below). The disadvantage consists in the discontinuous change of the grid-based density when a particle straddles a cell boundary. For instance, this choice gives rise to a discontinuous non-bonded force, $-\partial \mathcal{H}_{\mathrm{nb}}\left(\left\{\mathbf{r}_{i, s}\right\}\right) / \partial \mathbf{r}_{j, t}$ that acts on the $t^{\text {th }}$ segment of polymer $j$.

The first-order assignment function, which is defined by [17, 130]

$$
\Pi_{(1)}(\mathbf{c}, \mathbf{r})=\prod_{\alpha \in\{x, y, z\}} \pi\left(\left|\mathbf{r}_{\alpha}-\mathbf{c}_{\alpha}\right|\right) \quad \text { with } \pi(d)= \begin{cases}1-\frac{|d|}{\Delta L} & \text { for }|d| \leq \Delta L \\ 0 & \text { otherwise }\end{cases}
$$


can overcome this disadvantage.

Using this grid-based assignment, we can rewrite the excess free energy of non-bonded interactions in terms of particle interactions. Typical terms in $\mathcal{H}_{\mathrm{nb}}$ are quadratic in the microscopic densities, and we can rewritten them in the following grid-based form [25]:

$$
\begin{aligned}
& \sqrt{\overline{\mathcal{N}}} \int \frac{\mathrm{d}^{3} \mathbf{r}}{R_{\mathrm{eo}}^{3}} \hat{\phi}_{A}(\mathbf{r}) \hat{\phi}_{B}(\mathbf{r}) \\
& \equiv \sqrt{\overline{\mathcal{N}}} \frac{\Delta L^{3}}{R_{\mathrm{eo}}^{3}} \sum_{\mathbf{c}}\left(\frac{1}{\rho_{\mathrm{o}} \Delta L^{3}} \sum_{i, s} \gamma_{i, s} \Pi\left(\mathbf{c}, \mathbf{r}_{i, s}\right)\right)\left(\frac{1}{\rho_{\mathrm{o}} \Delta L^{3}} \sum_{j, t}\left(1-\gamma_{j, t}\right) \Pi\left(\mathbf{c}, \mathbf{r}_{j, t}\right)\right) \\
& =\frac{N}{\rho_{\mathrm{o}} \Delta L^{3}} \frac{1}{N^{2}} \sum_{(i, s)(j, t)} \gamma_{i, s}\left(1-\gamma_{j, t}\right) \sum_{\mathbf{c}} \Pi\left(\mathbf{c}, \mathbf{r}_{i, s}\right) \Pi\left(\mathbf{c}, \mathbf{r}_{j, t}\right) \\
& =\frac{1}{N^{2}} \sum_{(i, s)(j, t)} \gamma_{i, s}\left(1-\gamma_{j, t}\right) v\left(\mathbf{r}_{i, s}, \mathbf{r}_{j, t}\right)
\end{aligned}
$$

Thus a quadratic excess free energy in conjunction with evaluating the local densities via a collocation grid corresponds to pairwise interactions [25]

$$
v\left(\mathbf{r}_{i, s}, \mathbf{r}_{j, t}\right)=\frac{1}{\sqrt{\overline{\mathcal{N}}}} \frac{R_{\mathrm{eo}}^{3}}{\Delta L^{3}} \sum_{\mathbf{c}} \Pi\left(\mathbf{c}, \mathbf{r}_{i, s}\right) \Pi\left(\mathbf{c}, \mathbf{r}_{j, t}\right)
$$

Higher powers of the densities (e.g., cubic terms) in the excess free-energy functional result in higher-order interactions (e.g., three-body interactions). The dependence of this pairwise interaction on density, $\frac{1}{\sqrt{\overline{\mathcal{N}}}} \sim \frac{1}{\rho_{0}}$, guarantees that the high-density limit, $\overline{\mathcal{N}} \rightarrow \infty$, is well defined.

While the molecular conformations live in continuum space, the interactions make reference to the underlying collocation grid; the interactions do not only depend on the difference between the segment positions but also on their relative position to the collocation grid. Therefore, they are not translationally invariant and not isotropic. As a consequence, one cannot use the virial expression to compute the contribution of the non-bonded interactions to the pressure but special simulation techniques for lattice models have to be employed [83, 131, 132].

We also point out that the so-defined excess free energy contains the interaction of a coarse-grained segment with itself because the quadratic term proportional to $\frac{1}{2} \hat{\phi}_{A}^{2}$ can be split into interactions between pairs of $A$ segments and an additional self-interaction term.

$$
\begin{aligned}
& \frac{\sqrt{\overline{\mathcal{N}}}}{2} \int \frac{\mathrm{d}^{3} \mathbf{r}}{R_{\mathrm{eo}}^{3}} \hat{\phi}_{A}(\mathbf{r}) \hat{\phi}_{A}(\mathbf{r}) \\
& =\frac{1}{2 N^{2}} \sum_{(i, s)(j, t)} \gamma_{i, s} \gamma_{j, t} v\left(\mathbf{r}_{i, s}, \mathbf{r}_{j, t}\right) \\
& =\frac{1}{N^{2}} \sum_{[(i, s)(j, t)]_{A A}} v\left(\mathbf{r}_{i, s}, \mathbf{r}_{j, t}\right)+\frac{1}{2 N^{2}} \sum_{i, s} \gamma_{i, s} v\left(\mathbf{r}_{i, s}, \mathbf{r}_{i, s}\right)
\end{aligned}
$$

where $[(i, s)(j, t)]_{A A}$ denotes all pairs of $A$ segments. With these considerations the gridbased non-bonded interactions take the form 


$$
\begin{aligned}
\frac{\mathcal{H}_{\mathrm{nb}}\left(\left\{\mathbf{r}_{i, s}\right\}\right)}{k_{B} T}= & +\frac{1}{N^{2}} \sum_{[(i, s)(j, t)]_{A A}}\left(\kappa_{\mathrm{o}} N-\frac{\chi_{\mathrm{o}} N}{2}\right) v\left(\mathbf{r}_{i, s}, \mathbf{r}_{j, t}\right) \\
& +\frac{1}{N^{2}} \sum_{[(i, s)(j, t)]_{B B}}\left(\kappa_{\mathrm{o}} N-\frac{\chi_{\mathrm{o}} N}{2}\right) v\left(\mathbf{r}_{i, s}, \mathbf{r}_{j, t}\right) \\
& +\frac{1}{N^{2}} \sum_{[(i, s)(j, t)]_{A B}}\left(\kappa_{\mathrm{o}} N+\frac{\chi_{\mathrm{o}} N}{2}\right) v\left(\mathbf{r}_{i, s}, \mathbf{r}_{j, t}\right) \\
& +\frac{\mathcal{H}_{\mathrm{self}}\left(\left\{\mathbf{r}_{i, s}\right\}\right)}{k_{B} T} \text { with } \\
\frac{\mathcal{H}_{\mathrm{self}}\left(\left\{\mathbf{r}_{i, s}\right\}\right)}{k_{B} T}= & \frac{\kappa_{\mathrm{o}} N V \sqrt{\overline{\mathcal{N}}}}{2 R_{\mathrm{eo}}^{3}}+\frac{1}{2 N^{2}} \sum_{i, s}\left(\kappa_{\mathrm{o}} N-\frac{\chi_{\mathrm{o}} N}{2}\right) v\left(\mathbf{r}_{i, s}, \mathbf{r}_{i, s}\right)
\end{aligned}
$$

If one employs the zeroth-order assignment, $\Pi_{(0)}$, the self-interactions will be immaterial because $v\left(\mathbf{r}_{i, s}, \mathbf{r}_{i, s}\right)=\frac{N}{\rho_{0} \Delta L^{3}}$ is independent from the segment position $\mathbf{r}_{i, s}$. Thus the selfenergy is merely a constant energy off-set that does not alter the statistical weight of the different configurations. This is no longer true for higher-order assignment functions. For instance, for the first-order assignment function, (69), one obtains:

$$
v_{(1)}\left(\mathbf{r}_{i, s}, \mathbf{r}_{i, s}\right)=\frac{N}{\rho_{\mathrm{o}} \Delta L^{3}} \sum_{\mathbf{c}} \prod_{\alpha} \pi^{2}\left(\left|\mathbf{r}_{\alpha}-\mathbf{c}_{\alpha}\right|\right)
$$

Thus the self-interaction takes the explicit form

$$
\frac{\mathcal{H}_{(1) \operatorname{self}}\left(\left\{\mathbf{r}_{i, s}\right\}\right)}{k_{B} T}=\frac{\kappa_{\mathrm{o}} N V \sqrt{\overline{\mathcal{N}}}}{2 R_{\mathrm{eo}}^{3}}+\frac{\kappa_{\mathrm{o}}-\frac{\chi_{\mathrm{o}}}{2}}{2 \rho_{\mathrm{o}} \Delta L^{3}} \sum_{i, s} \sum_{\mathbf{c} \in C\left(\mathbf{r}_{i, s}\right)} \prod_{\alpha}\left(1-\frac{\mid \mathbf{r}_{\alpha}-\mathbf{c}_{\alpha \mid}}{\Delta L}\right)^{2}
$$

where the sum over grid points is extended over the eight corners of the cube $C\left(\mathbf{r}_{i, s}\right)$, which contains the position of the segment. If one does not compensate for these self-interactions, the segment density will exhibit spurious oscillations within a cell of the collocation grid. These effects have previously been observed [17]. If one subtracts the self-interactions explicitly from the non-bonded interactions, the density variation within a grid cell can be significantly reduced as shown in Fig. 3. Correcting for the self-interactions, however, we cannot expect to eliminate density variations within a grid cell completely because the pairwise interactions remain not translationally invariant.

Instead of assigning the microscopic densities to a collocation grid, one can represent the $\delta$-function in (4) as a limit of a weighting function, $\omega$. This strategy has been introduced by Zuckermann and co-workers [97, 133]. We define a weighted density according to

$$
\hat{\phi}_{A, \omega}(\mathbf{r})=\int \frac{\mathrm{d}^{3} \mathbf{r}^{\prime}}{\Delta L^{3}} \omega\left(\left|\mathbf{r}-\mathbf{r}^{\prime}\right|\right) \hat{\phi}_{A}\left(\mathbf{r}^{\prime}\right)=\frac{1}{\rho_{\mathrm{o}} \Delta L^{3}} \sum_{i, s} \gamma_{i, s} \omega\left(\left|\mathbf{r}-\mathbf{r}_{i, s}\right|\right)
$$

The function $\omega(|\mathbf{r}|)$ is normalized as follows

$$
\int \mathrm{d}^{3} \mathbf{r} \omega(|\mathbf{r}|)=\Delta L^{3}
$$

i.e., $\Delta L^{3}$ characterizes the volume, over which the original $\delta$-function in (4) is smeared out. 


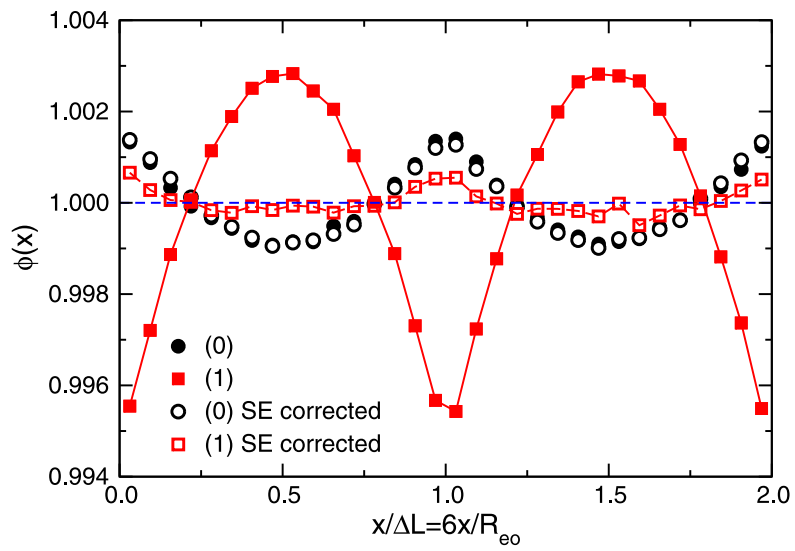

Fig. 3 Effect of self-interactions on the density profiles for a polymer melt with invariant degree of polymerization $\overline{\mathcal{N}}=128^{2}$, systems size $L=4 R_{\mathrm{eo}}$, and interaction parameters $\kappa_{\mathrm{O}} N=50$ and $\chi_{\mathrm{o}} N=0$. The contour of the molecules is discretized into $N=32$ segments and the spatial discretization is $\Delta L=R_{\mathrm{eo}} / 6$. The figure presents one-dimensional profiles of the total segment density, $\phi(x)$, using the zeroth-order assignment (0) (cf. (68)), the first-order assignment (1) (cf. (69)) with and without the self-interaction correction, (76), respectively. There is no effect of self-interactions on the zeroth-order assignment. For the first-order assignment without self-energy corrections, spurious density oscillations with a relative amplitude of $5 \cdot 10^{-3}$ are observed on the length scale below the grid spacing. Subtracting the self-interactions, we can reduce the influence of the underlying grid and the density is smoother than for the zeroth-order assignment

In this case, quadratic terms in the excess free energy also correspond to densitydependent pairwise potentials

$$
\begin{aligned}
& \sqrt{\overline{\mathcal{N}}} \int \frac{\mathrm{d}^{3} \mathbf{r}}{R_{\mathrm{eo}}^{3}} \hat{\phi}_{A}(\mathbf{r}) \hat{\phi}_{B}(\mathbf{r}) \\
& \equiv \sqrt{\overline{\mathcal{N}}} \int \frac{\mathrm{d}^{3} \mathbf{r}}{R_{\mathrm{eo}}^{3}}\left(\frac{1}{\rho_{\mathrm{o}} \Delta L^{3}} \sum_{i, s} \gamma_{i, s} \omega\left(\left|\mathbf{r}-\mathbf{r}_{i, s}\right|\right)\right)\left(\frac{1}{\rho_{\mathrm{o}} \Delta L^{3}} \sum_{j, t}\left(1-\gamma_{j, t}\right) \omega\left(\left|\mathbf{r}-\mathbf{r}_{j, t}\right|\right)\right) \\
& =\frac{N}{\rho_{\mathrm{o}} \Delta L^{3}} \frac{1}{N^{2}} \sum_{(i, s)(j, t)} \gamma_{i, s}\left(1-\gamma_{j, t}\right) \int \frac{\mathrm{d}^{3} \mathbf{r}}{\Delta L^{3}} \omega\left(\left|\mathbf{r}-\mathbf{r}_{i, s}\right|\right) \omega\left(\left|\mathbf{r}-\mathbf{r}_{j, t}\right|\right) \\
& =\frac{1}{N^{2}} \sum_{(i, s)(j, t)} \gamma_{i, s}\left(1-\gamma_{j, t}\right) v\left(\left|\mathbf{r}_{i, s}-\mathbf{r}_{j, t}\right|\right)
\end{aligned}
$$

which are, however, translationally invariant and isotropic

$$
v\left(\left|\mathbf{r}_{i, s}-\mathbf{r}_{j, t}\right|\right)=\frac{N}{\rho_{\mathrm{o}} \Delta L^{3}} \int \frac{\mathrm{d}^{3} \mathbf{r}}{\Delta L^{3}} \omega\left(\left|\mathbf{r}-\mathbf{r}_{i, s}\right|\right) \omega\left(\left|\mathbf{r}-\mathbf{r}_{j, t}\right|\right)
$$

The normalization of the weighting function determines the integrated strength of the pairwise interactions

$$
\int \frac{\mathrm{d}^{3} \mathbf{r}}{R_{\mathrm{eo}}^{3}} v(|\mathbf{r}|)=\frac{1}{\sqrt{\overline{\mathcal{N}}}}
$$

where the $\overline{\mathcal{N}}$-dependence guarantees that the limit of high density remains well defined. 
Zuckermann el al. [97, 133] used a Gaussian weighting function

$$
\omega(|\mathbf{r}|)=\left(\frac{3}{2 \pi}\right)^{3 / 2}\left(\frac{\Delta L}{\sigma}\right)^{3} \exp \left(-\frac{3 \mathbf{r}^{2}}{2 \sigma^{2}}\right)
$$

and this expression also results in a Gaussian form of the pairwise interactions

$$
\sqrt{\overline{\mathcal{N}}} v(|\mathbf{r}|)=\left(\frac{3}{4 \pi}\right)^{3 / 2}\left(\frac{R_{\mathrm{eo}}}{\sigma}\right)^{3} \exp \left(-\frac{3 \mathbf{r}^{2}}{4 \sigma^{2}}\right)
$$

Computationally it is more convenient to use a weighting function of finite support because it results in pairwise interactions with a finite range. A zeroth-order weighting function is the characteristic function of a sphere with radius $\sigma=(3 / 4 \pi)^{1 / 3} \Delta L$.

$$
\omega(|\mathbf{r}|)= \begin{cases}1 & \text { for }|\mathbf{r}| \leq \sigma=\sqrt[3]{\frac{3}{4 \pi}} \Delta L \\ 0 & \text { otherwise }\end{cases}
$$

In this case, the pairwise potential stems from the lens-shaped overlap of the two spheres around each segment,

$$
\sqrt{\overline{\mathcal{N}}} v(|\mathbf{r}|)= \begin{cases}\frac{3}{8 \pi}\left(\frac{R_{\mathrm{e} o}}{\sigma}\right)^{3}\left(2+\frac{|\mathbf{r}|}{2 \sigma}\right)\left(1-\frac{|\mathbf{r}|}{2 \sigma}\right)^{2} & \text { for }|\mathbf{r}| \leq 2 \sigma \\ 0 & \text { otherwise }\end{cases}
$$

The choice of the weighting function is largely dictated by computational convenience. In the case of large $\overline{\mathcal{N}}$, however, an inappropriate choice of the weighting function may lead to the formation of cluster crystals [134]. These are periodic lattices formed by particles with a soft potential that allows the particles to overlap. The lattice spacing is independent of density and, upon increasing the density, one observes that one unit cell of the lattice is occupied by multiple particles. Within the mean-spherical-approximation (MSA), Likos et al. [134] demonstrate that a homogeneous liquid will become unstable with respect to the formation of these cluster crystal at high density, if the Fourier transform, $V(q)$, of the pair-potential becomes negative for some wave vectors. The wave vector of the minimum of $V(q)$ dictates the lattice spacing.

If we consider a one-component system of non-bonded segments, the energy of the coarse-grained model can be written in the form

$$
\begin{aligned}
& \mathcal{H}_{\mathrm{nb}}\left(\left\{\mathbf{r}_{i, s}\right\}\right)=k_{B} T \sqrt{\overline{\mathcal{N}}} \frac{\kappa_{\mathrm{o}} N}{2} \int \frac{\mathrm{d}^{3} \mathbf{r}}{R_{\mathrm{eo}}^{3}} \hat{\phi}_{A}^{2}=\sum_{[(i, s)(j, t)]_{A A}} V\left(\left|\mathbf{r}_{i, s}-\mathbf{r}_{j, t}\right|\right) \\
& \text { with } \quad V(r)=\frac{k_{B} T \kappa_{\mathrm{o}} N}{N^{2} \sqrt{\overline{\mathcal{N}}}}\left(\frac{R_{\mathrm{eo}}}{\sigma}\right)^{3} \Phi\left(\frac{r}{\sigma}\right)=\frac{k_{B} T \kappa_{\mathrm{o}}}{\rho_{\mathrm{o}} \sigma^{3}} \Phi\left(\frac{r}{\sigma}\right)
\end{aligned}
$$

where $\Phi(r / \sigma) \equiv \sqrt{\overline{\mathcal{N}}}\left(\sigma / R_{\mathrm{eo}}\right)^{3} v(r)$ characterizes the shape of the pairwise potential. The stability condition within MSA requires [134]

$$
0<1+\frac{\rho_{\mathrm{o}}}{k_{B} T} V(q)=1+\kappa_{\mathrm{o}} \Phi(q \sigma) \quad(\mathrm{MSA})
$$

where $V(q)$ and $\Phi(q \sigma)$ are the Fourier transforms of the pairwise interaction and the shape function, respectively. 
Due to the translational invariance and isotropy of the pairwise potentials, the weightingfunction approach allows us to easily compute the pressure without resorting to special simulation techniques for lattice systems. This ability is advantageous, e.g., in order to determine the equilibrium periodicity of a self-assembled structure. It also allows for simulations at constant pressure or in the Gibbs ensemble [18, 29]. Higher-order interactions, that arise from higher powers of the density in the excess free-energy functional, can be efficiently investigated by multi-particle dissipative particle dynamics $[22,135,136]$. Moreover, a connection between the excess free-energy functional and weighted density functional theory of liquids can be established [22], which allows us to tune the liquid-like packing of the fluid of segments.

The major disadvantage of the weighting-function technique consists in the computational expense of calculating the pairwise interactions. In the grid-based approach, the energy of a segment with its surrounding can be computed from the knowledge of the gridbased density. In case of the zeroth-order assignment the densities at the nearest grid point are required; in case of the first-order assignment one needs the information about the eight corners of the cube that contains the segment. In the case of a weighting function, however, the energy involves the explicit computation of the pairwise interactions between the segment with its neighbors. This calculation can be efficiently performed via a cell list, where the cell's linear dimension is the range of the pairwise interaction, $\mathcal{O}(\Delta L)$. All interactions in the 27 cells around the one that contains the segment have to be considered. Each cell, in turn, is comprised of $\rho_{\mathrm{o}} \Delta L^{3}$ segments. Altogether $27 \rho_{\mathrm{o}} \Delta L^{3}=27 N \sqrt{\overline{\mathcal{N}}}\left(\Delta L / R_{\mathrm{eo}}\right)^{3}$ pairwise interactions have to be considered. For a typical choice of parameters (cf. Sect. 2.2), $N=31, \overline{\mathcal{N}}=10^{4}, \Delta L / R_{\mathrm{eo}}=1 / 6$ this amounts to $3 \cdot 10^{2}$ interaction pairs. Thus the gridbased technique offers a significant computational advantage for dense polymer systems.

\subsection{Other Soft Coarse-Grained Models}

The minimal excess free-energy functional, (62) and (63), is quadratic in the densities and, in conjunction with a weighting function, gives rise to a soft coarse-grained model with isotropic and translationally invariant pairwise interactions. In typical simulations we use $\overline{\mathcal{N}}=16384$, which is characteristic for experimental polymer systems, $N=32, \Delta L=$ $R_{\mathrm{eo}} / 6$, and $\kappa_{\mathrm{o}} N=50$. Using the weighting function (84) the repulsive energy of two perfectly overlapping segments is $V(0)=\frac{3}{4 \pi} \frac{k_{B} T \kappa_{\mathrm{o}} N}{N^{2} \sqrt{\bar{N}}} \frac{R_{\mathrm{eo}}^{3}}{\sigma^{3}}<0.1 k_{B} T$, i.e., the interaction between the coarse-grained segments is very soft. Therefore, the fluid of coarse-grained segments does not exhibit significant liquid-like packing and the bare parameters of the model, $\chi_{\mathrm{o}}$ and $\kappa_{\mathrm{o}}$, are only weakly renormalized by short-ranged fluctuations or liquid-like packing effects.

Our off-lattice models shares many qualitative similarities to the coarse-grained model introduced by Groot and Warren in 1997 [12-14], which has been employed to study interfaces in homopolymer blends and the self-assembly of block copolymers. In this model, segments of type $\alpha$ and $\beta$ interact via a pairwise repulsion of the form

$$
V_{\alpha, \beta}(\mathbf{r})= \begin{cases}\frac{a_{\alpha, \beta} k_{B} T}{2}\left(1-\frac{|\mathbf{r}|}{\sigma}\right)^{2} & \text { for }|\mathbf{r}|<\sigma \\ 0 & \text { otherwise }\end{cases}
$$

where $\sigma$ sets the length scale of pairwise interactions. $2 a_{A B}>a_{A A}+a_{B B}$ in order to describe the repulsion between $A$ and $B$ segments. This potential also fulfills the condition (88). Groot and Warren typically used a segment number density of $\rho_{\mathrm{o}} \sigma^{3}=3$, a repulsive strength of the order $a_{\alpha, \beta}=25$, and $N=10$ segments per polymer. $R_{\mathrm{eo}} \approx 3 \sigma$ yields an invariant degree of polymerization of $\overline{\mathcal{N}} \approx 66$ and the energy of two perfectly overlapping 
particles is $V(0)=12.5 k_{B} T$. Both, the small value of $\overline{\mathcal{N}}$ as well as the rather large penalty for particle overlap, place the Groot-Warren model between coarse-grained models with excluded volume, e.g., a Lennard-Jones bead-spring model [84], and the soft coarse-grained model described above.

In 2009, Wittmer and co-workers have proposed a generalization of the bond fluctuation model where the overlap of segments is subjected to a finite energy penalty [76]. The authors carefully and systematically studied the influence of the soft segment-segment repulsion on the compressibility, the structure factor of density fluctuations, and the crossover from self-avoiding walk behavior to the melt behavior as the soft repulsive interactions become screened at high densities. In this work the parameters $\rho_{\mathrm{o}}=1 / 16$ and $N \leq 8192$ were employed. The statistical segment length of the bond fluctuation model is $b \approx 3.1$ resulting in large values of $\overline{\mathcal{N}}=\left(\rho_{\mathrm{o}} b^{3}\right)^{2} N \leq 28400$.

The computational advantages of soft coarse-grained models have also been highlighted by Wang in the same year [19]. In one work this author used basically the same model as described in the previous section. In a second paper [20], he employed the Hamiltonian (62) but, instead of describing the molecular conformations by an off-lattice bead-spring Hamiltonian (2), the author used random walks on a simple cubic lattice. The latter strategy speeds up the calculation of the non-bonded interactions but a finer chain discretization, $N$, is required to mimic the Gaussian chain architecture. More recently, Wang has also extended this class of models to study rod-coil block copolymer systems where orientational interactions are important [137].

\subsection{Limitations of Soft Coarse-Grained Models}

The advantages of soft coarse-grained models for dense multi-component polymer systems are chiefly based on the aggressive coarse-graining procedure-lumping a large number of monomeric repeat units of a chemically realistic representation into an effective coarsegrained segment-and the concomitant reduction of the number of degrees of freedom and the softer interactions of the coarse-grained segments. These beneficial features, however, also impart limitations. In this subsection we briefly discuss two of such limitationsmissing spatial resolution at the narrow interface between a polymer melt $[138,139]$ and a solid substrate and violation of the topological non-crossability constraint [140-147]—and indicate strategies to overcome them.

\subsubsection{Interference Between Chain Contour and Spatial Discretization and Narrow Interfaces}

Some caution will be required if the smallest discretization length scale, $b=R_{\mathrm{eo}} / \sqrt{N}$ or $\Delta L$, becomes comparable to the smallest physical length scale $[17,25,138,139]$. The narrow interfaces between a polymer melt and a solid substrate or the narrow liquid-vapor interface are prototypical examples. The intrinsic width of these interfaces is of the order of a few nanometers (or smaller) in an experimental system. In our soft coarse-grained model the intrinsic width is set by the screening length of excluded volume interaction (cf. (64)). Using typical values, $R_{\mathrm{eo}}=50 \mathrm{~nm}, \kappa_{\mathrm{o}} N=50, N=31$ and $\Delta L=R_{\mathrm{eo}} / 6$, one obtains $\xi \approx 2$ $\mathrm{nm}, b=R_{\mathrm{eo}} / \sqrt{N} \approx 9 \mathrm{~nm}$, and $\Delta L=R_{\mathrm{eo}} / 6 \approx 8 \mathrm{~nm}$. Both, $b$ and $\Delta L$, are larger than the physical length scale, $\xi$. Thus the description of the narrow interface will be affected by the discretization of the model. Choosing a finer discretization of the chain contour, $N$, and a finer discretization of space, $\Delta L$, one could, of course, overcome this limitation. This strategy, however, would also sacrifice much of the advantages of the coarse-grained model. 
Alternatively, one can ask the question, which property of the narrow polymer-solid contact is relevant for a description of the behavior on large time and length scales [139]. One important characteristics of the narrow polymer-solid contact is the surface tension. This quantity controls the stability of the polymer film with respect to de-wetting [67, 148] and, in a multi-component system, the difference between the surface tension of coexisting $A$-rich and $B$-rich domains controls the angle of $A B$ interfaces with the substrate, which inter alia influences the orientation of the self-assembled morphology with respect to the confining boundaries [68]. We suggest that the surface tension represents a coarse-grained parameter, i.e., it is the key characteristics of the surface that a coarse-grained model shall reproduce. Even if the discretization of the coarse-grained model is insufficient to resolve the structure of the narrow polymer-solid contact, one can adjust the surface tension by modifying the interaction between the liquid of coarse-grained segments and the solid. For a model with a local excess free-energy functional, $\mathcal{F}_{\text {nb }}$, it has been shown within mean-field approximation that it is even possible to choose the polymer-solid interaction to simultaneously adjust the surface tension and the density profile to the values of a description based upon continuous Gaussian threads, $N \rightarrow \infty$ [139].

\subsubsection{Crossability of Polymers}

Another feature of soft coarse-grained models is the violation of the non-crossability of polymers $[140,141]$. In models with excluded volume interactions, e.g., the Lennard-Jones bead-spring model [84], non-crossability of bonds is guaranteed by a combination of finite length of a bond and hard core diameter of a segment. Thus the crossing of bonds is automatically forbidden (or penalized by an energy that exceeds $k_{B} T$ by far). For linear polymers, non-crossability does not affect the equilibrium properties but for ring polymers is alters the scaling behavior of the chain extension with the molecular weight in a melt [150-153]. Non-crossability has, however, a rather dramatic influence on the single-chain dynamics. In experiments [154, 155] as well as in simulations [156, 157], long macromolecules in a dense melt reptate along their contour, and they can escape the tube that is formed around them by the topological interactions with neighboring polymers only at the chain ends [87]. This reptation motion leads to a self-diffusion coefficient that scales like $D \sim N^{-2}$ with chain length.

The violation of the non-crossability constraint leads to Rouse-like dynamics [158] even in a dense melt of long polymers with $D \sim N^{-1}$. The concomitant speed-up of the single chain dynamics is beneficial for equilibrating the system in computer simulation [159]. It is, however, a disadvantage for studying the kinetics of structure formation. In particular, inhomogeneous systems exhibit a coupling between the single-chain dynamics and the selfassembled morphology, because interfaces have a pronounced influence on the conformation and, in turn, the motion of individual chains [160-163].

We note, additionally, that the intermolecular correlations, which manifest themselves as the correlation hole in the intermolecular pair correlation function and corrections to the Gaussian chain statistics [69-77], also impart forces on the center of mass of a polymer. These forces result in corrections to the strictly linear time dependence of the center-of-mass mean-square displacement, $g_{3}(t)$, predicted by the Rouse model [158]. A recent analysis by Wittmer and co-workers yields the prediction [77, 78]

$$
g_{3}(t)=6 D t\left(1+\frac{c}{\sqrt{\overline{\mathcal{N}}}}\left[\frac{t}{\tau_{\mathrm{dis}}}\right]^{-1 / 4}\right)
$$

where $c$ is a numerical constant and $\tau_{\mathrm{dis}} \equiv R_{\mathrm{eo}}^{2} / D$ stands for the single-chain relaxation time. 
If the single-chain motion were described by the Rouse model, the dynamics of diblock copolymer molecules parallel and perpendicular to a planar interface of the lamellar morphology would perfectly decouple [164]. The dynamics parallel to the interfaces would be identical to the behavior in the disordered phase. In a dense melt of long polymers the molecular motion, however, is not Rouse-like but chains in a dense melt reptate along their contour. The tube-like constraint of lateral motion due to the presence of neighboring macromolecules slows down their motion in a disordered system, but it has even more pronounced effects for diblock copolymers at an interface [163]. Typically, a diblock copolymer in a lamellar phase extends its two blocks in the corresponding domains and the junction point is localized at the $A B$ interface. The slithering-snake like motion of a chain in the disordered bulk will displace the junction point away from the interface and entails a large enthalpic free-energy cost. Alternatively, one chain end has to retract to the $A B$ interface to disentangle from its original tube. This block retraction is hampered by a large entropic free-energy cost. Therefore, the motion in the lamellar phase is dramatically slowed down compared to the disordered phase and the molecular motions parallel and perpendicular to the interfaces are strongly coupled.

There are several strategies to incorporate entanglement effects into soft coarse-grained models. One approach consist in explicitly checking for bond crossing during the motion of the coarse-grained segments. This technique has been championed by Briels and co-workers $[140,141]$ and an efficient implementation has been devised by Ramanathan and Morse [165]. For very dense systems, however, this technique becomes computationally expensive, and one cannot vary the entanglement density independently from the other static properties.

An alternative approach that has been devised for polymer networks and melts by Panyukov and Rubinstein [166, 167], Schieber [142-145], and Likhtman [146, 147], respectively. It consists in mimicking the effect of entanglements by "tethering" the molecular contour to its tube by slip-links. The slip-links are harmonic springs-one end of the $j^{\text {th }}$ slip-link is anchored at a fixed position, $\mathbf{a}_{j}$ in space while the other end is located on a polymer segment $\mathbf{r}_{i_{j}, s_{j}}$. The polymer can reptate through the slip-link and the end of the slip-link hops from one segment to a neighboring one along the contour of a polymer. The partition function of the system of coarse-grained segments and slip-links takes the form

$$
Z=\int \mathcal{D}\left[\left\{\mathbf{r}_{i, s}\right\}\right] \mathcal{D}\left[\left\{\mathbf{a}_{j}, i_{j}, s_{j}\right\}\right] e^{-\frac{\mathcal{H}_{\mathrm{b}}+\mathcal{H}_{\mathrm{nb}}}{k_{B} T}} \exp \left(-\frac{3}{2 a^{2}} \sum_{j=1}^{n_{\mathrm{SL}}}\left[\mathbf{a}_{j}-\mathbf{r}_{i_{j}, s_{j}}\right]^{2}\right)
$$

where $n_{\mathrm{SL}}$ denotes the number of slip-links and $a^{2}$ is the mean-square extension of the sliplink tether, which is proportional to the tube diameter. In our simulation the average distance between slip-links is 4 coarse-grained segments, i.e., $n_{\mathrm{SL}}=n N / 4$ [149]. If we decreased the number of slip-links further or decreased $a$, the tube would become "tight" and the Rouselike motion inside the tube could not be observed. In this limit, the dynamics approaches the slithering-snake dynamics. In the opposite limit, the chains would be only weakly entangled and the dynamics would resemble the local unconstraint dynamics in the absence of sliplinks.

Since the integral over the degrees of freedom of the slip-links only yields a factor that does not depend on the particle coordinates, $\left\{\mathbf{r}_{i, s}\right\}$, the equilibrium properties are not affected by the presence of the slip-links. In order to sample the configuration space of the degrees of freedom associated with the slip-links the following two types of Monte Carlo moves can be introduced: (i) Slip-links may hop along the chain contour, i.e., $s_{j} \rightarrow s_{j} \pm 1$. This move mimics the slithering of the molecular contour through the slip links. Since this Monte Carlo move only alters $s_{j}$ it does not sample the whole configuration space of slip-links and 

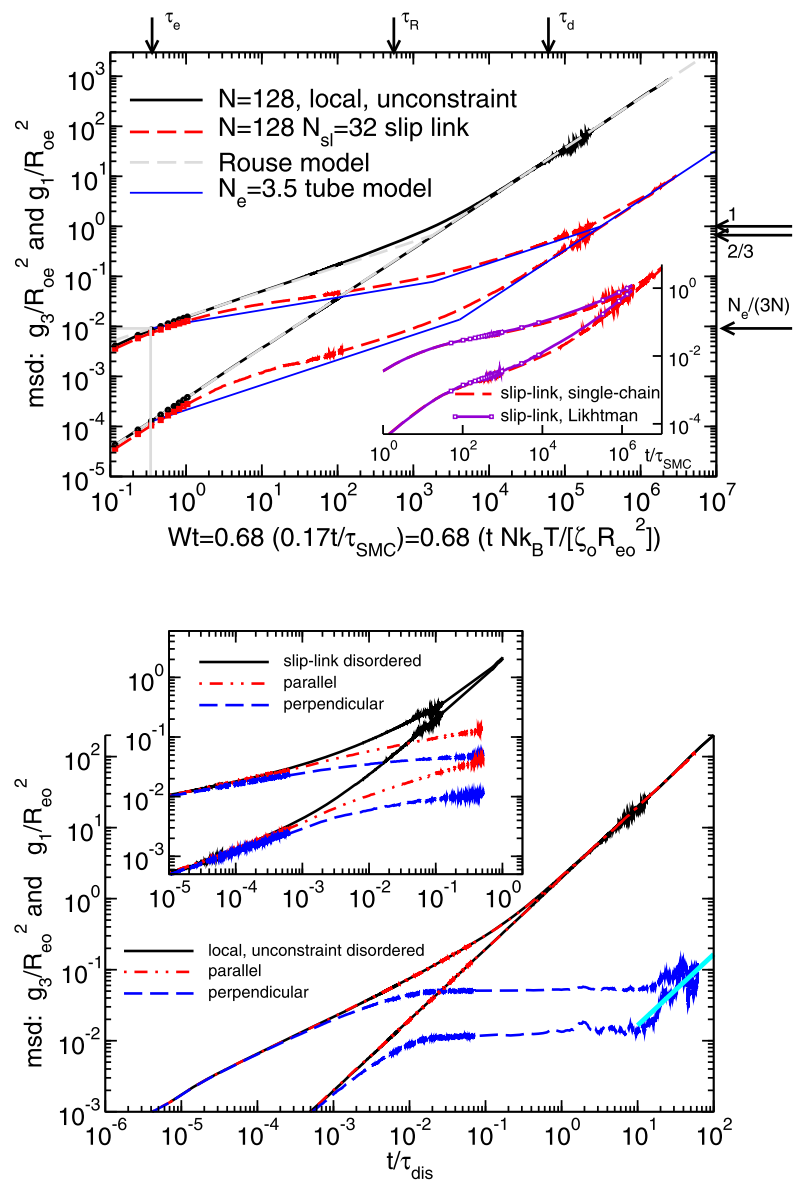

Fig. 4 (Color online) (a) Comparison of the mean-square displacements in the simulations to the predictions of Rouse-model (grey dashed lines) and tube model (blue solid lines). The mobility, $W$, and the entanglement length, $N_{\mathrm{e}}$ are identified by matching the center-of-mass diffusion coefficient to the predictions of the Rouse and the tube model, respectively. The symbols mark the simulation data at $1,2, \ldots, 9$ Monte Carlo steps (MCS). Arrows on top mark the entanglement time, $W \tau_{\mathrm{e}}=\pi N_{\mathrm{e}}^{2} / 108$, the Rouse time, $W \tau_{\mathrm{R}}=N^{2} /\left(3 \pi^{2}\right)$, and the disengagement time, $W \tau_{\mathrm{d}}=N^{3} /\left(\pi^{2} N_{\mathrm{e}}\right)$ using the value $N_{\mathrm{e}}=3.5$ extracted from the self-diffusion coefficients. The inset compares the mean-square displacements of our single-chain implementation of constraint release and the model by Likhtman [146]. (b) Mean-square displacements of segments, $g_{1}(t)$, and mean-square displacements of the center of mass, $g_{3}(t)$, as a function of time in the lamellar phase $\left(\chi_{\mathrm{o}} N=80\right.$ ) for local, unconstraint dynamics (main panel) and slip-link dynamics (inset). Parallel and perpendicular displacements are shown as well as the results in the disordered phase. "Time" is measured in units of the diffusion time, $\tau_{\mathrm{dis}}$, in the disordered morphology. The thick solid line in the main panel indicates the diffusive motion of molecules hopping between lamellae. Reprinted with permission from Ref. [149], Copyright 2008, American Institute of Physics

it does not allow the macromolecules to diffuse over long distances. (ii) In order to sample the full configurational integral, slip-links have to be created and destroyed. Since a physical entanglement between two chains corresponds to two slip-links, slip-links are grouped in pairs and their creation/deletion is a pairwise operation. If a slip-link attempts to "hop-off" a chain molecule at one end, i.e., $s_{j}=N \rightarrow s_{j}=N+1$ or $s_{j}=1 \rightarrow s_{j}=0$, this slip-link 
and the its partner in the pair are deleted, and a new pair of slip-links is created according to the Boltzmann weight of (91). This Monte Carlo move mimics the physical processes of tube renewal at the chain ends and the concomitant constraint release by the deletion of the pair partner.

In Fig. 4(a) and (b) we compare the dynamics with and without slip-links in the disordered phase and a lamellar phase of a symmetric diblock copolymer [149]. We use Smart Monte Carlo (SMC) moves [168] to update the positions of the coarse-grained segments. To this end, we use the force, $\mathbf{K}$, that acts on a segment to propose a trial displacement like in an Euler scheme for Brownian dynamics.

$$
\Delta \mathbf{r}=\mathbf{K} \frac{\Delta t}{\zeta_{\mathrm{o}}}+\Delta \mathbf{R}
$$

where $\zeta_{\mathrm{o}}$ denotes the segmental friction coefficient and $\Delta \mathbf{R}$ is Gaussian distributed with zero mean and variance $\left\langle\Delta \mathbf{R}^{2}\right\rangle=6 k_{B} T \frac{\Delta t}{\zeta_{0}}$. The moves are then accepted or rejected according to a Monte Carlo lottery, which guarantees that the Monte Carlo algorithm reproduces the exact thermodynamic equilibrium distribution independent from the "time step", $\Delta t$, of the Euler scheme. If the time step, $\Delta t$, is chosen very small, almost every Monte Carlo move will be accepted and the realization of the stochastic process will closely resemble Brownian dynamics. Since every SMC-step leads only to a tiny displacement, the mean-square displacement of a segment per CPU-time is very small. If, on the contrary, the "time step" is chosen excessively large, the algorithm will propose large segmental displacements but most of them will be rejected. In this case, the mean-square displacement will also be small per unit of CPU-time because of the low acceptance rate of the SMC moves. For our soft coarse-grained model, the optimal value is reached for $\tau_{\mathrm{SMC}}=\Delta t_{\mathrm{opt}}=0.17 \zeta_{\mathrm{o}} R_{\mathrm{eo}}^{2} /\left(N k_{B} T\right)$. Under these conditions, the mean-square displacement per CPU-time is maximal, the acceptance rate of the SMC moves is about $60 \%-70 \%$, and the segmental motion without slip-links resembles the prediction of the Rouse model (cf. Fig. 4(a)) except for the first 10 SMC steps and subtle correlation-hole effects described by $(90)[77,78]$.

Panel (a) of Fig. 4 depicts the dynamics in the disordered phase. Without slip-links, the mean-square displacement of segments, $g_{1}(t)$, and the mean-square displacement of the center-of-mass, $g_{3}(t)$, approximately follow the predictions of the Rouse-model. For the choice of parameters, correlation hole effects, which result in the anomalous diffusion described by (90), are rather small: (i) The effect, $g_{3}(t) \sim t^{3 / 4}$, is most important at early times where our simulations exhibit deviations due to the discreteness of the SMC moves. (ii) The amplitude of the corrections is small compared to other simulation studies because the invariant degree of polymerization, $\overline{\mathcal{N}}$ is large. Including slip-links, we significantly slow down the dynamics, and we can observe the different power-laws for $g_{1}$ and $g_{3}$, which have been predicted by the tube model $[87,169]$. With a moderate computational effort, we can follow the time evolution of the system for $10^{7} \mathrm{SMC}$ steps. In order to relate the SMC-steps to an experimental time, we can map the longest relaxation time of the single-chain motion, $\tau_{\text {dis }}=R_{\mathrm{eo}}^{2} / D=141000 \tau_{\mathrm{SMC}}$ for $N=128$ without slip-links, where $D$ denotes the selfdiffusion coefficient. Using $R_{\mathrm{eo}}=50 \mathrm{~nm}$ and a typical experimental value of $D=10 \mathrm{~nm}^{2} / \mathrm{s}$, one SMC-step corresponds to $1.8 \mathrm{~ms}$, and we can reach time scales on the order of hours simulating for up to $10^{7} \tau_{\mathrm{SMC}}$. Therefore, the typical experimental time scales pertinent to the self-assembly and ordering kinetics of block copolymers can be addressed by our soft coarse-grained model.

Panel (b) of Fig. 4 shows the mean-square displacements in the lamellar phase at $\chi_{\mathrm{o}} N=20$. Without slip-links (cf. main panel), the single-chain motions parallel and perpendicular to the lamellae decouple. The motion parallel to the lamellae is unaltered by the 
presence of the interfaces. At very early times, also the motion in the perpendicular direction follows the bulk behavior, but the molecules quickly realize that they are confined into the lamellae. This confinement gives rise to a plateau in the mean-square displacements, $g_{1, \perp}$ and $g_{3, \perp}$, at intermediate times. On long time scales, the molecules can hop from one lamella to a neighboring one, and diffusive behavior perpendicular to the lamellae is restored. Since this hopping process involves the tunneling of an $A$ block through a $B$-rich domain or vice versa, it is associated with a large free-energy barrier, and we can only observe the onset of diffusion in the perpendicular direction.

When we use slip-links (cf. inset of Fig. 4(b)), the motion is dramatically slowed-down both in parallel and in perpendicular direction, and the motion in the two directions is coupled. Thus the slip-link model is an efficient strategy to mimic the effect of topological constraints on the single-chain motion [149]. It also captures the effect of entanglements on the stress relaxation. In the soft coarse-grained model the largest contribution to the stress arises from the strong bonded forces; the contribution of the non-bonded interaction is rather small. Therefore the soft coarse-grained model captures one important characteristics of polymer materials with a large invariant degree of polymerization. From the autocorrelation function of the intramolecular stress, which can be computed via the virial of the bonded forces, we can obtain viscoelastic properties of the polymer liquid. The simulation data of the stress autocorrelation for the model without slip-links nicely agree with the prediction of the Rouse-model. In the case of slip-links, one can clearly observe a plateau in an intermediate time regime, which characterizes the elastic properties of the entangled macromolecular fluid in this time window [146, 149]. In the simulations, we used $N_{\mathrm{sl}}=n_{\mathrm{SL}} / n=32$ slip-links per chain, and we observe a plateau value of the stress $G^{*}=0.14(5) G_{\mathrm{o}}$ with $G_{\mathrm{o}} / N=k_{B} T \sqrt{\overline{\mathcal{N}}} / R_{\mathrm{eo}}^{3}$. For long flexible polymers, there exists a correlation between the experimentally observed plateau modulus and the packing length [154, $155,157], G^{*} / G_{\mathrm{o}}=0.00226 \overline{\mathcal{N}} / N$, which leads to a purely dynamic relation between the invariant degree of polymerization and the chain discretization or number of slip-links per chain. For our model we obtain $\overline{\mathcal{N}} \approx 62 N$ for $N_{\mathrm{SL}} / N=1 / 4$.

In Fig. 5 we illustrate the interplay between the single-chain dynamics and the ordering kinetics by investigating the directed assembly of a symmetric block copolymer on a patterned substrate. These simulations are motivated by experiments that have studied the ordering of nearly symmetric PS-PMMA diblock copolymers on a stripe pattern with a periodicity of $L_{\mathrm{o}}=48 \mathrm{~nm}$ [92]. Defect-free order and registration with the substrate pattern is established in 6 hours [92]. At 3 hours, however, one observes a pattern of spots on the top surface of the film that align with the stripe pattern of the substrate. Earlier simulations have revealed that the morphology at intermediate times signals the breaking up of the initial ordering, during which the stripe morphology is rapidly established at the chemically patterned bottom surface and the negative pattern is created at the non-preferential top surface (checkerboard morphology), i.e., the stripe pattern at the top surface is displaced by half a period with respect to the bottom morphology. This intermediate structure at the top of the film is eliminated not by lateral motion of defects but, rather, by "pushing out" the misaligned domains at the top surface. The breaking-up of the misaligned stripes at the top surface gives rise to the lines of spots in the experiment [92].

The different columns of Fig. 5 illustrate the morphologies at different stages in the course of the ordering process. The left column corresponds to local unconstraint dynamics that is described by the Rouse model. The middle column depicts morphologies obtained with the slip-link model, and the right column shows the result of simulations that use slithering-snake moves to update the molecular conformations. The latter scheme mimics a very tightly entangled system. Each row shows the morphology at the same time when 

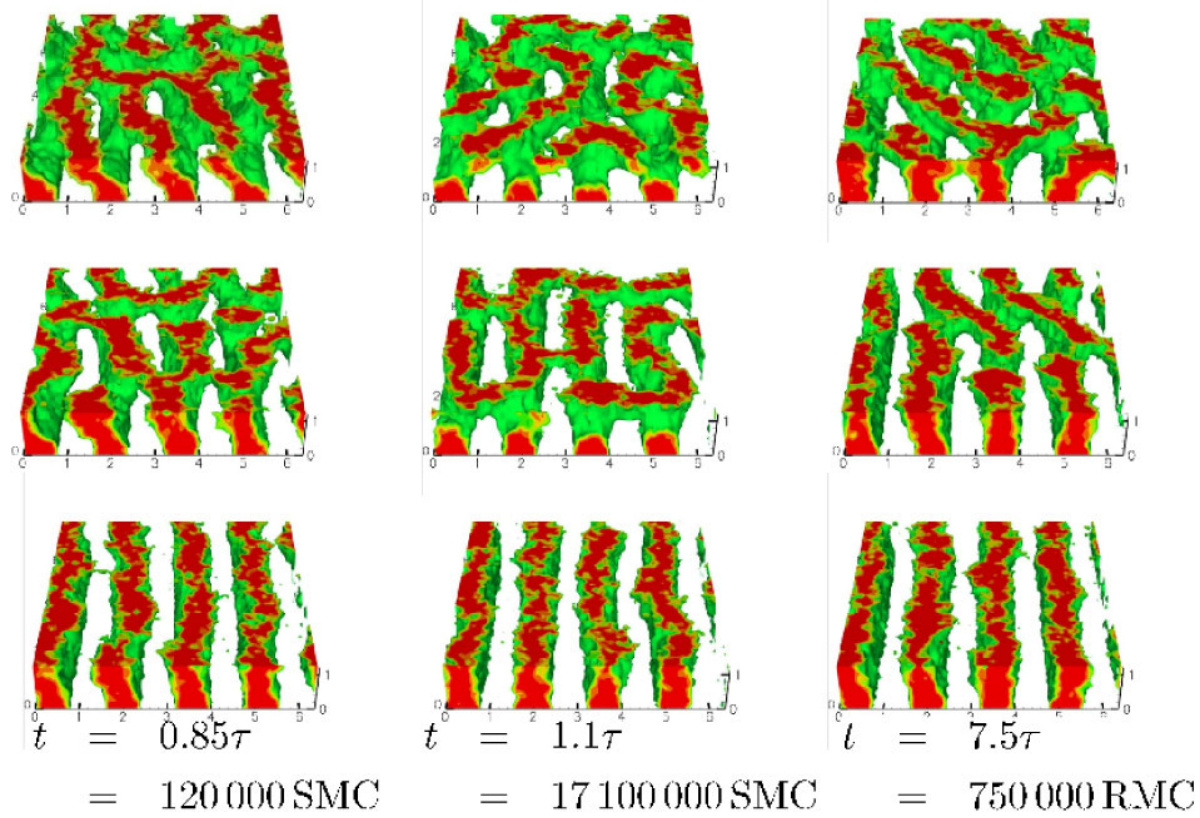

Fig. 5 Structure formation of a symmetric diblock copolymer $\left(\chi_{\mathrm{o}} N=80\right)$ on a stripe-patterned surface. The three columns correspond to local SMC-moves (a, left), slip-link simulations (b, middle), and the slithering-snake algorithm. $N=128$ and $\overline{\mathcal{N}}=128^{2}$. The three images depict morphologies at $t_{\text {ord }} / 3,2 t_{\text {ord }} / 3$ and the time, $t_{\mathrm{ord}}$, at which defect-free ordering is observed. The ordering time, $t$, is given in units of the bulk relaxation time, $\tau_{\text {dis }}$, in the disordered phase and the number of attempted displacements per segment

measured in units of the time, $t_{\text {ord }}$, that it takes to establish the final defect-free ordering. Statistically, the morphologies in each row are similar: The first row depicts an early stage, where the order and registration has been established at the patterned substrate but the domains on the top are unaligned. The middle row illustrates the stage, where the misaligned top structure is breaking up. Some parts of the registered lamellae propagate to the top surface but there still remain some defects at the top. The last row shows the first instance, where the topology of defect-free and registered domains has been established, and this stage defines $t_{\text {ord }}$. The independence of the sequence of morphologies suggests that the qualitative structure formation is dictated by the underlying free-energy functional, $\mathcal{F}\left[\phi_{A}, \phi_{B}\right]$, which describes the cost of a specific density distribution.

If one compares the time that is required to achieve ordering, $t_{\text {ord }}$, to the bulk relaxation time, $\tau_{\text {dis }}$, in the disordered phase, however, the different single-chain dynamics will yield greatly different ordering times. The local unconstraint dynamics, which mimics the Rouselike dynamics of short unentangled polymers, is the fastest; the ordering takes $0.85 \tau_{\text {dis }}$, i.e., the molecules have merely diffused their spatial extension. The slowing down of the singlechain motion by the combination of interfaces and slip-links increases the ordering time by $30 \%$. Mimicking a tightly entangled copolymer melt via the slithering-snake algorithm [170, 171], we observe an increase of the ordering time by a factor of almost 9 . Thus, while the sequence of morphologies remains unaltered by the single-chain dynamics in this example, the time scale of ordering is strongly affected.

These examples illustrate that slip-links are able to mimic different aspects of entanglements in polymer fluids without explicitly checking for the topological constraints. They 
introduce the effect of entanglements "by hand". By the number of slip-links one can adjust the degree of entanglement. The concept of a packing length yields a simple and purely dynamic relation between the invariant degree of polymerization and the chain lengths. Using this correlation, which has been verified by experiments [154, 155] and simulations [157], one can choose the number of slip-links or chain discretization in order to achieve an experimentally relevant degree of entanglement.

While this strategy has be successfully applied to the disordered bulk morphology, the application of the slip-link model to spatially inhomogeneous systems remains a challenge. First, the use of fixed anchor points breaks the translational invariance. Second, choosing a fixed number of slip-links per chain, one does not account for the correlation between the chain conformations and the degree of entanglements [172], e.g., there are indications that the effect of entanglement is reduced in thin films [173] or in strongly stretched polymer brushes [174]. Such correlations have been explored by analyzing static properties of inhomogeneous melts in thin films or brushes via a primitive path analysis [157, 175-180]. Conceptually, one could apply these primitive path analysis techniques to configurations of the soft coarse-grained model in order to investigate the coupling between molecular conformations and entanglements.

\section{Free-Energy Calculations}

For controlling the self-assembled structures and improve the material characteristics for applications in nanoscale device manufacturing, one would like to understand the properties of defects and the structure and thermodynamics of grain boundaries [181-186], where domains with different orientations of the periodic structure meet, or interfaces between coexisting morphologies.

Qualitatively similar questions have attracted abiding interest in crystalline solids and a wide spectrum of computer simulation techniques have successfully been devised to predict the structure and thermodynamics of hard condensed matter. In the following we illustrate some similarities and differences between computational studies of hard crystals and soft self-assembled structures and discuss to what extent simulation strategies for hard crystals can fruitfully be applied to soft self-assembled systems, which are illustrated by the prototypical example of diblock copolymer materials.

\subsection{Crystallization of Hard Matter Versus Self-assembly of Soft Matter}

The phenomenology of crystallization of hard condensed matter, e.g., the transition from a liquid to a crystal in a hard-sphere system [187], and the self-assembly of block copolymers share many common characteristics [26]. First, the order-parameter of the transition is the amplitude of the Fourier modes of the density or the composition, respectively. The dominant wave vectors characterize the periodic morphology. Moreover, the crystal adopts its ideal ordered state at zero temperatures, where the particles are localized at the ideal lattice positions. Upon increasing the temperature, one observes that the particles fluctuate around these ideal position and the behavior can be described by the model of the Einstein crystal. The ideal ordered state of the diblock copolymer material corresponds to the solution of the self-consistent field theory. In this mean-field theory, fluctuations of the composition are ignored, i.e., there are no fluctuations of the local composition inside the $A$-rich and $B$-rich domains, and there are no fluctuations of the positions of the internal $A B$ interfaces, i.e., capillary waves. 
One major difference between a hard-matter crystal and a self-assembled soft material is that in the self-assembled material the constituent particles are not localized around ideal crystal positions but that they can diffuse inside the domains of the structured liquid (cf. Fig. 4(b)). Thus the ideal ordered state of the self-assembled material is not a suitable reference state, whose free energy can be easily computed.

The ideal disordered state of a hard-matter crystal is the ideal gas, where the particles do not interact. The analog of this ideal disordered state of a block copolymer melt is the corresponding homopolymer melt, where the two blocks are indistinguishable and, hence, there is no driving force for structure formation. Like in the case of the ideal ordered state, the free energy of an ideal disordered crystal, i.e. an ideal gas, is readily available, but the calculation of the free energy of the ideal disordered block copolymer melt, i.e., a homopolymer melt, is a formidable task.

\subsection{The Fluid Analog of the Einstein Model}

Wilding and Bruce [188] devised a strategy to overcome this difficulty. The idea consists in "tethering" particles of the fluid to an arbitrary but fixed reference configuration of the liquid, $\left\{\mathbf{R}_{i, s}\right\}$, where the index runs over all $n N$ particles. In the liquid self-assembled material the particles diffuse far from the reference position, with which they are initially associated and the concomitant original "tether" would be pulled long. This effect can be avoided by an "association swap", where the particle initially tethered to reference position $i, s$ is subsequently associated with reference position $j, t$ and vice versa. This association-swap Monte Carlo move permits the particles to sample all positions and the tethers to respond accordingly. Wilding and Bruce employed this technique to study the freezing of hard spheres [188]. Schilling and Schmid [189] used this idea to compute the absolute free energy of the liquid. To this end, they defined the system of non-interacting particles, which are associated with an arbitrary but fixed configuration of the liquid as reference system. This reference system is the analog of the Einstein model of hard crystals except that (i) the ideal positions are now given by a representative fixed liquid configuration (instead of the ideal crystal sites) and that (ii) the association of a particle with a reference position can be altered by association swaps. The Hamiltonian of this reference system is

$$
\mathcal{H}_{\mathrm{EC}}\left(\left\{\mathbf{r}_{i, s}\right\}\right)=\varepsilon k_{B} T \sum_{i, s} \Phi_{\mathrm{o}}\left(\mathbf{r}_{i, s}-\mathbf{R}_{i, s}\right)
$$

where $\varepsilon k_{B} T \Phi_{\mathrm{o}}(\mathbf{r})$ denotes the tether potential. The free energy of this system of noninteracting particles is readily obtained

$$
F_{\mathrm{EC}}=k_{B} \operatorname{Tn} N\left[\ln \left(\frac{n N \Lambda_{T}^{3}}{V}\right)-1-\ln \left(\frac{1}{V} \int \mathrm{d}^{3} \mathbf{r} e^{-\lambda \Phi_{\mathrm{o}}(\mathbf{r})}\right)\right]
$$

One can relate this reference system to the original system via thermodynamic integration using the intermediate Hamiltonian

$$
\mathcal{H}_{\lambda}\left(\left\{\mathbf{r}_{i, s}\right\}\right)=\lambda \mathcal{H}\left(\left\{\mathbf{r}_{i, s}\right\}\right)+(1-\lambda) \mathcal{H}_{\mathrm{EC}}\left(\left\{\mathbf{r}_{i, s}\right\}\right)
$$

$\lambda=0$ corresponds to the reference system with known free energy, and $\lambda=1$ corresponds to the original system. Thus the free energy of the original system is given by

$$
F=F_{\mathrm{EC}}+\int_{0}^{1} \mathrm{~d} \lambda\left\langle\mathcal{H}\left(\left\{\mathbf{r}_{i, s}\right\}\right)-\mathcal{H}_{\mathrm{EC}}\left(\left\{\mathbf{r}_{i, s}\right\}\right)\right\rangle_{\lambda}
$$

where the average $\langle\cdots\rangle_{\lambda}$ is performed using the intermediate Hamiltonian, $\mathcal{H}_{\lambda}$. 


\subsection{The Field-Theoretic Einstein Crystal}

An alternative way to estimate the free energy of a self-assembled system consists in first converting the particle-based description into a field-theoretic continuum one, where not the coordinates, $\left\{\mathbf{r}_{i, s}\right\}$, of the coarse-grained segments but the composition fields, $\phi_{A}(\mathbf{r})$ and $\phi_{B}(\mathbf{r})$, are considered to be the fundamental degrees of freedom (see (7) and (8)). For simple model systems-like the standard model of an incompressible melt of Gaussian diblock copolymers with zero-ranged pairwise interactions [122]—one can analytically achieve such a reformulation of the particle-based partition function into a field-theoretic description by a Hubbard-Stratanovich transformation [96, 190, 191].

Since this mapping guarantees that the partition function of the particle-based and the field-theoretic description are identical, one can compute the free energy of the selfassembled morphology in the field-theoretic framework. Lennon, Katsov and Fredrickson [34] realized that the local density, $\phi_{A}(\mathbf{r})$, in a field-theoretic description fluctuates around the solution of the mean-field theory, $\phi_{A}^{*}(\mathbf{r})$, like a particle in a hard-matter crystal fluctuates around its ideal lattice position. In order to exploit this analogy, they discretize space into cells of finite volume, $\Delta L^{3}$ (cf. Sect. 2.6). The field-theoretic analog of the Einstein crystal is the reference system described by

$$
\frac{\mathcal{F}_{\mathrm{EC}}\left[\phi_{A}, \phi_{B}\right]}{k_{B} T \sqrt{\overline{\mathcal{N}}}}=\frac{\varepsilon N}{2} \frac{\Delta L^{3}}{R_{\mathrm{eo}}^{3}} \sum_{\mathbf{c}}\left(\left[\phi_{A}(\mathbf{c})-\phi_{A}^{*}(\mathbf{c})\right]^{2}+\left[\phi_{B}(\mathbf{c})-\phi_{B}^{*}(\mathbf{c})\right]^{2}\right)
$$

The strengths of the fluctuations around the mean-field solution is controlled by the parameter, $\varepsilon N$, which can be chosen such that the density fluctuations in the reference system match those of the original system. ${ }^{2}$ The free energy of the reference system is given by ${ }^{3}$

$$
\begin{aligned}
F_{\mathrm{EC}} & \equiv-k_{B} T \ln \int \mathcal{D}\left[\phi_{A}, \phi_{B}\right] e^{-\frac{\mathcal{F}_{\mathrm{EC}}\left[\phi_{A}, \phi_{B}\right]}{k_{B} T}} \\
& =-2 k_{B} T \frac{V}{\Delta L^{3}} \ln \int \mathrm{d} \varphi \exp \left(-\frac{\varepsilon N}{2} \frac{\Delta L^{3}}{R_{\mathrm{eo}}^{3}} \sqrt{\overline{\mathcal{N}}} \varphi^{2}\right) \\
& =k_{B} T \frac{V}{\Delta L^{3}} \ln \frac{\varepsilon N \Delta L^{3} \sqrt{\overline{\mathcal{N}}}}{2 \pi R_{\mathrm{eo}}^{3}}
\end{aligned}
$$

Using a parameter, $\lambda$, one relates the original system to the reference system. To this end, one studies a system with an intermediate free-energy functional

$$
\mathcal{F}_{\lambda}\left[\phi_{A}, \phi_{B}\right]=\lambda \mathcal{F}\left[\phi_{A}, \phi_{B}\right]+(1-\lambda) \mathcal{F}_{\mathrm{EC}}\left[\phi_{A}, \phi_{B}\right]
$$

and obtains the free energy via thermodynamic integration

$$
F=F_{\mathrm{EC}}+\int_{0}^{1} \mathrm{~d} \lambda\left\langle\mathcal{F}\left[\phi_{A}, \phi_{B}\right]-\mathcal{F}_{\mathrm{EC}}\left[\phi_{A}, \phi_{B}\right]\right\rangle_{\lambda}
$$

\footnotetext{
${ }^{2}$ The derivation allows to choose a different value of $\varepsilon N$ in each grid cell, $\mathbf{c}$.

${ }^{3}$ The free energy becomes singular in the limit $\Delta L \rightarrow 0$. This UV-divergence prevents the calculation of absolute free energies [192], but free-energy differences between different morphologies with the same spatial resolution, $\Delta L$, are well defined.
} 
Lennon, Katsov and Fredrickson [34] used this technique to explore fluctuation effects on the order-disorder transition of diblock copolymer melts described by the EdwardsHamiltonian of Gaussian chains with zero-ranged interactions. For this specific model, a free-energy functional $\mathcal{G}\left[w_{A}, w_{B}\right]$ in terms of the fields, $w_{A}(\mathbf{r})$ and $w_{B}(\mathbf{r})$, that are conjugated to the densities, $\phi_{A}(\mathbf{r})$ and $\phi_{B}(\mathbf{r})$, has been analytically derived by a HubbardStratanovich transformation $[193,194]$. Unfortunately, $\mathcal{G}$ is complex-valued and the concomitant statistical weight, $\exp \left(-\mathcal{G} / k_{B} T\right)$, is not positive definite. Thus special simulation techniques - complex Langevin simulations - have to be employed to sample the field configurations and cope with the sign-problem due to the non-positive statistical weights [195].

In Sect. 2.4 we have outlined a computational strategy of deriving a free-energy functional, $\mathcal{F}\left[\phi_{A}, \phi_{B}\right]$, from an arbitrary particle-based description by computer simulation (e.g., the interactions may also include hard excluded volume interactions like the Lennard-Jones potential). The technique of the field-theoretic Einstein crystal can be applied to the soderived real free-energy functional in order to map out the phase diagram or the free energy of defects.

\subsection{Reversible Transformation of Self-assembled Morphologies}

The free-energy differences that dictate the relative stability of two competing morphologies typically are much smaller than the absolute free energies of the morphologies. In order to estimate phase boundaries one needs an accuracy of $10^{-2} k_{B} T$ per macromolecule. The main contribution to this free energy stems from the liquid structure of the coarse-grained polymer melt, i.e., even in a soft coarse-grained model it is on the order of $k_{B} T$ per segment. Hence, given that $N \sim \mathcal{O}\left(10^{2}\right)$, we need to know the absolute free energy with a relative accuracy of the order $10^{-4}$. This is a formidable challenge. In order to omit the large contribution of the fluid structure, it is advantageous that we directly compare the free energy of two competing morphologies rather than relating the free energy of a self-assembled morphology to that of a reference state and computing the free-energy difference by the subtraction of two large numbers.

In the parameter space of physical variables, e.g., $\chi_{\mathrm{o}} N$ or temperature, the transition between different morphologies is of first order. Even the order-disorder transition in a symmetric diblock copolymer, which the mean-field theory predicts to be of second-order [100], is a fluctuation-induced transition of first order [64]. Thus, in the parameter space of physical variables, one cannot construct a reversible path that relates different morphologies of a self-assembled system. Likewise there is no simple physical order-parameter (like the global composition in a binary mixture undergoing macroscopic phase separation [196]) that parameterizes a reversible path of phase transformation from one state to another coexisting state in a finite system. ${ }^{4}$

For hard-matter crystals, Sheu, Lou, and Lovett devised a method how a solid can be turned into a gas without passing through a first-order phase transformation [198]. This reversible thermodynamic path between the solid and a liquid state has been rediscovered and successfully applied to various hard-matter systems by Grochola and coworkers [199]. This strategy can also be transferred to soft self-assembled systems [26, 28, 30].

The reversible transformation of one self-assembled morphology into another one is based on the application of a specifically designed, spatially inhomogeneous, external field. To illustrate the method (see Fig. 6), let us consider the free-energy difference between an

\footnotetext{
${ }^{4}$ Progress towards this goal has recently been achieved in the framework of self-consistent field theory [197].
} 


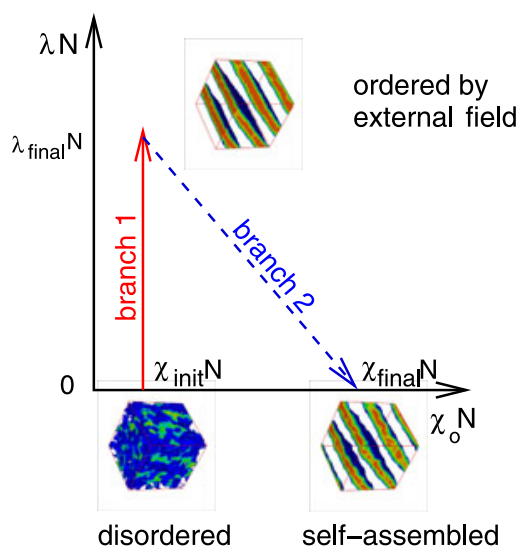

Fig. 6 Sketch of the reversible path that connects the homogeneous disordered state, the externally ordered and the self-assembled state. Configurational snapshots of a symmetric $A B$ diblock melt illustrate the different states. In the snapshots three-dimensional contour plots of the composition are shown. The $B$-rich component is removed for clarity, and the interface between the different components is colored blue. $\chi_{\mathrm{o}} N=0$, $\chi_{*} N=20$, and the maximal strength of the ordering field is $\lambda_{*} N=10$. The SCMF simulations correspond to $\overline{\mathcal{N}}=14884$ and use a chain discretization, $N=32$. The linear extension of the simulation cell is $L=4.77 R_{\mathrm{eo}}$ and the spacing of the lamellar structure is $L_{\mathrm{O}}=L /(2 \sqrt{2})=1.686 R_{\mathrm{eo}}$. Reprinted with permission from Ref. [26], Copyright 2008, American Institute of Physics

ideal disordered phase, i.e. a homopolymer melt with $\chi_{\mathrm{o}} N=0$, and a self-assembled morphology at $\chi_{\mathrm{o}} N=\chi_{*} N$. As we change the incompatibility, $\chi_{\mathrm{o}} N$, between $A$ and $B$ segments there is a first-order transition from the disordered morphology to a spatially modulated morphology, e.g., a lamellar structure for a symmetric diblock copolymer. Thus, increasing $\chi_{0} N$ from 0 to $\chi_{*} N$, we do not reversibly transform a disordered into a spatially modulated morphology. In the extended parameter space of incompatibility, $\chi_{\mathrm{o}} N$, and strength, $\lambda N$, of an external field, however, such a reversible path can be constructed as follows:

First, we use the external ordering field to structure the homopolymer melt with $\chi_{\mathrm{o}} N=0$ into the spatially modulated morphology. To this end, we add to the bonded and non-bonded Hamiltonian of the soft coarse-grained model an external ordering field

$$
\begin{aligned}
\mathcal{H}_{\text {ext }}\left(\left\{\mathbf{r}_{i, s}\right\}\right) & =\mathcal{F}_{\text {ext }}\left[\hat{\phi}_{A}\left(\mathbf{r} \mid\left\{\mathbf{r}_{i, s}\right\}\right), \hat{\phi}_{B}\left(\mathbf{r} \mid\left\{\mathbf{r}_{i, s}\right\}\right)\right] \quad \text { with } \\
\frac{\mathcal{F}_{\text {ext }}\left[\phi_{A}, \phi_{B}\right]}{k_{B} T \sqrt{\overline{\mathcal{N}}}} & =-\lambda N \int \frac{\mathrm{d}^{3} \mathbf{r}}{R_{\text {eo }}^{3}} h(\mathbf{r})\left[\phi_{A}(\mathbf{r})-\phi_{B}(\mathbf{r})\right]
\end{aligned}
$$

$\lambda N$ quantifies the strength of the external field, and $h(\mathbf{r})$ characterizes its spatial modulation. Positive values, $h>0$, correspond to regions that attract $A$ segments while negative values characterize spatial domains, in which $B$ segments are enriched. The external field should be chosen such that the structure at $\chi_{0} N=0$ and $\lambda N=\lambda_{*} N$ closely resembles the structure of the spatially modulated phase of interest at $\chi_{\mathrm{o}} N=\chi_{*} N$ in the absence of the external ordering field, $\lambda N=0$.

The system at $\chi_{\mathrm{o}} N=0$ and $\lambda N=\lambda_{*} N$, i.e., the end of the first branch of the transformation path, mimics the system considered in the self-consistent field theory, where the $A B$ interaction of a macromolecule with its neighbors is approximated by a static, external field. Thus we can use the mean-field theory to obtain a first approximation for the external field 
that generates the spatially modulated structure in the system without $A B$ repulsion

$$
\begin{aligned}
2 \lambda_{*} N h(\mathbf{r}) & \approx-\frac{R_{\mathrm{eo}}^{3}}{k_{B} T \sqrt{\overline{\mathcal{N}}}}\left(\frac{\delta \mathcal{F}_{\mathrm{nb}}}{\delta \phi_{A}(\mathbf{r})}-\frac{\delta \mathcal{F}_{\mathrm{nb}}}{\delta \phi_{B}(\mathbf{r})}\right) \\
& =\chi_{*} N\left\langle\hat{\phi}_{A}\left(\mathbf{r} \mid\left\{\mathbf{r}_{i, s}\right\}\right)-\hat{\phi}_{B}\left(\mathbf{r} \mid\left\{\mathbf{r}_{i, s}\right\}\right)\right\rangle_{\chi_{0} N=\chi_{*} N, \lambda N=0}
\end{aligned}
$$

where we have used the specific form of the non-bonded interactions, (63). For polymer systems with a large value of $\overline{\mathcal{N}}$, the mean-field approximations is rather accurate, and this estimate can readily be used in the simulations. For lipid systems, however, fluctuations of the density and liquid-like packing effects are more important and give rise to deviations from the mean-field predictions [30]. In this case, the mean-field estimate provides an initial guess, but to determine the external ordering field that accurately reproduces the self-assembled morphology one iteratively adjusts $\lambda_{*} N h(\mathbf{r})$.

Since the structuring of the polymer liquid occurs in response to an external field, $\lambda N=$ $0 \rightarrow \lambda_{*} N$, the structure formation is not a collective phenomena-each molecule arranges into the modulated structure due to the external field rather than in response to the nonbonded interaction with its neighbors. ${ }^{5}$ Thus the assembly by the external field is reversible, and one can obtain the free-energy difference along this first branch of the transformation path by thermodynamic integration.

$$
\frac{\Delta F_{1}}{k_{B} T \sqrt{\overline{\mathcal{N}}}}=-\int_{0}^{\lambda_{*} N} \mathrm{~d} \lambda N \int \frac{\mathrm{d}^{3} \mathbf{r}}{R_{\mathrm{eo}}^{3}} h(\mathbf{r})\left\langle\hat{\phi}_{A}\left(\mathbf{r} \mid\left\{\mathbf{r}_{i, s}\right\}\right)-\hat{\phi}_{B}\left(\mathbf{r} \mid\left\{\mathbf{r}_{i, s}\right\}\right)\right\rangle_{\lambda N}
$$

Along the second branch, the external ordering field is switched off and the repulsion between $A$ and $B$ segments is increased in turn. Thus the effect of the external ordering field is replaced by the driving force for self-assembly. Optimally, this is done in such a way that the structure of the system does not change. This requires that the structure at the ends of the second branch - the system assembled by the external field, $\chi_{0} N=0, \lambda N=\lambda_{*} N$, and the self-assembled system in the absence of the field, $\chi_{0} N=\chi_{*} N, \lambda N=0$-are very similar. This requirement is fulfilled by (106) for dense polymer systems. In fact, one can use mean-field theory to estimate how the optimal path, $\chi_{\mathrm{o}} N(\lambda N)$, in the plane of $A B$ repulsion and external field strength should be chosen. The condition that the structure of the system within mean-field approximation does not change, is equivalent to the molecular field of the self-consistent field theory be independent from $\lambda N$, i.e.,

$$
\chi_{\mathrm{o}} N\left\langle\hat{\phi}_{A}\left(\mathbf{r} \mid\left\{\mathbf{r}_{i, s}\right\}\right)-\hat{\phi}_{B}\left(\mathbf{r} \mid\left\{\mathbf{r}_{i, s}\right\}\right)\right\rangle_{\lambda N}+2 \lambda N h(\mathbf{r})=\mathrm{const}
$$

In conjunction with (106), the mean-field theory suggests to use $h(\mathbf{r})=\left\langle\hat{\phi}_{\mathbf{A}}\left(\mathbf{r} \mid\left\{\mathbf{r}_{\mathbf{i}, \mathbf{s}}\right\}\right)-\right.$ $\left.\hat{\phi}_{\mathbf{B}}\left(\mathbf{r} \mid\left\{\mathbf{r}_{\mathbf{i}, \mathbf{s}}\right\}\right)\right\rangle$, where the average is performed in the self-assembled system, and the simple linear path, $\chi_{0} N(\lambda N)=\chi_{*} N-2 \lambda N$ with $0 \leq \lambda N \leq \chi_{*} N / 2$ as depicted in Fig. 6 .

Since the structure does not abruptly change along the second branch, this transformation from a modulated structure assembled by the external ordering field to the self-assembled structure formed by the amphiphilicity of the constituents is reversible. The concomitant

\footnotetext{
${ }^{5}$ In contrast to the fluid analog of the Einstein crystal in Sect. 3.2, the external ordering field localizes $A$ segments in spatial domains, which are defined by $h(\mathbf{r})$, rather than tethering them to specific reference positions in space.
} 
free-energy change can also be obtained by thermodynamic integration. Thus, in the extended parameter space of incompatibility of the amphiphilic units and strength of the external ordering field, the two branches form a reversible path that connects the disordered morphology to a spatially modulated one.

The same strategy can also be employed to reversibly transform one spatially modulated structure into another [28, 30]. To this end, one employs two external ordering fields, $h_{1}$ and $h_{2}$, that correspond to the two self-assembled morphology. First, starting with morphology (1), one replaces the $A B$ repulsion by the external ordering field, $h_{1}$, and reversibly transforms the self-assembled morphology (1) into a very similar structure that is assembled by the external ordering field, $h_{1}$. Second, one gradually switches the external ordering field from $h_{1}$ to $h_{2}$. This transformation of the structure is reversible because the structural change occurs in response to the external ordering field and does not involve collective behavior. After completion of this reversible second branch, the system has adopted the structure (2). Third, the external ordering field that assembles structure (2) is reduced and the amphiphilic repulsion is switched on. Thereby we reversibly transform the externally assembled system into a self-assembled system.

Instead of thermodynamic integration, one can perform the free-energy calculation by a combination of expanded-ensemble and replica-exchange simulation techniques [28]. To this end, one discretizes the integration path into a set of intervals, $\left\{I_{p}\right\}$ with $p=1, \ldots, M$ and chooses sampling points $\lambda_{p, q} N$ with $q=1, \ldots, m_{p}$ in each interval. The end points of the transformation path, where the external ordering field vanishes, are included in the set of sampling points, i.e. $\lambda_{1,1} N=\lambda_{M, m_{M}}$, and neighboring intervals share a common boundary, i.e., $\lambda_{p, m_{p}} N=\lambda_{p+1,1} N$.

Each interval is associated with one replica of the system, and there are replica-exchange Monte Carlo moves that swap configurations between neighboring replicas [200-202]. Within each interval, $\lambda_{p, q} N$ is a stochastic variable, and we perform expanded-ensemble Monte Carlo moves [203] that alter the value of $\lambda_{p, q} N$. The different values of $\lambda_{p, q} N$ are weighted by a pre-weighting factor, $w_{p, q}$. These pre-weighting factors are chosen as to ensure that each sampling point within an interval is visited with roughly equal probability. The acceptance criterion of the expanded-ensemble Monte Carlo moves from sampling point $q$ to $q^{\prime}$ in interval $p$ only involves the difference, $w_{p, q}-w_{p, q^{\prime}}$. Thus we can choose $w_{1,1}=0$ and match the pre-weighting factors at the interval boundaries, $w_{p, m_{p}}=w_{p+1,1}$. The partition function of the simulation is given by [130]

$$
Z_{\mathrm{MC}} \sim \prod_{p=1}^{M} \sum_{q=1}^{m_{p}} e^{\frac{w_{p, q}}{k_{B} T}} \int \mathcal{D}\left[\left\{\mathbf{r}_{i, s}^{(p)}\right\}\right] \exp \left(-\frac{\mathcal{H}_{\mathrm{b}}+\mathcal{H}_{\mathrm{nb}}\left(\chi_{\mathrm{o}} N\left(\lambda_{p, q} N\right)\right)+\mathcal{H}_{\mathrm{ext}}\left(\lambda_{p, q} N\right)}{k_{B} T}\right)
$$

where the arguments of $\mathcal{H}_{\mathrm{nb}}$ and $\mathcal{H}_{\text {ext }}$ indicate the interaction strengths of the amphiphilic repulsion and the external ordering field, respectively. This hierarchical simulation technique is very well suited for massive parallel computer systems.

The probability, $P_{p, q}$, of finding a replica at sampling point $p, q$ is proportional to

$$
P_{p, q} \propto e^{\frac{w_{p, q}}{k_{B} T}} e^{-\frac{F\left(\chi_{0} N\left(\lambda_{p, q} N\right), \lambda_{p, q} N\right)}{k_{B} T}}
$$

where $F\left(\chi_{0} N\left(\lambda_{p, q} N\right), \lambda_{p, q} N\right)$ denotes the free energy of the system with incompatibility $\chi_{0} N\left(\lambda_{p, q} N\right)$ subjected to an external ordering field of strength $\lambda_{p, q} N$. Thus, in order to visit all sampling points with roughly equal probability, one should choose $w_{p, q} \approx$ $F\left(\chi_{\mathrm{o}} N\left(\lambda_{p, q} N\right), \lambda_{p, q} N\right)$. 
Fig. 7 Changes of the free energy along the two branches of the transformation path (thick lines). The system parameters are identical to Fig. 6. The approximate expressions based on the

Random-Phase-Approximation (RPA) and the

Strong-Segregation Limit (SSL) are also shown. Reprinted with permission from Ref. [26], Copyright 2008, American Institute of Physics

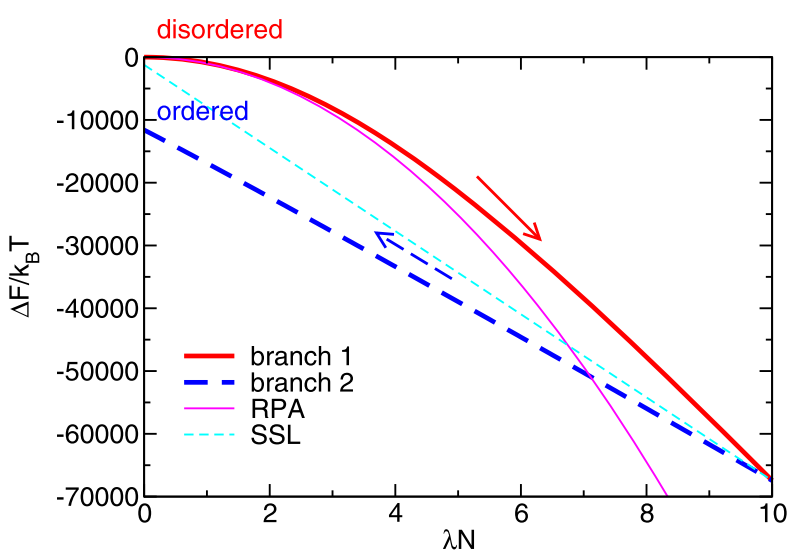

Since the free energy, $F$, is extensive, it varies over many $k_{B} T$ along the transformation path (e.g., $\Delta F \sim \mathcal{O}\left(10^{4} k_{B} T\right)$ in Fig. 7) and systematic and automated methods to iteratively obtain $w_{p, q}$ are required. The replica-exchange guarantees that there is one system in each interval. Thus one can sample the integrand of (107), or its generalization for the case that $\chi_{\mathrm{o}} N$ is also varied along the path, and use thermodynamic integration to obtain $w_{p, q}$. Alternatively, one can use Wang-Landau techniques [204] or successive umbrella sampling [205] to estimate the pre-weighting factors. Additional information about the free energy of the disordered phase structured by an external field can be obtained from the RPA (at the beginning of the first branch) or the approximation of strong segregation (at the beginning of the second branch) as illustrated in Fig. 7.

Once the pre-weighting factors have been adjusted, the free-energy difference between the end-points of the transformation path is given by:

$$
\begin{aligned}
\Delta F & \equiv F\left(\chi_{\mathrm{o}} N\left(\lambda_{M, m_{M}} N\right), \lambda_{M, m_{M}}\right)-F\left(\chi_{\mathrm{o}} N\left(\lambda_{1,1} N\right), \lambda_{1,1}\right) \\
& =w_{M, m_{M}}-w_{1,1}-k_{B} T \ln \frac{P_{M, m_{M}}}{P_{1,1}}
\end{aligned}
$$

The use of the expanded-ensemble simulations has two advantages: (i) From the observation that the system samples all values $\left\{\lambda_{p, q} N\right\}$ along the reversible path with roughly equal probability (see Fig. 8) we conclude that the accuracy of the pre-weighting factors and therefore also the accuracy of the estimate of the free-energy difference, $\Delta F$, is on the order $k_{B} T$. Since the total free-energy difference $\Delta F$ is on the order $10^{4} k_{B} T$ we achieve a relative accuracy of $10^{-4}$, which is sufficient for estimating the free energy of a bulk morphology and the free energy of grain boundaries or defects of self-assembled morphologies in thin films [28]. (ii) From the observation that the system freely diffuses along the transformation path and that the time evolution of $\lambda_{p, q} N$ does not exhibit a banded structure, we conclude that there are no barriers along the path and that the gradual replacement of the external ordering field by the amphiphilic repulsion indeed is reversible.

\subsection{Measuring the Chemical Potential in the $n P T$-Ensemble}

The previous methods have adopted simulation techniques from hard-matter systems. One strategy that exploits the softness of the segmental interactions consists in measuring the 


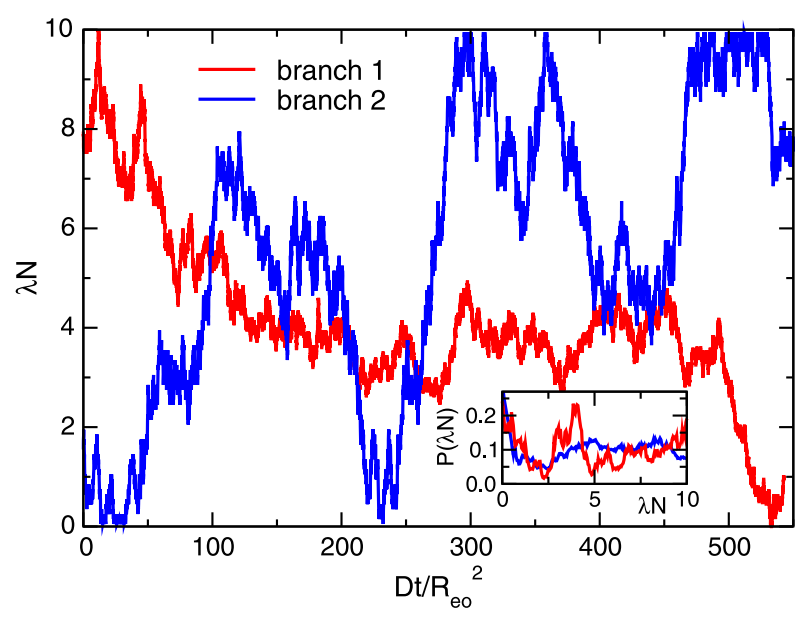

Fig. 8 Evolution of the ordering field, $\lambda N$, in the course of the expanded-ensemble simulation along both branches. The system parameters are identical to Fig. 6. Smart Monte Carlo moves are used to update the molecular conformations. The local segment motion gives rise to Rouse-like dynamics. "Time" is measured in units of the bulk relaxation time of the macromolecules in the disordered state. The inset presents the probability, $P(\lambda N)$, with which the different states, $\left(\lambda N, \chi_{\mathrm{o}}(\lambda N)\right)$, of the expanded ensemble are visited. No replica-exchange Monte Carlo moves are performed for this simulation run, and the figure presents the data for a single configuration. Reprinted with permission from Ref. [26], Copyright 2008, American Institute of Physics

chemical potential, $\mu$, of the amphiphilic molecules at constant pressure. Basic thermodynamics states that the Gibbs free energy, $G$, takes the form

$$
G(n, P, T)=\sum_{\alpha} \mu_{\alpha} n_{\alpha}
$$

where the index, $\alpha$, runs over all molecular species. The measurement of the chemical potential in a crystalline solid via Widom particle insertion [206] is impossible, but the soft interactions significantly facilitate the insertion of entire molecules, and thereby the accurate measurement of the thermodynamic potential. This strategy has been exploited by Escobedo and co-workers [32, 33, 207]. For a one-component system, the Gibbs free energy, $G$, is related to the partition function in the isothermal-isobaric ensemble

$$
e^{-\frac{G(n, P, T)}{k_{B} T}}=\frac{P}{k_{B} T} \int \mathrm{d} V e^{-\frac{P V}{k_{B} T}} \frac{V^{n}}{n ! \Lambda_{T}^{3 n N}} \prod_{i=1}^{n} \int \tilde{\mathcal{D}}_{i}\left[\left\{r_{i, s}\right\}\right] e^{-\frac{\mathcal{H}\left(\left(\mathbf{r}_{i, s}\right)\right)}{k_{B} T}}
$$

where the coordinates of the first segment of a molecule is scaled with the system size, i.e., $\tilde{\mathbf{r}}_{i, 1}=\mathbf{r}_{i, 1} / \sqrt[3]{V}$ and $\Delta \mathbf{r}_{i, s} \equiv \mathbf{r}_{i, s}-\mathbf{r}_{i, 1}$ denote the internal coordinates with respect to the first segment of a molecule. $\tilde{\mathcal{D}}_{i}\left[\left\{r_{i, s}\right\}\right] \equiv \mathrm{d}^{3} \tilde{\mathbf{r}}_{i, 1} \prod_{s=2}^{n} \mathrm{~d}^{3} \Delta \mathbf{r}_{i, s}$ is the integral over the scale position and all internal coordinates of the $i^{\text {th }}$ molecule.

The chemical potential, $\mu$, in the $n P T$-ensemble can be obtained by sampling the energy, $\Delta E$, of virtually adding one molecule, $i=n+1$, to a system comprised of $n$ molecules [206, 208]

$$
e^{-\frac{\mu}{k_{B} T}} \equiv \frac{\exp \left(-\frac{G(n+1, P, T)}{k_{B} T}\right)}{\exp \left(-\frac{G(n, P, T)}{k_{B} T}\right)}
$$




$$
=\left\langle\frac{V}{(n+1) \Lambda_{T}^{3 N}} \int \tilde{\mathcal{D}}_{n+1}\left[\left\{r_{n+1, s}\right\}\right] \exp \left(-\frac{\Delta E}{k_{B} T}\right)\right\rangle_{n, P, T}
$$

Defining the single-chain partition function of a non-interacting molecule, $n+1$, and the average of non-interacting single-chain conformations according to

$$
\begin{aligned}
\mathcal{Q}(T) & \equiv \frac{1}{\Lambda_{T}^{3(N-1)}} \int \tilde{\mathcal{D}}_{n+1}\left[\left\{r_{n+1, s}\right\}\right] \exp \left(-\frac{\mathcal{H}_{\mathrm{b}, n+1}}{k_{B} T}\right) \\
{[\cdots]_{n+1} } & \equiv \frac{1}{\mathcal{Q}(T) \Lambda_{T}^{3(N-1)}} \int \tilde{\mathcal{D}}_{n+1}\left[\left\{r_{n+1, s}\right\}\right] \exp \left(-\frac{\mathcal{H}_{\mathrm{b}, n+1}}{k_{B} T}\right) \cdots
\end{aligned}
$$

we can split the chemical potential into an ideal contribution, $\mu_{\mathrm{id}}$, and an excess part, $\mu_{\mathrm{ex}}$.

$$
\begin{aligned}
\mu & \equiv \mu_{\mathrm{id}}+\mu_{\mathrm{ex}} \quad \text { with } \\
\frac{\mu_{\mathrm{id}}(P, T)}{k_{B} T} & =-\ln \frac{\mathcal{Q}(T) k_{B} T}{P \Lambda_{T}^{3}}=\ln \frac{n \Lambda_{T}^{3}}{V_{\mathrm{id}} \mathcal{Q}(T)} \quad \text { with } \quad P V_{\mathrm{id}}=n k_{B} T \\
\frac{\mu_{\mathrm{ex}}}{k_{B} T} & =-\ln \left[\left\langle\frac{P V}{(n+1) k_{B} T} \exp \left(-\frac{\Delta E-\mathcal{H}_{\mathrm{b}, n+1}}{k_{B} T}\right)\right\rangle_{n, P, T}\right]_{n+1}
\end{aligned}
$$

The argument of the exponential, $\Delta E_{\mathrm{nb}}=\Delta E-\mathcal{H}_{\mathrm{b}, n+1}$, only comprises the change of the non-bonded interactions upon insertion of the $(n+1)^{\text {th }}$ molecule. Since these interactions are very soft, the fluctuations of $\Delta E_{\mathrm{nb}}$ are much smaller than in models with harsh excluded volume interactions and there are significantly less sampling problems. Moreover, the insertion can be combined with configurational-bias Monte Carlo techniques [209-211] or histogram analysis methods [212] in order to generate more suitable configurations of the $(n+1)^{\text {th }}$ ghost particle or to assess the quality of the insertion statistics, respectively. Since the ideal part of the chemical potential, $\mu_{\text {id }}(P, T)$, is the same in coexisting phases, one can map out the phase diagram by comparing the excess chemicals of competing structures.

Figure 9 illustrates this strategy for the fluctuation-induced first-order transition from a disordered to an ordered state of a symmetric diblock copolymer [29]. The different lines present the excess chemical potential in the isothermal-isobaric ensemble in the disordered state $\left(\right.$ low $\chi_{\mathrm{o}} N$ ) and the ordered lamellar morphology (high $\chi_{\mathrm{o}} N$ ), respectively. The figure depicts data sets for different values of the invariant degree of polymerization, $\overline{\mathcal{N}}$. The curve, $\mu_{\mathrm{ex}}\left(\chi_{\mathrm{o}} N\right)$, of the excess chemical potential that corresponds to the disordered state intersects the curve of the ordered lamellar morphology at a finite angle indicating that the transition is indeed of first order. From the intersection point one can accurately read-off the transition point. One particular advantage of the $n P T$-ensemble is that simulation cell will adjust its dimension to the stress-free self-assembled morphology if one allows the different linear dimensions to fluctuate independently. This is particularly important in this example because the equilibrium lamellar spacing strongly depends on the incompatibility, $\chi_{0} N$ in the vicinity of the phase transition.

Alternatively, one can use the relation between the grandcanonical potential, $\Omega$, and the pressure, $P$,

$$
\Omega(\mu, V, T)=-P V
$$

and measure the pressure in grandcanonical simulations [32]. Using the weighting function approach, the pressure can be determined from the virial of bonded and non-bonded forces. By the same token, the soft interactions between the coarse-grained segments allow for an efficient simulation in the $\mu V T$-ensemble. 


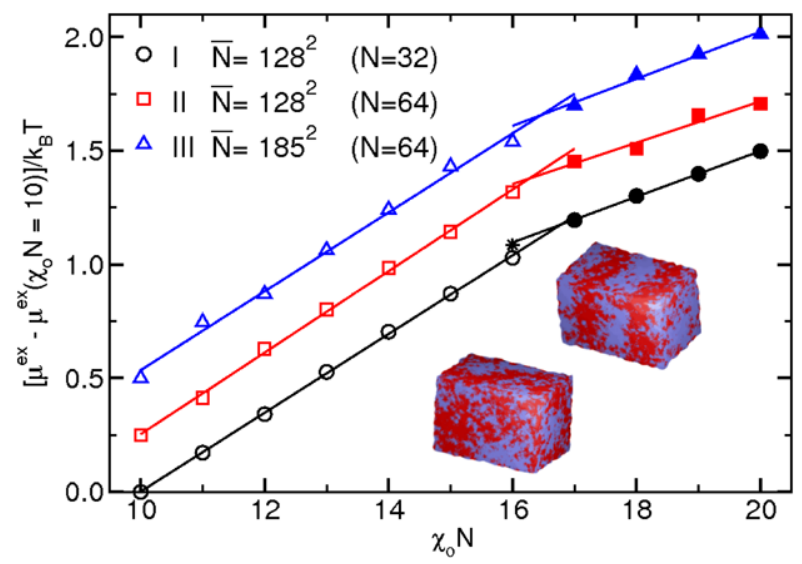

Fig. 9 Order-disorder-transition (ODT) of a symmetric diblock copolymer studied by a soft coarse-grained model. Monte Carlo simulations are performed in the $n P T$ ensemble and the pressure, $P$ kept constant at $P b^{3} / k_{B} T=18$ (with $b=R_{\text {eo }} / \sqrt{N-1}$ ). $\kappa_{\mathrm{O}}=1.5625$. The invariant degree of polymerization, $\overline{\mathcal{N}}$, and the chain discretization are indicated in the key. The figure presents the excess thermodynamic potential, $\mu_{\mathrm{ex}}$, per particle as a function of $\chi_{\mathrm{o}} N$. The curves have been shifted for clarity. Empty and filled symbols denote the disordered and lamellar phases, respectively. Lines are linear fits to the data. The crossing points identify the locations of the order-disorder transition. Errors are comparable to the symbol size. Snapshots illustrate the structure before and after the order-disorder transition (i.e., at $\chi_{\mathrm{o}} N=16$ and 17), respectively. Reprinted with permission from Ref. [29], Copyright 2009, American Institute of Physics

\section{Summary and Outlook}

In this contribution we have discussed the use of soft coarse-grained models for studying structure formation in amphiphilic systems by computer simulation. Soft potentials between coarse-grained segments that represent many monomeric repeat units of a chemically realistic model naturally arise in the course of systematically deriving a coarse-grained force field from a chemically realistic one. The main benefits of models for dense multicomponent polymer systems with soft potentials are (i) the description of experimentally relevant large values of the invariant degree of polymerization, $\overline{\mathcal{N}}$, and the concomitant description of fluctuation effects, (ii) the accessibility of large time and length scales (e.g., hours and $\mu \mathrm{m}$ for dense polymer melts), and (iii) the ease with which the Gibbs free energy or chemical potential can be measured.

One can capture the universal properties of amphiphilic self-assembly by minimal models that only incorporate the relevant interactions: molecular connectivity, repulsion between amphiphilic units, and near incompressibility of the liquid. Particularly, we have used a model where the molecular architecture is described by a bead-spring model and the nonbonded interactions are described by an excess free-energy functional, which depends on the explicit coordinates of the segments via the local microscopic densities. Advantages and disadvantages of different definitions of the local microscopic densities, based on a collocation grid or a weighting function, have been discussed with regard to computational speed, incorporation of short-ranged fluid structure and packing as well as the measurement of pressure. The model of the molecular architecture can be generalized to incorporate more complex chain architectures (i.e., polymer brushes, star or comb polymers) or include supramolecular associations [213, 214]. The molecular model can also be augmented e.g., by a bond-stiffness potential for a better description of the local conformational statistics. 
We have derived two techniques-field-theoretic umbrella sampling [21] and forcematching - to construct the excess free-energy functional from a particle-based model. Since on the large time and length scale of interest, the relevant free-energy scale is comparable to or smaller than the thermal energy scale, $k_{B} T$, a bottom-up construction of the coarsegrained interactions from an atomistic description remains a formidable challenge. Nevertheless we expect that these technique will be useful for relating a particle-based model to a continuum field-theoretic description, where the collective densities are the relevant degrees of freedom.

Then we have discussed computational strategies for calculating the free energy of selfassembled structures by computer simulations. The relation between simulation techniques for hard crystalline matter and soft self-assembled structures has been illustrated and strategies to transfer methods from hard crystals to soft self-assembled structures have been discussed. Alternatively, one can exploit the ability of accurately and simultaneously measuring the chemical potential and pressure to assess the thermodynamic stability of self-assembled morphologies [32]. These techniques are important because the free-energy difference between self-assembled morphologies is small on a per molecule basis and metastable structures may persist for a very long time. Accurate and computational efficient strategies are required for establishing equilibrium properties in the bulk, of defects and of interfaces or grain boundaries. Given the multitude of molecular architectures and equilibrium morphologies in block copolymer materials in the bulk (e.g., that are formed by $A B C$ triblock copolymers [215]) and the even larger number of defect and interfacial structures, the exploration of the thermodynamic properties of self-assembled systems remains a computational challenge.

By virtue of the small free-energy difference of competing bulk morphologies or defects, the thermodynamic driving forces in soft matter are weak and metastable structures have a protracted lifetime. Thus it is important to understand and control the kinetics of structure formation, e.g., in order to avoid the formation of defects or in order to purposely generate a metastable morphology. To make progress, one needs to know the underlying free-energy landscape of the metastable morphologies and their transition states as well as the relation between the molecular motion and the kinetics of the collective composition or density fields. Advances have been achieved in various aspects, e.g., transition-path sampling for self-assembled systems within the self-consistent field theory [197], but many challenges, e.g., relaxation of molecular stress/break-down of the assumption that the molecular conformations are in equilibrium with the instantaneous density [216], remain.

Acknowledgements It is a great pleasure to thank Kostas Ch. Daoulas, Francois A. Detcheverry, Martin Hömberg, Paul F. Nealey, Juan J. de Pablo, Darin Q. Pike, Abelardo Ramirez-Hernandez, and Birger Steinmüller, for fruitful and enjoyable collaboration, stimulating discussions, and critical reading of the manuscript. Financial support has been provided by the Volkswagen foundation and the Deutsche Forschungsgemeinschaft (DFG) under grant No. Mu1674/11.

Open Access This article is distributed under the terms of the Creative Commons Attribution Noncommercial License which permits any noncommercial use, distribution, and reproduction in any medium, provided the original author(s) and source are credited.

\section{Appendix: Field-Theoretic Derivation of the Force-Matching Relation, (50)}

Using the Fourier representation of the $\delta$-function constraint, we can rewrite (8) in the form

$$
\mathcal{S}=k_{B} \ln \int \mathcal{D}\left[\left\{\mathbf{r}_{i, s}\right\}\right] e^{-\frac{\mathcal{H}_{\mathrm{b}}\left(\left\{\mathbf{r}_{i, s}\right)\right)}{k_{B} T}} \delta\left(\phi_{A}-\hat{\phi}_{A}\right) \delta\left(\phi_{B}-\hat{\phi}_{B}\right)
$$




$$
=k_{B} \ln \int \mathcal{D}\left[w_{A}, w_{B}\right] \mathcal{D}\left[\left\{\mathbf{r}_{i, s}\right\}\right] e^{-\frac{\mathcal{H}_{\mathrm{b}}\left(\left\{\mathbf{r}_{i, s}\right\}\right)}{k_{B} T}} e^{\frac{\rho_{0}}{k_{B} T} \int \mathrm{d}^{3} \mathbf{r}\left[w_{A}\left(\phi_{A}-\hat{\phi}_{A}\right)+w_{B}\left(\phi_{B}-\hat{\phi}_{B}\right)\right]}
$$

where $w_{A}$ and $w_{B}$ are complex auxiliary fields. In this framework the chemical potentials are given by averages of these complex-valued fluctuating fields

$$
\mu_{A}(\mathbf{r})=-T \frac{\delta \mathcal{S}}{\delta \phi_{A}(\mathbf{r})}=-\rho_{\mathrm{o}}\left\langle w_{A}(\mathbf{r})\right\rangle_{\phi_{A}, \phi_{B}}
$$

The constraint average $\langle\cdots\rangle_{\phi_{A}, \phi_{B}}$ is defined by

$$
\begin{aligned}
& \langle\cdots\rangle_{\phi_{A}, \phi_{B}} \\
& =\frac{\int \mathcal{D}\left[w_{A}, w_{B}\right] \mathcal{D}\left[\left\{\mathbf{r}_{i, s}\right\}\right] e^{\frac{\rho_{0}}{k_{B} T} \int \mathrm{d}^{3} \mathbf{r}\left[w_{A} \phi_{A}+w_{B} \phi_{B}\right]-\frac{\mathcal{H}_{\mathrm{b}}\left(\left\{\mathbf{r}_{i, s}\right)+\sum_{i, s}\left[\gamma_{i, s} w_{A}\left(\mathbf{r}_{i, s}\right)+\left(1-\gamma_{i, s}\right) w_{B}\left(\mathbf{r}_{i, s}\right)\right]\right.}{k_{B} T} \ldots} \cdots}{\int \mathcal{D}\left[w_{A}, w_{B}\right] \mathcal{D}\left[\left\{\mathbf{r}_{i, s}\right\}\right] e^{\frac{\rho_{0}}{k_{B} T} \int \mathrm{d}^{3} \mathbf{r}\left[w_{A} \phi_{A}+w_{B} \phi_{B}\right]-\frac{\mathcal{H}_{\mathrm{b}}\left(\left\{\mathbf{r}_{i, s}\right\}\right)+\sum_{i, s}\left[\gamma_{i, s} w_{A}\left(\mathbf{r}_{i, s}\right)+\left(1-\gamma_{i, s}\right) w_{B}\left(\mathbf{r}_{i, s}\right)\right]}{k_{B} T}}} \\
& =\frac{\int \mathcal{D}\left[w_{A}, w_{B}\right] e^{\frac{\rho_{0}}{k_{B} T} \int \mathrm{d}^{3} \mathbf{r}\left[w_{A} \phi_{A}+w_{B} \phi_{B}\right]+\ln \mathcal{Z}\left[w_{A}, w_{B}\right]}\langle\cdots\rangle_{w_{A}, w_{B}}}{\int \mathcal{D}\left[w_{A}, w_{B}\right] e^{\frac{\rho_{0}}{k_{B} T} \int \mathrm{d}^{3} \mathbf{r}\left[w_{A} \phi_{A}+w_{B} \phi_{B}\right]+\ln \mathcal{Z}\left[w_{A}, w_{B}\right]}} \\
& =e^{-\mathcal{S} / k_{B}} \int \mathcal{D}\left[w_{A}, w_{B}\right] e^{\frac{\rho_{0}}{k_{B} T} \int \mathrm{d}^{3} \mathbf{r}\left[w_{A} \phi_{A}+w_{B} \phi_{B}\right]+\ln \mathcal{Z}\left[w_{A}, w_{B}\right]}\langle\cdots\rangle_{w_{A}, w_{B}} \\
& \equiv\left[\langle\cdots\rangle_{w_{A}, w_{B}}\right]_{\phi_{A}, \phi_{B}}
\end{aligned}
$$

where $\langle\cdots\rangle_{w_{A}, w_{B}}$ denotes the average of the unconstraint system subjected to the external fields, $w_{A}$ and $w_{B}$, and $\mathcal{Z}\left[w_{A}, w_{B}\right]$ is the canonical partition function of the unconstraint particle system in the auxiliary fields.

$$
\mathcal{Z}\left[w_{A}, w_{B}\right]=\int \mathcal{D}\left[\left\{\mathbf{r}_{i, s}\right\}\right] e^{-\frac{\mathcal{H}_{\mathrm{b}}\left(\left\{\mathbf{r}_{i, s}\right\}\right)+\sum_{i, s}\left[\gamma_{i, s} w_{A}\left(\mathbf{r}_{i, s}\right)+\left(1-\gamma_{i, s}\right) w_{B}\left(\mathbf{r}_{i, s}\right)\right]}{k_{B} T}}
$$

In order to derive a force-balance relation, we first consider the unconstraint system in the external fields, $w_{A}$ and $w_{B}$. The density of the $A$ segment with index $j, t$ is given by ${ }^{6}$

$$
\begin{aligned}
\rho_{\mathrm{o}}\left\langle\hat{\phi}_{A j, t}(\mathbf{r})\right\rangle_{w_{A}, w_{B}} & \equiv\left\langle\delta\left(\mathbf{r}-\mathbf{r}_{i, t}\right)\right\rangle_{w_{A}, w_{B}} \\
& =\frac{1}{\mathcal{Z}\left[w_{A}, w_{B}\right]} \int \mathcal{D}^{\prime}\left[\left\{\mathbf{r}_{i, s}\right\}\right] e^{-\frac{\mathcal{H}_{\mathrm{b}}\left(\left\{\mathbf{r}_{i, s}\right\}\right)+\sum_{i, s}\left[\gamma_{i, s} w_{A}\left(\mathbf{r}_{i, s}\right)+\left(1-\gamma_{i, s}\right) w_{B}\left(\mathbf{r}_{i, s}\right)\right]}{k_{B} T}}
\end{aligned}
$$

where the prime indicates that the configurational integral is only taken over the $n N-1$ other segments but not the $A$ segment $j, t$. The differentiation of this relation with respect to the segment position, $\mathbf{r}$ yields

\footnotetext{
${ }^{6}$ The force balance for the unconstraint system in the fixed external fields, $w_{A}$ and $w_{B}$, is described by the lowest-order equation of the Born-Green-Yvon hierarchy [44, 112, 113].

$$
\nabla\left[k_{B} T \ln \left\langle\hat{\phi}_{A}(\mathbf{r})\right\rangle_{w_{A}, w_{B}}+w_{A}(\mathbf{r})\right]-\left\langle\mathbf{K}_{A}(\mathbf{r})\right\rangle_{w_{A}, w_{B}}=0
$$

where the spatial variation of the local density of states, $k_{B} T \ln \left\langle\hat{\phi}_{A}\right\rangle_{w_{A}, w_{B}}$, is balanced by the force of the external field and the average force, $\left\langle\mathbf{K}_{A}(\mathbf{r})\right\rangle_{w_{A}, w_{B}}$, with the neighboring particles [217, 218]. In the following we generalize the derivation of this force-balance relation for a constraint system.
} 


$$
\begin{aligned}
\rho_{\mathrm{o}} \nabla\left\langle\hat{\phi}_{A j, t}(\mathbf{r})\right\rangle_{w_{A}, w_{B}}= & \frac{1}{\mathcal{Z}\left[w_{A}, w_{B}\right]} \int \mathcal{D}^{\prime}\left[\left\{\mathbf{r}_{i, s}\right\}\right] e^{-\frac{\mathcal{H}_{\mathrm{b}}\left(\left\{\mathbf{r}_{i, s}\right\}\right)+\sum_{i, s}\left[\gamma_{i, s} w_{A}\left(\mathbf{r}_{i, s}\right)+\left(1-\gamma_{i, s}\right) w_{B}\left(\mathbf{r}_{i, s}\right)\right]}{k_{B} T}} \\
& \times \frac{-\left.\nabla_{j, t} \mathcal{H}_{\mathrm{b}}\left(\left\{\mathbf{r}_{i, s}\right\}\right)\right|_{\mathbf{r}=\mathbf{r}_{j, t}}-\nabla w_{A}(\mathbf{r})}{k_{B} T} \\
= & -\frac{\rho_{\mathrm{o}}}{k_{B} T}\left(\left\langle\left.\hat{\phi}_{A j, t}(\mathbf{r}) \nabla_{j, t} \mathcal{H}_{\mathrm{b}}\left(\left\{\mathbf{r}_{i, s}\right\}\right)\right|_{\left.\mathbf{r}=\mathbf{r}_{j, t}\right\rangle_{w_{A}, w_{B}}}\right.\right. \\
& \left.+\left\langle\hat{\phi}_{A j, t}(\mathbf{r})\right\rangle_{w_{A}, w_{B}} \nabla w_{A}(\mathbf{r})\right)
\end{aligned}
$$

Averaging both sides of the equation with respect to the fluctuating fields, $w_{A}$ and $w_{B}$, and summing over all $A$ segments, one obtains

$$
\begin{aligned}
\rho_{\mathrm{o}} \nabla\left[\left\langle\hat{\phi}_{A}(\mathbf{r})\right\rangle_{w_{A}, w_{B}}\right]_{\phi_{A}, \phi_{B}}= & \rho_{\mathrm{o}} \nabla\left\langle\hat{\phi}_{A}(\mathbf{r})\right\rangle_{\phi_{A}, \phi_{B}}=\rho_{\mathrm{o}} \nabla \phi_{A}(\mathbf{r}) \\
= & -\frac{\rho_{\mathrm{o}}}{k_{B} T} \sum_{j, t} \gamma_{j, t}\left\langle\left.\hat{\phi}_{A j, t}(\mathbf{r}) \nabla_{j, t} \mathcal{H}_{\mathrm{b}}\left(\left\{\mathbf{r}_{i, s}\right\}\right)\right|_{\mathbf{r}=\mathbf{r}_{j, t}}\right\rangle_{\phi_{A}, \phi_{B}} \\
& -\frac{\rho_{\mathrm{o}}}{k_{B} T} \sum_{j, t} \gamma_{j, t}\left\langle\hat{\phi}_{A j, t}(\mathbf{r}) \nabla w_{A}(\mathbf{r})\right\rangle_{\phi_{A}, \phi_{B}} \\
= & +\frac{\rho_{\mathrm{o}} \phi_{A}(\mathbf{r})}{k_{B} T}\left\langle\mathbf{K}_{A}(\mathbf{r})\right\rangle_{\phi_{A}, \phi_{B}} \\
& -\frac{\rho_{\mathrm{o}}}{k_{B} T}\left[\left\langle\hat{\phi}_{A}(\mathbf{r})\right\rangle_{w_{A}, w_{B}} \nabla w_{A}(\mathbf{r})\right]_{\phi_{A}, \phi_{B}}
\end{aligned}
$$

where

$$
\left\langle\mathbf{K}_{A}(\mathbf{r})\right\rangle_{\phi_{A}, \phi_{B}}=-\frac{\sum_{j, t} \gamma_{j, t}\left\langle\left.\hat{\phi}_{A j, t}(\mathbf{r}) \nabla_{j, t} \mathcal{H}_{\mathrm{b}}\left(\left\{\mathbf{r}_{i, s}\right\}\right)\right|_{\mathbf{r}=\mathbf{r}_{j, t}}\right\rangle_{\phi_{A}, \phi_{B}}}{\sum_{j, t} \gamma_{j, t}\left\langle\hat{\phi}_{A j, t}(\mathbf{r})\right\rangle_{\phi_{A}, \phi_{B}}}
$$

is the force acting on $A$ segments at position $\mathbf{r}$ in the constraint system.

Using

$$
\left\langle\hat{\phi}_{A}(\mathbf{r})\right\rangle_{w_{A}, w_{B}}=-\frac{k_{B} T}{\rho_{\mathrm{o}}} \frac{\delta}{\delta w_{A}(\mathbf{r})} \ln \mathcal{Z}\left[w_{A}, w_{B}\right]
$$

we can rewrite the last term of (137) in the form

$$
\begin{aligned}
& {\left[\left\langle\hat{\phi}_{A}(\mathbf{r})\right\rangle_{w_{A}, w_{B}} \nabla w_{A}(\mathbf{r})\right]_{\phi_{A}, \phi_{B}}} \\
& \quad=e^{-\mathcal{S} / k_{B}} \int \mathcal{D}\left[w_{A}, w_{B}\right] e^{\frac{\rho_{0}}{k_{B} T} \int \mathrm{d} \mathbf{r}\left[w_{A} \phi_{A}+w_{B} \phi_{B}\right]+\ln \mathcal{Z}}\left(-\frac{k_{B} T}{\rho_{\mathrm{o}}} \frac{\delta \ln \mathcal{Z}}{\delta w_{A}(\mathbf{r})}\right) \nabla w_{A}(\mathbf{r}) \\
& \quad=e^{-\mathcal{S} / k_{B}} \int \mathcal{D}\left[w_{A}, w_{B}\right] e^{\frac{\rho_{0}}{k_{B} T} \int \mathrm{d} \mathbf{r}\left[w_{A} \phi_{A}+w_{B} \phi_{B}\right]}\left(-\frac{k_{B} T}{\rho_{\mathrm{o}}} \frac{\delta \mathcal{Z}}{\delta w_{A}(\mathbf{r})}\right) \nabla w_{A}(\mathbf{r}) \\
& =e^{-\mathcal{S} / k_{B}} \frac{k_{B} T}{\rho_{\mathrm{o}}} \int \mathcal{D}\left[w_{A}, w_{B}\right] \mathcal{Z} \frac{\delta}{\delta w_{A}(\mathbf{r})}\left(e^{\frac{\rho_{0}}{k_{B} T} \int \mathrm{d} \mathbf{r}\left[w_{A} \phi_{A}+w_{B} \phi_{B}\right]} \nabla w_{A}(\mathbf{r})\right) \\
& \quad=+e^{-\mathcal{S} / k_{B}} \int \mathcal{D}\left[w_{A}, w_{B}\right] \mathcal{Z} \phi_{A}(\mathbf{r}) e^{\frac{\rho_{0}}{k_{B} T} \int \mathrm{d} \mathbf{r}\left[w_{A} \phi_{A}+w_{B} \phi_{B}\right]} \nabla w_{A}(\mathbf{r})
\end{aligned}
$$




$$
\begin{aligned}
& +e^{-\mathcal{S} / k_{B}} \frac{k_{B} T}{\rho_{\mathrm{o}}} \int \mathcal{D}\left[w_{A}, w_{B}\right] \mathcal{Z} e^{\frac{\rho_{0}}{k_{B} T} \int \mathrm{d}\left[w_{A} \phi_{A}+w_{B} \phi_{B}\right]} \underbrace{\frac{\delta \nabla w_{A}(\mathbf{r})}{\delta w_{A}(\mathbf{r})}}_{=0} \\
= & \phi_{A}(\mathbf{r}) \nabla e^{-\mathcal{S} / k_{B}} \int \mathcal{D}\left[w_{A}, w_{B}\right] e^{\frac{\rho_{0}}{k_{B} T} \int \mathrm{d} \mathbf{r}\left[w_{A} \phi_{A}+w_{B} \phi_{B}\right]+\ln \mathcal{Z}} w_{A}(\mathbf{r}) \\
= & \phi_{A}(\mathbf{r}) \nabla\left[w_{A}(\mathbf{r})\right]_{\phi_{A}, \phi_{B}}=\phi_{A}(\mathbf{r}) \nabla\left\langle w_{A}(\mathbf{r})\right\rangle_{\phi_{A}, \phi_{B}}
\end{aligned}
$$

Inserting this result in (137), we find

$$
\nabla\left[k_{B} T \ln \phi_{A}(\mathbf{r})+\left\langle w_{A}(\mathbf{r})\right\rangle_{\phi_{A}, \phi_{B}}\right]-\left\langle\mathbf{K}_{A}(\mathbf{r})\right\rangle_{\phi_{A}, \phi_{B}}=0
$$

which, in conjunction with (125), yields the force-balance relation, (50).

\section{References}

1. Seul, M., Andelman, D.: Science 267, 476 (1995)

2. Shelley, J.C., Shelley, M., Reeder, R., Bandyopadhay, S., Klein, M.: J. Phys. Chem. B 105, 4464 (2001)

3. Ayton, G., Voth, G.: Biophys. J. 83, 3357 (2002)

4. Müller, M., Katsov, K., Schick, M.: J. Polym. Sci., Part B, Polym. Phys. 41, 1441 (2003)

5. Izvekov, S., Voth, G.A.: J. Phys. Chem. B 109, 2469 (2005)

6. Müller, M., Katsov, K., Schick, M.: Phys. Rep. 434, 113 (2006)

7. Ayton, G.S., Noid, W.G., Voth, G.A.: Curr. Opin. Struct. Biol. 17, 192 (2007)

8. Venturoli, M., Sperotto, M.M., Kranenburg, M., Smit, B.: Phys. Rep. 437, 1 (2006)

9. Marrink, S.J., Risselada, H.J., Yefimov, S., Tieleman, D.P., de Vries, A.H.: J. Phys. Chem. B 111, 7812 (2007)

10. Murtola, T., Bunker, A., Vattulainen, I., Deserno, M., Karttunen, M.: Phys. Chem. Chem. Phys. 11, 1869 (2009)

11. Daoulas, K.C., Müller, M.: Adv. Polym. Sci. 224, 197 (2010)

12. Groot, R.D., Warren, P.B.: J. Chem. Phys. 107, 4423 (1997)

13. Groot, R.D., Madden, T.J.: J. Chem. Phys. 108, 8713 (1998)

14. Groot, R.D., Madden, T.J., Tildesley, D.J.: J. Chem. Phys. 110, 9739 (1999)

15. Müller, M., Smith, G.D.: J. Polym. Sci., Part B, Polym. Phys. 43, 934 (2005)

16. Daoulas, K.C., Müller, M., de Pablo, J.J., Nealey, P.F., Smith, G.D.: Soft Matter 2, 573 (2006)

17. Detcheverry, F.A., Kang, H.M., Daoulas, K.C., Müller, M., Nealey, P.F., de Pablo, J.J.: Macromolecules 41, 4989 (2008)

18. Detcheverry, F.A., Pike, D.Q., Nealey, P.F., Müller, M., de Pablo, J.J.: Phys. Rev. Lett. 102, 197801 (2009)

19. Wang, Q., Yin, Y.: J. Chem. Phys. 130, 104903 (2009)

20. Wang, Q.: Soft Matter 5, 4564 (2009)

21. Müller, M.: Eur. Phys. J. Special Topics 177, 149 (2009)

22. Hömberg, M., Müller, M.: J. Chem. Phys. 132, 155104 (2010)

23. Wang, Z.J., Deserno, M.: New J. Phys. 12, 095004 (2010)

24. Lyubartsev, A.P., Rabinovich, A.L.: New J. Phys. 7, 25 (2011)

25. Daoulas, K.C., Müller, M.: J. Chem. Phys. 125, 184904 (2006)

26. Müller, M., Daoulas, K.C.: J. Chem. Phys. 128, 024903 (2008)

27. Müller, M., Daoulas, K.C.: J. Chem. Phys. 129, 499 (2008)

28. Müller, M., Daoulas, K.C., Norizoe, Y.: Phys. Chem. Chem. Phys. 11, 2087 (2009)

29. Pike, D.Q., Detcheverry, F.A., Müller, M., de Pablo, J.J.: J. Chem. Phys. 131 (2009)

30. Norizoe, Y., Daoulas, K.C., Müller, M.: Faraday Discussions 144, 369 (2010)

31. Detcheverry, F.A., Pike, D.Q., Nealey, P.F., Müller, M., de Pablo, J.J.: Faraday Discussions 144, 111 (2010)

32. Escobedo, F.A.: J. Chem. Phys. 123, 044110 (2005)

33. Martinez-Veracoechea, F.J., Escobedo, F.A.: J. Chem. Phys. 125, 104907 (2006)

34. Lennon, E.M., Katsov, K., Fredrickson, G.H.: Phys. Rev. Lett. 101, 138302 (2008)

35. Israelachvili, J.N.: Intermolecular and Surface Forces, 2nd edn. Academic Press, London (1991)

36. Bates, F.S., Fredrickson, G.H.: Annu. Rev. Phys. Chem. 41, 525 (1990) 
37. Matsen, M.W., Bates, F.S.: Macromolecules 29, 1091 (1996)

38. Matsen, M.W., Bates, F.S.: Macromolecules 29, 7641 (1996)

39. Baschnagel, J., Binder, K., Doruker, P., Gusev, A.A., Hahn, O., Kremer, K., Mattice, W.L., MüllerPlathe, F., Murat, M., Paul, W., Santos, S., Suter, U.W., Tries, V.: Adv. Polym. Sci. 152, 41 (2000)

40. Müller-Plathe, F.: Chem. Phys. Chem. 3, 754 (2002)

41. Kremer, K., Müller-Plathe, F.: Mol. Simul. 28, 729 (2002)

42. Praprotnik, M., delle Site, L., Kremer, K.: Annu. Rev. Phys. Chem. 59, 545 (2008)

43. Noid, W.G., Chu, J.W., Ayton, G.S., Krishna, V., Izvekov, S., Voth, G.A., Das, A., Andersen, H.C.: J. Chem. Phys. 128, 3232 (2008)

44. Mullinax, J.W., Noid, W.G.: Phys. Rev. Lett. 103, 198104 (2009)

45. Wilson, K.G.: Phys. Rev. B 4, 3174 (1971)

46. Ma, S.K.: Phys. Rev. Lett. 37, 461 (1976)

47. Swendsen, R.H.: Phys. Rev. Lett. 42, 859 (1979)

48. Binder, K.: Phys. Rev. Lett. 47, 693 (1981)

49. de Gennes, P.G.: Phys. Lett. A 38, 339 (1972)

50. Schäfer, L.: Excluded Volume Effects in Polymer Solutions, as Explained by the Renormalization Group. Springer, Berlin/New York (1999)

51. Bolhuis, P.G., Louis, A.A., Hansen, J.P.: Phys. Rev. E 6402, 021801 (2001)

52. Louis, A.A.: J. Phys., Condens. Matter 14, 9187 (2002)

53. Krishna, V., Noid, W.G., Voth, G.A.: J. Chem. Phys. 131, 2002 (2009)

54. Forrest, B.M., Suter, U.W.: J. Chem. Phys. 102, 7256 (1995)

55. Louis, A.A., Bolhuis, P.G., Hansen, J.P., Meijer, E.J.: Phys. Rev. Lett. 85, 2522 (2000)

56. Helfand, E.: J. Chem. Phys. 62, 999 (1975)

57. Müller, M., Schmid, F.: Adv. Polym. Sci. 185, 1 (2005)

58. Sariban, A., Binder, K.: Macromolecules 21, 711 (1988)

59. Müller, M.: Macromol. Theory Simul. 8, 343 (1999)

60. Wang, Z.G.: J. Chem. Phys. 117, 481 (2002)

61. Grzywacz, P., Qin, J., Morse, D.C.: Phys. Rev. E 76, 061802 (2007)

62. Qin, J., Morse, D.C.: J. Chem. Phys. 130, 224902 (2009)

63. Morse, D.C., Chung, J.K.: J. Chem. Phys. 130 (2009)

64. Fredrickson, G.H., Helfand, E.: J. Chem. Phys. 87, 697 (1987)

65. Morse, D.C.: Ann. Phys. 321, 2318 (2006)

66. Werner, A., Schmid, F., Müller, M., Binder, K.: Phys. Rev. E 59, 728 (1999)

67. Müller, M., MacDowell, L.G.: J. Phys., Condens. Matter 15, R609 (2003)

68. Müller, M., Binder, K.: J. Phys., Condens. Matter 17, S333 (2005)

69. Fuchs, M., Müller, M.: Phys. Rev. E 60, 1921 (1999)

70. Müller, M., Binder, K., Schäfer, L.: Macromolecules 33, 4568 (2000)

71. Wittmer, J.P., Meyer, H., Baschnagel, J., Johner, A., Obukhov, S., Mattioni, L., Müller, M., Semenov, A.N.: Phys. Rev. Lett. 93, 147801 (2004)

72. Cavallo, A., Müller, M., Wittmer, J.P., Johner, A., Binder, K.: J. Phys., Condens. Matter 17, S1697 (2005)

73. Cavallo, A., Müller, M., Binder, K.: J. Phys. Chem. B 109, 6544 (2005)

74. Wittmer, J.P., Beckrich, P., Meyer, H., Cavallo, A., Johner, A., Baschnagel, J.: Phys. Rev. E 76, 4937 (2007)

75. Beckrich, P., Johner, A., Semenov, A.N., Obukhov, S.P., Benoit, H., Wittmer, J.P.: Macromolecules 40, 3805 (2007)

76. Wittmer, J.P., Cavallo, A., Kreer, T., Baschnagel, J., Johner, A.: J. Chem. Phys. 131 (2009)

77. Wittmer, J.P., Cavallo, A., Xu, H., Zabel, J.E., Polinska, P., Schulmann, N., Meyer, H., Farago, J., Johner, A., Obukhov, S.P., Baschnagel, J.: J. Stat. Phys. (2011, in press). arXiv:1107.4454v1

78. Wittmer, J.P., Polinska, P., Meyer, H., Farago, J., Johner, A., Baschnagel, J., Cavallo, A.: J. Chem. Phys. 134, 234901 (2011)

79. Flory, P.J.: J. Chem. Phys. 9, 660 (1941)

80. Huggins, M.L.: J. Chem. Phys. 9, 440 (1941)

81. Carmesin, I., Kremer, K.: Macromolecules 21, 2819 (1988)

82. Deutsch, H.P., Binder, K.: J. Chem. Phys. 94, 2294 (1991)

83. Müller, M., Paul, W.: J. Chem. Phys. 100, 719 (1994)

84. Grest, G.S., Kremer, K.: Phys. Rev. A 33, 3628 (1986)

85. Murat, M., Grest, G.S., Kremer, K.: Macromolecules 32, 595 (1999)

86. de Gennes, P.G.: J. Chem. Phys. 55, 572 (1971)

87. Doi, M., Edwards, S.: The Theory of Polymer Dynamics. Oxford University Press, New York (1994)

88. Edwards, S.F.: Proc. Phys. Soc. 85, 613 (1965) 
89. Reith, D., Pütz, M., Müller-Plathe, F.: J. Comput. Chem. 24, 1624 (2003)

90. Ercolessi, F., Adams, J.B.: Europhys. Lett. 26, 583 (1994)

91. Izvekov, S., Parrinello, M., Burnham, C.J., Voth, G.A.: J. Chem. Phys. 120, 10896 (2004)

92. Edwards, E.W., Stoykovich, M.P., Müller, M., Solak, H.H., de Pablo, J.J., Nealey, P.F.: J. Polym. Sci., Part B, Polym. Phys. 43, 3444 (2005)

93. Daoulas, K.C., Müller, M., Stoykovich, M.P., Papakonstantopoulos, Y.J., de Pablo, J.J., Nealey, P.F., Park, S.M., Solak, H.H.: J. Polym. Sci., Part B, Polym. Phys. 44, 2589 (2006)

94. Müller, M., MacDowell, L.G., Virnau, P., Binder, K.: J. Chem. Phys. 117, 5480 (2002)

95. Müller, M.: Phys. Rev. E 65, 030802 (2002)

96. Hong, K.M., Noolandi, J.: Macromolecules 14, 727 (1981)

97. Laradji, M., Guo, H., Zuckermann, M.J.: Phys. Rev. E 49, 3199 (1994)

98. Lifshitz, I.M., Grosberg, A.Y., Khokhlov, A.R.: Rev. Mod. Phys. 50, 683 (1978)

99. de Gennes, P.G.: Scaling Concepts in Polymer Physics. Cornell University Press, Ithaca (1979)

100. Leibler, L.: Macromolecules 13, 1602 (1980)

101. Ohta, T., Kawasaki, K.: Macromolecules 19, 2621 (1986)

102. de la Cruz, M.O., Sanchez, I.C.: Macromolecules 19, 2501 (1986)

103. Fredrickson, G.H., Milner, S.T., Leibler, L.: Macromolecules 25, 6341 (1992)

104. Werner, A., Fredrickson, G.H.: J. Polym. Sci., Part B, Polym. Phys. 35, 849 (1997)

105. Shinozaki, A., Jasnow, D., Balazs, A.C.: Macromolecules 27, 2496 (1994)

106. Cochran, E.W., Morse, D.C., Bates, F.S.: Macromolecules 36, 782 (2003)

107. Erukhimovich, I.Y.: Eur. Phys. J. E 18, 383 (2005)

108. Palyulin, V.V., Potemkin, I.I.: J. Chem. Phys. 127, 124903 (2007)

109. Aliev, M.A., Kuchanov, S.I.: J. Chem. Phys. 131, 174111 (2009)

110. van der Heydt, A., Müller, M., Zippelius, A.: Macromolecules 43, 3161 (2010)

111. Villet, M.C., Fredrickson, G.H.: J. Chem. Phys. 132, 034109 (2010)

112. Barker, J.A., Henderson, D.: Rev. Mod. Phys. 48, 587 (1976)

113. Hansen, J.P., McDonald, I.R.: Theory of Simple Liquids. Academic Press, London (1986)

114. Mavrantzas, V.G., Boone, T.D., Zervopoulou, E., Theodorou, D.N.: Macromolecules 32, 5072 (1999)

115. Maragliano, L., Vanden-Eijnden, E.: Chem. Phys. Lett. 426, 168 (2006)

116. Kevrekidis, I.G., Gear, C.W., Hummer, G.: AIChE J. 50, 1346 (2004)

117. E, W., Engquist, B., Li, X.T., Ren, W.Q., Vanden-Eijnden, E.: Commun. Comput. Phys. 2, 367 (2007)

118. Vanden-Eijnden, E.: Commun. Math. Sci. 5, 495 (2007)

119. E, W., Ren, W.Q., Vanden-Eijnden, E.: J. Comput. Phys. 228, 5437 (2009)

120. Helfand, E., Tagami, Y.: J. Polym. Sci., Part B, Polym. Lett. 9, 741 (1971)

121. Wu, D.T., Fredrickson, G.H., Carton, J.P., Adjdari, A., Leibler, L.: J. Polym. Sci., Part B, Polym. Phys. 33, 2373 (1995)

122. Matsen, M.W.: J. Phys., Condens. Matter 14, R21 (2002)

123. Wu, J.Z.: AIChE J. 52, 1169 (2006)

124. Wu, J.Z., Li, Z.D.: Annu. Rev. Phys. Chem. 58, 85 (2007)

125. Wang, J., Müller, M.: Macromolecules 42, 2251 (2009)

126. Wang, J.F., Müller, M.: Langmuir 26, 1291 (2010)

127. Curtin, W.A., Ashcroft, N.W.: Phys. Rev. A 32, 2909 (1985)

128. Eastwood, J.W., Hockney, R.W., Lawrence, D.N.: Comput. Phys. Commun. 19, 215 (1980)

129. Dawson, J.M.: Rev. Mod. Phys. 55, 403 (1983)

130. Müller, M., Pastorino, C.: Europhys. Lett. 81, 28002 (2008)

131. Dickman, R.: J. Chem. Phys. 91, 454 (1989)

132. Ivanov, V.A., An, E.A., Spirin, L.A., Stukan, M.R., Müller, M., Paul, W., Binder, K.: Phys. Rev. E 76, 026702 (2007)

133. Miao, L., Guo, H., Zuckermann, M.J.: Macromolecules 29, 2289 (1996)

134. Likos, C.N., Mladek, B.M., Gottwald, D., Kahl, G.: J. Chem. Phys. 126, 224502 (2007)

135. Pagonabarraga, I., Frenkel, D.: J. Chem. Phys. 115, 5015 (2001)

136. Trofimov, S.Y., Nies, E.L.F., Michels, M.A.J.: J. Chem. Phys. 117, 9383 (2002)

137. Wang, Q.: Soft Matter 7, 3711 (2011)

138. Ramirez-Hernandez, A., Detcheverry, F.A., de Pablo, J.J.: J. Chem. Phys. 133, 64905 (2010)

139. Müller, M., Steinmüller, B., Daoulas, K.C., Ramirez-Hernandez, A., de Pablo, J.: Phys. Chem. Chem. Phys. 13, 10491 (2011)

140. Padding, J.T., Briels, W.J.: J. Chem. Phys. 115, 2846 (2001)

141. Padding, J.T., Briels, W.J.: J. Chem. Phys. 117, 925 (2002)

142. Hua, C.C., Schieber, J.D.: J. Chem. Phys. 109, 10018 (1998)

143. Schieber, J.D., Neergaard, J., Gupta, S.: J. Rheol. 47, 213 (2003)

144. Nair, D.M., Schieber, J.D.: Macromolecules 39, 3386 (2006) 
145. Schieber, J.D., Nair, D.M., Kitkrailard, T.: J. Rheol. 51, 1111 (2007)

146. Likhtman, A.E.: Macromolecules 38, 6128 (2005)

147. Ramirez, J., Sukumaran, S.K., Likhtman, A.E.: J. Chem. Phys. 126, 244904 (2007)

148. Müller, M., MacDowell, L.G., Müller-Buschbaum, P., Wunnike, O., Stamm, M.: J. Chem. Phys. 115, 9960 (2001)

149. Müller, M., Daoulas, K.C.: J. Chem. Phys. 129, 164906 (2008)

150. Muller, M., Wittmer, J.P., Cates, M.E.: Phys. Rev. E 53, 5063 (1996)

151. Brown, S., Szamel, G.: J. Chem. Phys. 109, 6184 (1998)

152. Müller, M., Wittmer, J.P., Cates, M.E.: Phys. Rev. E 61, 4078 (2000)

153. Vettorel, T., Grosberg, A.Y., Kremer, K.: Phys. Biol. 6, 025013 (2009)

154. Fetters, L.J., Lohse, D.J., Milner, S.T., Graessley, W.W.: Macromolecules 32, 6847 (1999)

155. Fetters, L.J., Lohse, D.J., Graessley, W.W.: J. Polym. Sci., Part B, Polym. Phys. 37, 1023 (1999)

156. Kremer, K., Grest, G.S.: J. Chem. Phys. 92, 5057 (1990)

157. Everaers, R., Sukumaran, S.K., Grest, G.S., Svaneborg, C., Sivasubramanian, A., Kremer, K.: Science 303, 823 (2004)

158. Rouse, P.E.: J. Chem. Phys. 21, 1272 (1953)

159. Dotera, T., Hatano, A.: J. Chem. Phys. 105, 8431 (1996)

160. Dalvi, M.C., Eastman, C.E., Lodge, T.P.: Phys. Rev. Lett. 71, 2591 (1993)

161. Dalvi, M.C., Lodge, T.P.: Macromolecules 26, 859 (1993)

162. Lodge, T.P., Dalvi, M.C.: Phys. Rev. Lett. 75, 657 (1995)

163. Fredrickson, G.H., Bates, F.S.: Annu. Rev. Mater. Sci. 26, 501 (1996)

164. Barrat, J.L., Fredrickson, G.H.: Macromolecules 24, 6378 (1991)

165. Ramanathan, S., Morse, D.C.: J. Chem. Phys. 126, 094906 (2007)

166. Rubinstein, M., Panyukov, S.: Macromolecules 30, 8036 (1997)

167. Rubinstein, M., Panyukov, S.: Macromolecules 35, 6670 (2002)

168. Rossky, P.J., Doll, J.D., Friedman, H.L.: J. Chem. Phys. 69, 4628 (1978)

169. Pütz, M., Kremer, K., Grest, G.S.: Europhys. Lett. 49, 735 (2000)

170. Kron, A.K.: Polymer. Sci. USSR 7, 1361 (1965)

171. Wall, F.T., Mandel, F.: J. Chem. Phys. 63, 4592 (1975)

172. Leonforte, F.: Phys. Rev. E 82, 041802 (2010)

173. Vladkov, M., Barrat, J.L.: Macromolecules 40, 3797 (2007)

174. Hoy, R.S., Grest, G.S.: Macromolecules 40, 8389 (2007)

175. Sukumaran, S.K., Grest, G.S., Kremer, K., Everaers, R.: J. Polym. Sci., Part B, Polym. Phys. 43, 917 (2005)

176. Kröger, M.: Comput. Phys. Commun. 168, 209 (2005)

177. Shanbhag, S., Kröger, M.: Macromolecules 40, 2897 (2007)

178. Tzoumanekas, C., Theodorou, D.N.: Macromolecules 39, 4592 (2006)

179. Tzoumanekas, C., Theodorou, D.N.: Curr. Opin. Solid State Mater. Sci. 10, 61 (2006)

180. Hoy, R.S., Foteinopoulou, K., Kröger, M.: Phys. Rev. E 80 (2009)

181. Gido, S.P., Gunther, J., Thomas, E.L., Hoffman, D.: Macromolecules 26, 4506 (1993)

182. Gido, S.P., Thomas, E.L.: Macromolecules 27, 849 (1994)

183. Netz, R., Andelman, D., Schick, M.: Phys. Rev. Lett. 79, 1058 (1997)

184. Matsen, M.W.: J. Chem. Phys. 107, 8110 (1997)

185. Duque, D., Schick, M.: J. Chem. Phys. 113, 5525 (2000)

186. Duque, D., Katsov, K., Schick, M.: J. Chem. Phys. 117, 10315 (2002)

187. Alder, B.J., Wainwright, T.E.: J. Chem. Phys. 27, 1208 (1957)

188. Wilding, N.B., Bruce, A.D.: Phys. Rev. Lett. 85, 5138 (2000)

189. Schilling, T., Schmid, F.: J. Chem. Phys. 131, 231102 (2009)

190. Hubbard, J.: Phys. Rev. Lett. 3, 77 (1959)

191. Matsen, M.W., Schick, M.: Phys. Rev. Lett. 72, 2660 (1994)

192. Alexander-Katz, A., Moreira, A.G., Sides, S.W., Fredrickson, G.H.: J. Chem. Phys. 122, 014904 (2005)

193. Ganesan, V., Fredrickson, G.H.: Europhys. Lett. 55, 814 (2001)

194. Reister, E., Müller, M., Binder, K.: Phys. Rev. E 64, 041804 (2001)

195. Fredrickson, G.H., Ganesan, V., Drolet, F.: Macromolecules 35, 16 (2002)

196. MacDowell, L.G., Virnau, P., Müller, M., Binder, K.: J. Chem. Phys. 120, 5293 (2004)

197. Cheng, X.Y., Lin, L., E, W.N., Zhang, P.W., Shi, A.C.: Phys. Rev. Lett. 104, 148301 (2010)

198. Sheu, S.Y., Mou, C.Y., Lovett, R.: Phys. Rev. E 51, R3795 (1995)

199. Grochola, G.: J. Chem. Phys. 120, 2122 (2004)

200. Hukushima, K., Nemoto, K.: J. Phys. Soc. Jpn. 65, 1604 (1996)

201. Hansmann, U.H.E.: Chem. Phys. Lett. 281, 140 (1997)

202. Sugita, Y., Okamoto, Y.: Chem. Phys. Lett. 314, 141 (1999) 
203. Lyubartsev, A.P., Martsinovski, A.A., Shevkunov, S.V., Vorontsov-Velyaminov, P.N.: J. Chem. Phys. 96, 1776 (1992)

204. Wang, F.G., Landau, D.P.: Phys. Rev. Lett. 86, 2050 (2001)

205. Virnau, P., Müller, M.: J. Chem. Phys. 120, 10925 (2004)

206. Widom, B.: J. Chem. Phys. 39, 2808 (1963)

207. Martinez-Veracoechea, F.J., Escobedo, F.A.: Macromolecules 40, 7354 (2007)

208. Frenkel, D., Smit, B.: Understanding Molecular Simulation. Academic Press, London (2002)

209. Siepmann, J.I.: Mol. Phys. 70, 1145 (1990)

210. Siepmann, J.I., Frenkel, D.: Mol. Phys. 75, 59 (1992)

211. de Pablo, J.J., Laso, M., Suter, U.W.: J. Chem. Phys. 97, 2817 (1992)

212. Shing, K.S., Gubbins, K.E.: Mol. Phys. 46, 1109 (1982)

213. Daoulas, K.C., Cavallo, A., Shenhar, R., Müller, M.: Soft Matter 5, 4499 (2009)

214. Daoulas, K.C., Cavallo, A., Shenhar, R., Müller, M.: Phys. Rev. Lett. 105, 108301 (2010)

215. Bates, F.S., Fredrickson, G.H.: Phys. Today 52, 32 (1999)

216. Fredrickson, G.H.: J. Chem. Phys. 117, 6810 (2002)

217. Gallavotti, G., Verboven, E.: Nuovo Cimento B-Gen. Phys. 28, 274 (1975)

218. Lovett, R., Buff, F.P.: Physica A 172, 147 (1991) 